\title{
About Classical to Quantum Weyl Correspondence
}

\author{
Alfred Wünsche \\ Institute of Physics, Humboldt University, Berlin, Germany \\ Email: alfred.wuensche@physik.hu-berlin.de
}

How to cite this paper: Wünsche, A. (2017) About Classical to Quantum Weyl Correspondence. Advances in Pure Mathematics, 7, 533-582.

https://doi.org/10.4236/apm.2017.710034

Received: September 14, 2017

Accepted: October 27, 2017

Published: October 30, 2017

Copyright $\odot 2017$ by author and Scientific Research Publishing Inc.

This work is licensed under the Creative Commons Attribution International License (CC BY 4.0).

http://creativecommons.org/licenses/by/4.0/

\begin{abstract}
After developing the mathematical means for the correspondence of classical phase-space function to quantum-mechanical operators with symmetrical ordering of the basic canonical operators in the sense of Weyl the approach is applied to an infinite series of classical monomial functions of the canonical variables. These include as well as pure powers of the amplitude $|\alpha|^{k},(k=0,1,2, \cdots)$ as also basic periodic functions $\mathrm{e}^{\mathrm{i} l \varphi},(l= \pm 1, \pm 2, \cdots)$ of the phase $\varphi$ with their quantum-mechanical correspondence. In the representation by number states $|n\rangle,(n=0,1, \cdots)$, all the considered operators involve the Jacobi polynomials as the essential formative element. Whereas the quantity $\overline{\mathcal{N}\left\{a^{\dagger 2} a^{2}\right\}}-{\overline{\mathcal{N}}\left\{a^{\dagger} a\right\}}^{2}$ in normal ordering due to its indeterminacy leads to the introduction of the notions of sub- and super-Poissonian statistics the analogous quantity in (Weyl) symmetrical ordering $\overline{\mathcal{S}\left\{a^{\dagger 2} a^{2}\right\}}-{\overline{\mathcal{S}}\left\{a^{\dagger} a\right\}}^{2}$ is positive definite and satisfies an inequality. The notions of sub- and super-Poissonian statistics are problematic when they are used for the definition of nonclassicality of states since the mentioned measure in normal ordering does not determine the Poisson statistics in their middle in unique way but determines only a large set of statistics which may be very far in the sense of the Hilbert-Schmidt distance from a Poisson statistics that is discussed.
\end{abstract}

\section{Keywords}

Wigner Quasiprobability, Symmetrical (Weyl) Ordering,

Nonclassicality of Steates, Distance of States, Sub- and Super-Poissonian

Statistics, Phase Operator, Laguerre 2D Polynomials, Jacobi Polynomials

\section{Introduction}

The basic part of quantum mechanics originated from classical Hamilton 
mechanics and was developed in the twenties and thirties of last century. The canonical variables $(q, p)$ of the phase space in the Hamilton function $H(q, p)$ were substituted by operators $(Q, P)$ which obey the commutation relations $[Q, P]=\mathrm{i} \hbar I,(\hbar$ Planck's action quantum $h$ divided by $2 \pi$ and $I$ identity operator of the Hilbert space of the representation of the operator algebra). In general, a classical function $A(q, p)$ of the canonical variables $(q, p)$ cannot be translated into a corresponding quantum-mechanical function $A(Q, P)$ without additional rules for operator ordering since $Q P \neq P Q$. Luckily, this did not play a role in the first very successful applications of the Schrödinger equation with a Hamilton operator $H$ with additively separated classical kinetic energy $T(\boldsymbol{p}) \rightarrow T(\boldsymbol{P})$ from potential energy $U(\boldsymbol{q}) \rightarrow U(\boldsymbol{Q})$ with $\boldsymbol{q} \equiv \boldsymbol{r}$ the position vector and $p$ the (canonical) momentum vector. Thus it was translated into the quantum-mechanical Hamilton operator $H=T(\boldsymbol{P})+U(\boldsymbol{Q})$ where $(\boldsymbol{q}, \boldsymbol{p})$ in classical mechanics are, in general, three-dimensional vectors and $(\boldsymbol{Q}, \boldsymbol{P})$ corresponding vector operators with independent components and independent commutation relations $\left[Q_{j}, P_{k}\right]=\mathrm{i} \hbar \delta_{j, k}$. Luckily also, this did not play a role in the translation of the classical angular momentum $\mathbf{L}(\boldsymbol{q}, \boldsymbol{p}) \equiv[\boldsymbol{q}, \boldsymbol{p}]$ (vector product of $\boldsymbol{q}$ with $\boldsymbol{p}$ ) or $L_{i}(\boldsymbol{q}, \boldsymbol{p}) \equiv \varepsilon_{i j k} q_{j} p_{k}$ into a quantum-mechanical operator since there are only combined independent (commutating) components of $Q_{j}$ and $P_{k},(k \neq j)$, in the quantum-mechanical operator $L_{i}=\varepsilon_{i j k} Q_{j} P_{k}$. In quantum field theories such as quantum electrodynamics and optics where one usually speaks about independent modes the canonical variables $(q, p) \rightarrow(Q, P)$ are internal field variables whereas the position $r$ and the time $t$ are classical variables as parameters of each mode determining the shape of the field and $p$ is not the momentum of the field, for example, of a wave packet or beam ${ }^{1}$. In present paper, however, we mainly use the transition from canonical coordinates $(q, p)$ to complex coordinates $\left(\alpha, \alpha^{*}\right)$ and from canonical operators $(Q, P)$ to annihilation and creation operators $\left(a, a^{\dagger}\right)$, correspondingly.

Hermann Weyl in [1] and in his book [2] from 1928 (chap. IV, §14) proposed a general rule for the translation of arbitrary functions $A(q, p)$ of the canonical phase-space variables in a unique way into quantum-mechanical operator-ordered functions $A(Q, P)$ of the operators $(Q, P)$. His way was via the Fourier transform of the classical function $A(q, p)$ (denoted there in the way $f(p, q)=\iint_{-\infty}^{+\infty} e(\sigma p+\tau q) \xi(\sigma, \tau) \mathrm{d} \sigma \mathrm{d} \tau$ where $e(x) \equiv \mathrm{e}^{\mathrm{i} x}$ and $\xi(\sigma, \tau)$ is the Fourier transform of $f(p, q)$ and $(p, q)$ are here operators). On the opposite our preliminarily written "function" $A(Q, P)$ is not well and uniquely defined without an ordering rule. The form proposed by Weyl is called the (Weyl) symmetrical ordering and we denote it by $\mathcal{S}\{A(Q, P)\}$. In this symmetrical ordering of the operators $(Q, P)$ in a function $A(Q, P)$ we may

${ }^{1}$ Therefore, in quantum optics it is usually unfavorable to denote the canonical variables of the phase space by $(x, p)$ that may lead to confusion with spatial variables. Energy and momentum of a light-wave packet are connected with quadratic combinations of the canonical variables $\boldsymbol{q}$ and $\boldsymbol{p}$ involving frequency and wave vector as parameters. 
consider $(Q, P)$ first as the classical variables $(q, p)$ with respect, for example, of Taylor series expansions and may order then their sum terms in corresponding way but we can make this also in the form of the Fourier integral as a whole. Our way of definition of symmetrical ordering (in the sense of Weyl) in the following is fully equivalent to that of Weyl but looks only a little different at the first glance. We explain this later in detail. To a classical function $A(q, p)$ over the phase space corresponds then a uniquely defined operator $A \equiv \mathcal{S}\{A(Q, P)\}$. The calculation of expectation values of symmetrically ordered operators is best suited to the Wigner function $W(q, p)$ which is a quasiprobability over the phase space introduced in 1932 by Eugen P. Wigner [3] (republished in [4]). Together with the Weyl ordering this is often called the Weyl-Wigner formalism of correspondence between classical and quantum mechanics [5]. Other correspondences of classical phase-space functions to quantum-mechanical operators are possible, in particular, the normally-ordered correspondence for which another quasiprobability $P(q, p)$ called the Glauber-Sudarshan quasiprobability [6] [7] [8] [9] is best suited for the calculation of expectation values of such operators if we know only the corresponding classical phase-space function. For anti-normally-ordered operators, the Husimi-Kano quasiprobability $Q(q, p)$ takes on this place. Other quasiprobabilities for the calculation of the expectation values of arbitrarily ordered operators are also appropriate, however with more complicated formulae in this case.

The symmetrical ordering in the sense of Weyl possesses the highest degeneracy of the operator kernel in the integral transform defining it and every change of this kernel removes this degeneracy in different possible directions [10]. From the theoretical point of view the symmetrical ordering is the most aesthetical and attractive one but does nature also prefer it? The zero-point energy of the modes in quantum optics and its consequences, for example, in the theoretically derived and experimentally observed (?) Casimir effect gives some evidence that the symmetrical ordering of operators is, at least, in quantum optics likely the correct correspondence between classical and quantum physics. An early and well organized representation of many problems concerning the different quasiprobabilities and ordering used in quantum optics is given by Perina [11]. Problems of the determination of a phase operator in quantum optics are discussed and referred in detail by Peřinová, Lukš and Peřina [12].

There are some technical difficulties to implement the explicit calculation of the symmetrically ordered operators corresponding to given classical phase-space functions in general cases, in particular, in the Fock or number state representation. A basic result for operators of the form

$\mathcal{S}\left\{\left(\sqrt{a a^{\dagger}}\right)^{k}\left(\sqrt{\frac{a}{a^{\dagger}}}\right)^{l}\right\} \equiv \mathcal{S}\left\{a^{m} a^{\dagger n}\right\}$ for integers $\quad(k, l), \quad(k=m+n, l=m-n)$, was communicated in [13] with some promise to give its detailed derivation in another paper. We discuss this in present paper but the more technical details of this calculation we shift to the Appendices. We connect the results with other 
already known more special results. In particular, we consider from Section 6 on a set of classical basic functions $A^{(k, l)}\left(\alpha, \alpha^{*}\right)$ and determine the corresponding basic operators $A^{(k, l)}$ in number representation using the Jacobi polynomials for the representation of the coefficients. In Section 8 we generalize this to smoothing of the operators by means of normalized Gaussian bell functions. The results for the special case $A^{(k, 0)}$ can be also obtained by integration of the general Wigner quasiprobability over the angle and the special case $A^{(0, l)}$ by integration of the Wigner quasiprobability over the radius that for this last case was first made by Garraway and Knight [14] (see also [12]). One has to liberate oneself in these cases from the general density operator $\rho$ in the Wigner quasiprobability $W\left(\alpha, \alpha^{*}\right)$ in complex representation and obtain then in last case the quantum equivalents $A^{(0, l)}$ to the basic classical periodic phase functions $\mathrm{e}^{\mathrm{i} l \varphi},(l=0, \pm 1, \pm 2, \cdots)$.

In Section 12 we derive the connection of the symmetrically ordered operators $\mathcal{S}\left\{a^{\dagger k} a^{k}\right\}$ to powers of the number operator $N \equiv a^{\dagger} a$. This suggests to use the expectation values (notation by overlining operators) $\overline{\mathcal{S}\left\{a^{\dagger 2} a^{2}\right\}}-{\overline{\mathcal{S}}\left\{a^{\dagger} a\right\}}^{2}$ which are positive definite as alternative to the corresponding normally ordered quantity $\overline{\mathcal{N}\left\{a^{\dagger 2} a^{2}\right\}}-\overline{\mathcal{N}\left\{a^{\dagger} a\right\}}{ }^{2}$ which is indefinite and leads in dependence of its negativity or positivity to the definition of sub- and super-Poissonian quantum statistics which are problematic when they are used for the definition of non-classicality of states.

\section{Basic Notions and Displacement Operator}

Note: The trace of an operator $A$ is denoted by $\langle A\rangle$ and the expectation value of $A$ by overlining the operator $\bar{A} \equiv\langle\varrho A\rangle$ if $\varrho$ is the density operator.

In this Section, we prepare the description of the symmetrical (Weyl) correspondence of classical to quantum mechanics by some, in principle known, basic notions and explain our notations. We consider a Hamilton system of one degree of freedom in canonical variables $(q, p)$. Additionally, we introduce complex variables $\left(\alpha, \alpha^{*}\right)$ in the following way

$$
\begin{aligned}
\left(\alpha, \alpha^{*}\right) & =\frac{1}{\sqrt{2 \hbar}}(q+\mathrm{i} p, q-\mathrm{i} p), \Leftrightarrow(q, p)=\sqrt{2 \hbar}\left(\frac{\alpha+\alpha^{*}}{2},-\mathrm{i} \frac{\alpha-\alpha^{*}}{2}\right) \\
\left(\frac{\partial}{\partial \alpha}, \frac{\partial}{\partial \alpha^{*}}\right) & =\sqrt{2 \hbar}\left(\frac{1}{2}\left(\frac{\partial}{\partial q}-\mathrm{i} \frac{\partial}{\partial p}\right), \frac{1}{2}\left(\frac{\partial}{\partial q}+\mathrm{i} \frac{\partial}{\partial p}\right)\right), \quad \frac{\mathrm{i}}{2} \mathrm{~d} \alpha \wedge \mathrm{d} \alpha^{*}=\frac{\mathrm{d} q \wedge \mathrm{d} p}{2 \hbar}
\end{aligned}
$$

with correspondence to the basic quantum-mechanical operators $(q, p) \Leftrightarrow(Q, P)$ and their combinations $\left(\alpha, \alpha^{*}\right) \Leftrightarrow\left(a, a^{\dagger}\right)$ which become the annihilation $a$ and creation $a^{\dagger}$ operator for a harmonic oscillator

$$
\left(a, a^{\dagger}\right)=\frac{1}{\sqrt{2 \hbar}}(Q+\mathrm{i} P, Q-\mathrm{i} P) \Leftrightarrow(Q, P)=\sqrt{2 \hbar}\left(\frac{a+a^{\dagger}}{2},-\mathrm{i} \frac{a-a^{\dagger}}{2}\right)
$$

They obey the commutation relations ( $I$ identity operator of the representation space (Hilbert space)) 


$$
[Q, P]=\mathrm{i} \hbar I, \quad\left[a, a^{\dagger}\right]=I
$$

The reason that we introduce the complex substitutes of the canonical variables by (2.1) and not simply by $\left(z, z^{*}\right)=(q+\mathrm{i} p, q-\mathrm{i} p)$ is that we are interested in the following mainly in the expectation values of ordered functions of $\left(a, a^{\dagger}\right)$ which are (boson) annihilation and creation operators for harmonic oscillators of modes of the electromagnetic field. However, in transitions from quantum to classical mechanics and optics setting $\hbar=0$ one has to be cautious using the operators $\left(a, a^{\dagger}\right)$. For example, in the operator $N \equiv a^{\dagger} a=\frac{Q^{2}+P^{2}}{2 \hbar}-\frac{1}{2} I$ one cannot set $\hbar=0$ since $N$ (number operator) does not possess a classical analogue and only after multiplication of this operator with $\hbar$ we get an operator with a classical analogue in form of the function $\frac{q^{2}+p^{2}}{2}=\hbar \alpha \alpha^{*}$ proportional to the intensity or to energy and momentum of the field.

Starting from the well-defined operator function $\exp \left(\mu a+v a^{\dagger}\right)$ with arbitrary parameters $(\mu, v)$ the symmetrical Weyl ordering can be defined by (e.g., [15])

$$
\begin{aligned}
\exp \left(\mu a+v a^{\dagger}\right)= & \sum_{j=0}^{\infty} \frac{\left(\mu a+v a^{\dagger}\right)^{j}}{j !} \equiv \sum_{k=0}^{\infty} \sum_{l=0}^{\infty} \frac{\mu^{k} v^{l}}{k ! l !} \mathcal{S}\left\{a^{k} a^{\dagger l}\right\} \equiv \mathcal{S}\left\{\exp \left(\mu a+v a^{\dagger}\right)\right\} \\
& \left(\mu a+v a^{\dagger}\right)^{j}=\sum_{k=0}^{j} \frac{j !}{k !(j-k) !} \mu^{k} v^{j-k} \mathcal{S}\left\{a^{k} a^{\dagger j-k}\right\}
\end{aligned}
$$

From this follows for integer $(k, l)$

$$
\mathcal{S}\left\{a^{k} a^{\dagger l}\right\}=\left\{\frac{\partial^{k+l}}{\partial \mu^{k} \partial v^{l}} \exp \left(\mu a+v a^{\dagger}\right)\right\}_{\mu=v=0}
$$

or using the binomial formula for $\left(\mu a+v a^{\dagger}\right)^{k+l}$ with observation of the non-commutativity of $a$ and $a^{\dagger}$

$$
\mathcal{S}\left\{a^{k} a^{\dagger l}\right\}=\frac{1}{(k+l) !} \frac{\partial^{k+l}}{\partial \mu^{k} v^{l}}\left(\mu a+v a^{\dagger}\right)^{k+l}
$$

The symbol $\mathcal{S}\{\ldots\}$ is not a linear operator since ${ }^{2}$

$$
\mathcal{S}\left\{a^{k} a^{\dagger l}\right\}=\mathcal{S}\left\{a^{\dagger l} a^{k}\right\}=\mathcal{S}\left\{\mathcal{P}\left(a^{k} a^{\dagger l}\right)\right\}
$$

where $\mathcal{P}\left(a^{k} a^{\dagger l}\right)$ means here the product of $k$ operators $a$ and $l$ operators $a^{\dagger}$ in arbitrary order (i.e., permutations) but linear combinations of them are understood in the sense of the distributive law

$$
\mathcal{S}\left\{\kappa A\left(a, a^{\dagger}\right)+\lambda B\left(a, a^{\dagger}\right)\right\}=\kappa \mathcal{S}\left\{A\left(a, a^{\dagger}\right)\right\}+\lambda \mathcal{S}\left\{B\left(a, a^{\dagger}\right)\right\}
$$

This gives the possibility to determine linear spaces of ordered operators if one introduces a system of basis operators. Clearly, two operators $\mathcal{S}\left\{A\left(a, a^{\dagger}\right)\right\}$ and $\mathcal{S}\left\{B\left(a, a^{\dagger}\right)\right\}$ are, in general, noncommutative.

${ }^{2}$ Some authors write the corresponding classical variables within an ordering symbol (here symbol $\mathcal{S}\{\ldots\})$ that means, e.g., $\mathcal{S}\left\{A\left(\alpha, \alpha^{*}\right)\right\}$ instead of our $\mathcal{S}\left\{A\left(a, a^{\dagger}\right)\right\}$. 
In the following we use the displacement operator $D\left(\alpha, \alpha^{*}\right)$ defined by (e.g., [7] [8] [11])

$$
\begin{aligned}
D\left(\alpha, \alpha^{*}\right) & =\exp \left(\alpha a^{\dagger}-\alpha^{*} a\right) \equiv \mathcal{S}\left\{\exp \left(\alpha a^{\dagger}-\alpha^{*} a\right)\right\} \\
& =\exp \left(-\frac{\alpha \alpha^{*}}{2}\right) \exp \left(\alpha a^{\dagger}\right) \exp \left(-\alpha^{*} a\right) \\
& \equiv \exp \left(-\frac{\alpha \alpha^{*}}{2}\right) \mathcal{N}\left\{\exp \left(\alpha a^{\dagger}-\alpha^{*} a\right)\right\}
\end{aligned}
$$

where the symbol $\mathcal{N}\{\ldots\}$ means normal ordering of the content in braces (all powers of $a^{\dagger}$ in front of powers of $a$ ). From (2.9) follows for its Hermitean adjoint operator

$$
\left(D\left(\alpha, \alpha^{*}\right)\right)^{\dagger}=\exp \left(\alpha^{*} a-\alpha a^{\dagger}\right)=\left(D\left(\alpha, \alpha^{*}\right)\right)^{-1}=D\left(-\alpha,-\alpha^{*}\right)
$$

To obtain the normally ordered form of the displacement operator on the right-hand side of (2.9) we applied here the well-known theorem (e.g., [7])

$$
\exp (A) \exp (B)=\exp \left(A+B+\frac{1}{2}[A, B]\right), \quad \text { if }[A,[A, B]]=[B,[A, B]]=0
$$

which is true for arbitrary operators $A$ and $B$ which commute with their commutator $[A, B]$. This is a special case of the general

Baker-Campbell-Hausdorff-Dynkin formula for the product of the exponentials of two operators which for the case in (2.11) can be proved more directly, e.g., [7]. In the following we apply this theorem repeatedly.

The displacement operator possesses the property

$$
D\left(\alpha, \alpha^{*}\right) a\left(D\left(\alpha, \alpha^{*}\right)\right)^{\dagger}=a-\alpha I, \quad D\left(\alpha, \alpha^{*}\right) a^{\dagger}\left(D\left(\alpha, \alpha^{*}\right)\right)^{\dagger}=a^{\dagger}-\alpha^{*} I
$$

For the product of two displacement operators one finds applying (2.11)

$$
D\left(\alpha, \alpha^{*}\right) D\left(\beta, \beta^{*}\right)=\exp \left(\frac{1}{2}\left(\alpha \beta^{*}-\alpha^{*} \beta\right)\right) D\left(\alpha+\beta, \alpha^{*}+\beta^{*}\right)
$$

Furthermore, we need the normal ordering of operators of the form $\exp \left(\kappa a^{\dagger} a\right)$ with parameter $\kappa$. The following relation is well known, e.g., [16] (chap. 3.3., pp. 156/157) ${ }^{3}$

$$
\exp (\kappa N)=\exp \left(\kappa a^{\dagger} a\right)=\sum_{k=0}^{\infty} \frac{\left(\mathrm{e}^{\kappa}-1\right)^{k}}{k !} a^{\dagger k} a^{k} \equiv \mathcal{N}\left\{\exp \left(\left(\mathrm{e}^{\kappa}-1\right) a^{\dagger} a\right)\right\}
$$

${ }^{3}$ Louisell gives two proofs, the first using the diagonal matrix elements with coherent states and the second by a differential equation. One may add a further easy one using the matrix elements in the basis of number states $|n\rangle,(n=0,1, \cdots)$ as the eigenstates of the number operator $N \equiv a^{\dagger} a$, with $N|n\rangle=n|n\rangle, \quad a|n\rangle=\sqrt{n}|n-1\rangle, a^{\dagger}|n\rangle=\sqrt{n+1}|n+1\rangle$ and the completeness relation $\sum_{n=0}^{\infty}|n\rangle\langle n|=I$ $\mathcal{N}\left\{\exp \left(\left(\mathrm{e}^{\kappa}-1\right) a^{\dagger} a\right)\right\} \equiv \sum_{k=0}^{\infty} \frac{\left(\mathrm{e}^{\kappa}-1\right)^{k}}{k !} a^{\dagger k}\left(\sum_{n=0}^{\infty}|n\rangle\langle n|\right) a^{k}=\sum_{k=0}^{\infty} \frac{\left(\mathrm{e}^{\kappa}-1\right)^{k}}{k !} \sum_{n=0}^{\infty}\left(\sqrt{\frac{(n+k) !}{n !}}\right)^{2}|n+k\rangle\langle n+k|$. $=\sum_{n^{\prime}=0}^{\infty}\left(\sum_{k=0}^{n^{\prime}} \frac{n^{\prime} !}{k !\left(n^{\prime}-k\right) !}\left(\mathrm{e}^{\kappa}-1\right)^{k}\right)\left|n^{\prime}\right\rangle\left\langle n^{\prime}\left|=\sum_{n=0}^{\infty}\left(\mathrm{e}^{\kappa}-1+1\right)^{n}\right| n\right\rangle\langle n|=\mathrm{e}^{\kappa N}$ 
The conversion of (2.14) with respect to the parameter $\kappa$ as it is easily seen is

$$
\mathcal{N}\left\{\exp \left(\lambda a^{\dagger} a\right)\right\} \equiv \sum_{k=0}^{\infty} \frac{\lambda^{k}}{k !} a^{\dagger k} a^{k}=(1+\lambda)^{a^{\dagger} a}=(1+\lambda)^{N}
$$

For $\lambda=1,0,-1,-2$ one obtains the operators

$$
\begin{gathered}
\mathcal{N}\left\{\exp \left(a^{\dagger} a\right)\right\}=2^{N}, \quad \mathcal{N}\left\{\exp \left(0 a^{\dagger} a\right)\right\}=\mathcal{N}\{I\}=I \\
\mathcal{N}\left\{\exp \left(-a^{\dagger} a\right)\right\}=\lim _{\varepsilon \rightarrow 0} \varepsilon^{N}=|0\rangle\langle 0|, \quad \mathcal{N}\left\{\exp \left(-2 a^{\dagger} a\right)\right\}=(-1)^{N}=\Pi
\end{gathered}
$$

All these special operators play a certain role in quantum optics, in particular, $\mathcal{N}\left\{\exp \left(0 a^{\dagger} a\right)\right\}$ is the unity operator $I, \mathcal{N}\left\{\exp \left(-a^{\dagger} a\right)\right\}$ is the vacuum-state operator $|0\rangle\langle 0|$ and $\mathcal{N}\left\{\exp \left(-2 a^{\dagger} a\right)\right\}$ the parity operator $\Pi$ which we consider more in detail in next Section.

\section{Basic Relations of the Weyl Formalism and the Parity Operator}

The general formula for the transition from arbitrary classical phase-space functions $A(q, p)$ in the symmetrical Weyl ordering to quantum-mechanical operators $A \equiv \mathcal{S}\{A(Q, P)\}$ can be written

$$
A(q, p) \rightarrow A=\int \mathrm{d} q \wedge \mathrm{d} p A(q, p) \exp \left(-Q \frac{\partial}{\partial q}-P \frac{\partial}{\partial p}\right) \delta(q) \delta(p)
$$

or in complex representation by complex variables $\left(\alpha, \alpha^{*}\right)$ (see (2.1)) as follows (we do not use a new function symbol and set $A(q, p) \equiv A\left(\alpha, \alpha^{*}\right)$ )

$$
\begin{aligned}
A\left(\alpha, \alpha^{*}\right) \rightarrow A & =\int \frac{\mathrm{i}}{2} \mathrm{~d} \alpha \wedge \mathrm{d} \alpha^{*} A\left(\alpha, \alpha^{*}\right) \exp \left(-a \frac{\partial}{\partial \alpha}-a^{\dagger} \frac{\partial}{\partial \alpha^{*}}\right) \delta\left(\alpha, \alpha^{*}\right) \\
& =\int \frac{\mathrm{i}}{2} \mathrm{~d} \alpha \wedge \mathrm{d} \alpha^{*} A\left(\alpha, \alpha^{*}\right) \mathcal{S}\left\{\delta\left(\alpha I-a, \alpha^{*} I-a^{\dagger}\right)\right\} \\
& \equiv \mathcal{S}\left\{A\left(a, a^{\dagger}\right)\right\}
\end{aligned}
$$

where $\delta\left(\alpha, \alpha^{*}\right)$ is the two-dimensional delta function in complex representation according to

$$
\int \frac{\mathrm{i}}{2} \mathrm{~d} \alpha \wedge \mathrm{d} \alpha^{*} \delta\left(\alpha, \alpha^{*}\right) f\left(\alpha, \alpha^{*}\right)=f(0,0), \quad \delta\left(\alpha, \alpha^{*}\right)=2 \hbar \delta(q) \delta(p)
$$

The integration goes in both representations over the whole phase-plane in real or complex coordinates $(q, p) \leftrightarrow\left(\alpha, \alpha^{*}\right)$. After partial integration in the first line we find equivalently to (3.2)

$$
\begin{aligned}
A & =\int \frac{\mathrm{i}}{2} \mathrm{~d} \alpha \wedge \mathrm{d} \alpha^{*} \delta\left(\alpha, \alpha^{*}\right) \exp \left(a \frac{\partial}{\partial \alpha}+a^{\dagger} \frac{\partial}{\partial \alpha^{*}}\right) A\left(\alpha, \alpha^{*}\right) \\
& =\sum_{k=0}^{\infty} \sum_{l=0}^{\infty} \frac{\mathcal{S}\left\{a^{k} a^{\dagger l}\right\}}{k ! l !}\left\{\frac{\partial^{k+l}}{\partial \alpha^{k} \partial \alpha^{* l}} A\left(\alpha, \alpha^{*}\right)\right\}_{\alpha=\alpha^{*}=0}
\end{aligned}
$$

For the trace $\langle A\rangle$ of the operator $A$ we find from (3.2) or (3.7) 


$$
\langle A\rangle=\frac{1}{\pi} \int \frac{\mathrm{i}}{2} \mathrm{~d} \alpha \wedge \mathrm{d} \alpha^{*} A\left(\alpha, \alpha^{*}\right), \quad(\langle A\rangle \equiv \operatorname{Trace}(A))
$$

The factor $\frac{1}{\pi}$ in (3.5) in front of the integral $\int \frac{\mathrm{i}}{2} \mathrm{~d} \alpha \wedge \mathrm{d} \alpha^{*} A\left(\alpha, \alpha^{*}\right)$ is already an indication that non-orthogonal ("overlapping") states are involved in the definitions (these are the displaced number states; see below). The form of the classical-quantum correspondence in the second line of (3.2) is very near to the form given by Weyl if we make in addition the Fourier transformation of $\mathcal{S}\left\{\delta\left(\alpha I-a, \alpha^{*} I-a^{\dagger}\right)\right\}$ but our form has the advantage that we do not have to discuss the exact form of this transform (i.e., coefficient in front and factors in the exponent).

The inversion of (3.1) is (we denote the trace of an arbitrary operator $B$ by $\langle B\rangle)$

$$
A(q, p)=2 \pi \hbar\left\langle A \exp \left(-Q \frac{\partial}{\partial q}-P \frac{\partial}{\partial p}\right)\right\rangle \delta(q) \delta(p)
$$

or of the complex form (3.2)

$$
A\left(\alpha, \alpha^{*}\right)=\pi\left\langle A \exp \left(-a \frac{\partial}{\partial \alpha}-a^{\dagger} \frac{\partial}{\partial \alpha^{*}}\right)\right\rangle \delta\left(\alpha, \alpha^{*}\right)
$$

The transformation $A\left(\alpha, \alpha^{*}\right) \rightarrow A$ in (3.2) together with the inverse transformation $A \rightarrow A\left(\alpha, \alpha^{*}\right)$ in (3.7) is a mapping which preserves the distribution law for arbitrary complex numbers $\mu$ and $v$

$$
A\left(\alpha, \alpha^{*}\right) \leftrightarrow A, \quad B\left(\alpha, \alpha^{*}\right) \leftrightarrow B \Leftrightarrow \mu A\left(\alpha, \alpha^{*}\right)+v B\left(\alpha, \alpha^{*}\right) \leftrightarrow \mu A+v B
$$

due to the linearity of the transformation.

We now make the normal ordering of the operator which plays a role in (3.2) in the transition from classical phase-space functions $A\left(\alpha, \alpha^{*}\right)$ to quantummechanical operators $A$ which are symmetrically ordered equivalents in the sense of Weyl

$$
\begin{aligned}
& \exp \left(-a \frac{\partial}{\partial \alpha}-a^{\dagger} \frac{\partial}{\partial \alpha^{*}}\right) \delta\left(\alpha, \alpha^{*}\right) \\
& =\exp \left(-a^{\dagger} \frac{\partial}{\partial \alpha^{*}}\right) \exp \left(-a \frac{\partial}{\partial \alpha}\right) \exp \left(\frac{1}{2} \frac{\partial^{2}}{\partial \alpha \partial \alpha^{*}}\right) \delta\left(\alpha, \alpha^{*}\right) \\
& =\exp \left(-a^{\dagger} \frac{\partial}{\partial \alpha^{*}}\right) \exp \left(-a \frac{\partial}{\partial \alpha}\right) \frac{2}{\pi} \exp \left(-2 \alpha \alpha^{*}\right) \\
& =\frac{2}{\pi} \mathcal{N}\left\{\exp \left(-2(a-\alpha I)\left(a^{\dagger}-\alpha^{*} I\right)\right)\right\} \\
& =\frac{2}{\pi} D\left(\alpha, \alpha^{*}\right) \mathcal{N}\left\{\exp \left(-2 a a^{\dagger}\right)\right\}\left(D\left(\alpha, \alpha^{*}\right)\right)^{\dagger} \\
& =\frac{2}{\pi} D\left(\alpha, \alpha^{*}\right)(-1)^{a^{\dagger} a}\left(D\left(\alpha, \alpha^{*}\right)\right)^{\dagger}
\end{aligned}
$$

In the derivation we used the identity

$$
\exp \left(\frac{r}{2} \frac{\partial^{2}}{\partial z \partial z^{*}}\right) \delta\left(z, z^{*}\right)=\frac{2}{\pi r} \exp \left(-\frac{2 z z^{*}}{r}\right)
$$


specialized to $r=1$, which generally can be proved by two-dimensional Fourier transformation. The operator $\exp \left(\frac{r}{2} \frac{\partial^{2}}{\partial z \partial z^{*}}\right)$ with positive values of the parameter $r$ applied to a function $f\left(z, z^{*}\right)$ makes a smoothing of this function.

The operator $\Pi$ defined by (see also (2.15) and (2.16) for the different representations)

$$
\Pi \equiv(-1)^{a^{\dagger} a}=(-1)^{N}=\exp ( \pm \mathrm{i} \pi N)=\mathcal{N}\left\{\exp \left(-2 a a^{\dagger}\right)\right\}=\sum_{k=0}^{\infty} \frac{(-2)^{k}}{k !} a^{\dagger k} a^{k}
$$

is called the parity operator and the operator $\Pi\left(\alpha, \alpha^{*}\right)$ defined by

$$
\Pi\left(\alpha, \alpha^{*}\right) \equiv D\left(\alpha, \alpha^{*}\right) \Pi\left(D\left(\alpha, \alpha^{*}\right)\right)^{\dagger}
$$

the displaced parity operator, correspondingly. Thus we obtained in (3.9) the basic relation

$$
\begin{aligned}
\frac{2}{\pi} \Pi\left(\alpha, \alpha^{*}\right) & =\exp \left(-a \frac{\partial}{\partial \alpha}-a^{\dagger} \frac{\partial}{\partial \alpha^{*}}\right) \delta\left(\alpha, \alpha^{*}\right) \\
& =\sum_{k=0}^{\infty} \sum_{l=0}^{\infty} \frac{(-1)^{k+l}}{k ! l !} \mathcal{S}\left\{a^{k} a^{\dagger l}\right\} \frac{\partial^{k+l}}{\partial \alpha^{k} \partial \alpha^{* l}} \delta\left(\alpha, \alpha^{*}\right)
\end{aligned}
$$

which plays a role in the following. We see here that the limiting transition $\left(\alpha, \alpha^{*}\right) \rightarrow(0,0)$ on the right-hand side in (3.13) is not possible in this way and the representation of the parity operator $\Pi=\Pi\left(\alpha \rightarrow 0, \alpha^{*} \rightarrow 0\right)$ is not locally possible and the representation of $\Pi$ by the symmetrically ordered operators $\mathcal{S}\left\{a^{k} a^{\dagger l}\right\}$ does not exist in contrast to the representation by the normally ordered operators $\mathcal{N}\left\{a^{k} a^{\dagger l}\right\}=a^{\dagger k} a^{l}$ (see (3.11)).

The parity operator $\Pi$ is a Hermitean and at once an idempotent operator (squared it is equal to the identity operator)

$$
\Pi=\Pi^{\dagger}=\Pi^{-1}, \quad \Pi^{2}=I,
$$

with the following interesting properties of transformation of $\left(a, a^{\dagger}\right)$ justifying its name

$$
\begin{aligned}
\Pi a \Pi^{\dagger} & =\mathrm{e}^{\mathrm{i} \pi N} a \mathrm{e}^{-\mathrm{i} \pi N}=a+\frac{\mathrm{i} \pi}{1 !} \underbrace{[N, a]}_{=-a}+\frac{(\mathrm{i} \pi)^{2}}{2 !} \underbrace{[N,[N, a]]}_{=+a}+\cdots=\left(\sum_{n=0}^{\infty} \frac{(-\mathrm{i} \pi)^{n}}{n !}\right) a \\
& =\mathrm{e}^{-\mathrm{i} \pi} a=-a, \quad \Pi a^{\dagger} \Pi^{\dagger}=-a^{\dagger} \Rightarrow \Pi D\left(\alpha, \alpha^{*}\right) \Pi^{\dagger}=D\left(-\alpha,-\alpha^{*}\right)
\end{aligned}
$$

From these commutation properties follows using (3.15)

$$
\begin{aligned}
\Pi\left(\alpha, \alpha^{*}\right) & =D\left(\alpha, \alpha^{*}\right) \Pi\left(D\left(\alpha, \alpha^{*}\right)\right)^{\dagger}=D\left(\alpha, \alpha^{*}\right) D\left(\alpha, \alpha^{*}\right) \Pi \\
& =D\left(2 \alpha, 2 \alpha^{*}\right) \Pi=\Pi D\left(-\alpha,-\alpha^{*}\right) D\left(-\alpha,-\alpha^{*}\right)=\Pi D\left(-2 \alpha,-2 \alpha^{*}\right)
\end{aligned}
$$

The parity operator $\Pi$ possesses only the two eigenvalues +1 and -1 to even and odd number states $|n\rangle$ and $\langle n|$ as right-hand and corresponding left-hand eigenstates

$$
(-1)^{N}|n\rangle=(-1)^{n}|n\rangle, \quad\langle n|(-1)^{N}=(-1)^{n}\langle n|, \quad\left(N \equiv a^{\dagger} a\right)
$$


that leads to the following possible representations by eigenstates

$$
\Pi=\sum_{n=0}^{\infty}(-1)^{n}|n\rangle\left\langle n\left|, \quad \Pi\left(\alpha, \alpha^{*}\right)=\sum_{n=0}^{\infty}(-1)^{n} D\left(\alpha, \alpha^{*}\right)\right| n\right\rangle\langle n|\left(D\left(\alpha, \alpha^{*}\right)\right)^{\dagger}
$$

It is a highly degenerate operator which therefore admits many other representations by linear combinations of the eigenstates to the eigenvalues +1 and -1 separately. The displaced parity operator $\Pi\left(\alpha, \alpha^{*}\right)$ defined in (3.12) possesses the same eigenvalues +1 and -1 but to displaced number states $|\alpha, n\rangle$ defined by

$$
|\alpha, n\rangle \equiv D\left(\alpha, \alpha^{*}\right)|n\rangle, \quad(n=0,1,2, \cdots)
$$

as right-hand eigenstates of $\Pi\left(\alpha, \alpha^{*}\right)$ according to

$$
\begin{aligned}
\Pi\left(\alpha, \alpha^{*}\right)|\alpha, n\rangle & =D\left(\alpha, \alpha^{*}\right) \Pi\left(D\left(\alpha, \alpha^{*}\right)\right)^{-1} D\left(\alpha, \alpha^{*}\right)|n\rangle \\
& =D\left(\alpha, \alpha^{*}\right) \Pi|n\rangle \equiv(-1)^{n} D\left(\alpha, \alpha^{*}\right)|n\rangle=(-1)^{n}|\alpha, n\rangle
\end{aligned}
$$

and similar for the left-hand eigenstates $\langle\alpha, n|$. The displaced number states $|\alpha, n\rangle$ are ortho-normalized for discrete $(m, n)$ and arbitrary fixed $\alpha$ according to

$$
\langle\alpha, m \mid \alpha, n\rangle=\left\langle m\left|\left(D\left(\alpha, \alpha^{*}\right)\right)^{\dagger} D\left(\alpha, \alpha^{*}\right)\right| n\right\rangle=\langle m \mid n\rangle=\delta_{m, n}
$$

and they obey the following relation (see below (3.25))

$$
\frac{1}{\pi} \int \frac{\mathrm{i}}{2} \mathrm{~d} \alpha \wedge \mathrm{d} \alpha^{*}|\alpha, n\rangle\langle\alpha, m|=\langle m \mid n\rangle I=\delta_{m, n} I
$$

This means that the states $|\alpha, n\rangle$ are mutually orthogonal for $m \neq n$ and that they are (over-) complete for fixed $m=n$ in the quantum phase space of variables $\left(\alpha, \alpha^{*}\right)$ such as the coherent states $|\alpha\rangle$ which are their special case $m=n=0$ with the well-known completeness (over-)relation

$$
\frac{1}{\pi} \int \frac{\mathrm{i}}{2} \mathrm{~d} \alpha \wedge \mathrm{d} \alpha^{*}|\alpha\rangle\langle\alpha|=I \text {. }
$$

Relation (3.22) is a consequence of the more general relation for arbitrary operators $A$ (remind that $\langle A\rangle$ denotes the trace of $A$ )

$$
\frac{1}{\pi} \int \frac{\mathrm{i}}{2} \mathrm{~d} \alpha \wedge \mathrm{d} \alpha^{*} D\left(\alpha, \alpha^{*}\right) A\left(D\left(\alpha, \alpha^{*}\right)\right)^{\dagger}=\langle A\rangle I
$$

We do not derive it here (see, e.g., [9] (chap. I: A coherent state primer) and [10]). In the special cases $A=|n\rangle\langle m| ;(m, n=0,1,2, \cdots)$ follows from (3.25)

$$
\frac{1}{\pi} \int \frac{\mathrm{i}}{2} \mathrm{~d} \alpha \wedge \mathrm{d} \alpha^{*} D\left(\alpha, \alpha^{*}\right)|n\rangle\langle m|\left(D\left(\alpha, \alpha^{*}\right)\right)^{\dagger}=\langle m \mid n\rangle I=\delta_{m, n} I
$$

In (3.19) we defined by $D\left(\alpha, \alpha^{*}\right)|n\rangle \equiv|n, \alpha\rangle$ as the displaced number states. For $n=0, m \neq 0$ relation (25) expresses the overcompleteness of the coherent states $|0, \alpha\rangle \equiv|\alpha\rangle$.

The displaced parity operators possess the trace equal to $\frac{1}{2}$ and are (over-) 
complete in the quantum phase space in the sense described by the resolution of the identity operator $I$

$$
\left\langle\Pi\left(\alpha, \alpha^{*}\right)\right\rangle=\langle\Pi\rangle=\frac{1}{2}, \quad \frac{2}{\pi} \int \frac{\mathrm{i}}{2} \mathrm{~d} \alpha \wedge \mathrm{d} \alpha^{*} \Pi\left(\alpha, \alpha^{*}\right)=I
$$

and they are mutually orthogonal expressed by

$$
\left\langle\Pi\left(\alpha, \alpha^{*}\right) \Pi\left(\beta, \beta^{*}\right)\right\rangle=\frac{\pi}{4} \delta\left(\alpha-\beta, \alpha^{*}-\beta^{*}\right)
$$

Therefore, operators A can be expanded in integrals over phase-space functions as given in (3.2) and the function $A\left(\alpha, \alpha^{*}\right)$ in dependence on $A$ is then determined by the operator $A$ and vice versa.

\section{The Wigner Quasiprobability and Reconstruction of Density Operators}

The Wigner quasiprobability $W\left(\alpha, \alpha^{*}\right)$ in the complex variables $\left(\alpha, \alpha^{*}\right)$ can be defined in (not full) analogy to (3.7) by (remind, $\langle\cdots\rangle$ means trace of content)

$$
\begin{aligned}
W\left(\alpha, \alpha^{*}\right) & =\left\langle\varrho \exp \left(-a \frac{\partial}{\partial \alpha}-a^{\dagger} \frac{\partial}{\partial \alpha^{*}}\right)\right\rangle \delta\left(\alpha, \alpha^{*}\right) \\
& =\frac{2}{\pi}\left\langle\varrho \Pi\left(\alpha, \alpha^{*}\right)\right\rangle, \quad \int \frac{\mathrm{i}}{2} \mathrm{~d} \alpha \wedge \mathrm{d} \alpha^{*} W\left(\alpha, \alpha^{*}\right)=1
\end{aligned}
$$

The reconstruction of the density operator $\varrho$ from it is then determined by

$$
\begin{aligned}
\varrho & =2 \int \frac{\mathrm{i}}{2} \mathrm{~d} \alpha \wedge \mathrm{d} \alpha^{*} W\left(\alpha, \alpha^{*}\right) \Pi\left(\alpha, \alpha^{*}\right) \\
& =\pi \int \frac{\mathrm{i}}{2} \mathrm{~d} \alpha \wedge \mathrm{d} \alpha^{*} W\left(\alpha, \alpha^{*}\right) \exp \left(-a \frac{\partial}{\partial \alpha}-a^{\dagger} \frac{\partial}{\partial \alpha^{*}}\right) \delta\left(\alpha, \alpha^{*}\right)
\end{aligned}
$$

that after partial integration leads to

$$
\begin{aligned}
\varrho & =\pi \int \frac{\mathrm{i}}{2} \mathrm{~d} \alpha \wedge \mathrm{d} \alpha^{*} \delta\left(\alpha, \alpha^{*}\right) \exp \left(a \frac{\partial}{\partial \alpha}+a^{\dagger} \frac{\partial}{\partial \alpha^{*}}\right) W\left(\alpha, \alpha^{*}\right) \\
& =\pi \sum_{k=0}^{\infty} \sum_{l=0}^{\infty} \frac{\mathcal{S}\left\{a^{k} a^{\dagger l}\right\}}{k ! l !}\left\{\frac{\partial^{k+l}}{\partial \alpha^{k} \partial \alpha^{* l}} W\left(\alpha, \alpha^{*}\right)\right\}_{\alpha=\alpha^{*}=0},\langle\varrho\rangle=1
\end{aligned}
$$

The calculation of expectation values $\bar{A} \equiv\langle\varrho A\rangle$ of operators $A$ for density operators $\varrho$ using the Wigner quasiprobability $W\left(\alpha, \alpha^{*}\right)$ has to be made by the formula

$$
\bar{A} \equiv\langle\varrho A\rangle=\int \frac{\mathrm{i}}{2} \mathrm{~d} \alpha \wedge \mathrm{d} \alpha^{*} W\left(\alpha, \alpha^{*}\right) A\left(\alpha, \alpha^{*}\right)=\int \mathrm{d} q \wedge \mathrm{d} p W(q, p) A(q, p)
$$

in analogy to the classical probability theory.

If one compares the relations between classical phase-space functions $A(q, p)$ with the quantum-mechanical equivalent operators $A$ in the WeylWigner formalism in (3.2) and (3.7) with that of the Wigner quasiprobability $W\left(\alpha, \alpha^{*}\right)$ and the reconstruction of the density operator $\varrho$ from $W\left(\alpha, \alpha^{*}\right)$ in (4.1) and in (4.2) then we find a difference in the factors in front. One may be 
astonished about this but it is not very principal and finds a simple historical explanation ${ }^{4}$.

For the transition to the representation by real canonical variable $(q, p)$ and operators $(Q, P)$ one has to use, in particular, the relation

$$
\exp \left(-a \frac{\partial}{\partial \alpha}-a^{\dagger} \frac{\partial}{\partial \alpha^{*}}\right)=\exp \left(-Q \frac{\partial}{\partial q}-P \frac{\partial}{\partial p}\right)
$$

and, furthermore, the relation

$$
\delta\left(\alpha, \alpha^{*}\right)=\delta\left(\frac{q+\mathrm{i} p}{\sqrt{2 \hbar}}, \frac{q-\mathrm{i} p}{\sqrt{2 \hbar}}\right)=2 \hbar \delta(q+\mathrm{i} p, q-\mathrm{i} p)=2 \hbar \delta(q) \delta(p)
$$

Then from (4.1) for the Wigner quasiprobability $W(q, p)$ follows (remind that $\langle\ldots\rangle$ means trace)

$$
W(q, p)=\left\langle\varrho \exp \left(-Q \frac{\partial}{\partial q}-P \frac{\partial}{\partial p}\right)\right\rangle \delta(q) \delta(p), \quad \int \mathrm{d} q \wedge \mathrm{d} p W(q, p)=1
$$

and the reconstruction of the density operator $\varrho$ from the Wigner quasiprobability is possible by

$$
\varrho=2 \pi \hbar \int \mathrm{d} q \wedge \mathrm{d} p W(q, p) \exp \left(-Q \frac{\partial}{\partial q}-P \frac{\partial}{\partial p}\right) \delta(q) \delta(p), \quad\langle\varrho\rangle=1
$$

One may prove then after some calculation that the definition of $W(q, p)$ in (4.7) is fully equivalent to the definition by Wigner [3] (see also [4]) (Wigner denotes it $P(x ; p)$ and generalizes it for several variables to $\left.P\left(x_{1}, \cdots, x_{n} ; p_{1}, \cdots, p_{n}\right)\right)$. In the following, however, we will stay at the representation by complex variables $\left(\alpha, \alpha^{*}\right)$ and will now discuss representations by number and by displaced number states.

\section{Number-State Representations of Displaced Number States Using Laguerre 2D Polynomials}

In the following we derive number-state representations of the relations of the Weyl-Wigner formalism and as a preparation for our next aim we derive the number-state representation of displaced number states. It is advantageous to use for this purpose the Laguerre $2 \mathrm{D}$ polynomials $\mathrm{L}_{m, n}\left(z, z^{*}\right)$ defined as follows (see [17] and citations therein)

$$
\begin{aligned}
\mathrm{L}_{m, n}\left(z, z^{*}\right) & =\exp \left(-\frac{\partial^{2}}{\partial z \partial z^{*}}\right) z^{m} z^{*_{n}} \\
& =(-1)^{m+n} \exp \left(z z^{*}\right) \frac{\partial^{m+n}}{\partial z^{* m} \partial z^{n}} \exp \left(-z z^{*}\right)
\end{aligned}
$$

\footnotetext{
${ }^{4}$ As a quasi probability one is not obliged to accept the normalizations

$\int \frac{i}{2} \mathrm{~d} \alpha \wedge \mathrm{d} \alpha^{*} W\left(\alpha, \alpha^{*}\right)=\int \mathrm{d} q \wedge \mathrm{d} p W(q, p)=1$ such as for genuine probability densities since

$W\left(\alpha, \alpha^{*}\right)$ involves non-orthogonal states of the variables $\left(\alpha, \alpha^{*}\right)$ in its definition but one has in this case also to change the calculation of expectation values in corresponding way. For the transition from the Wigner quasiprobability to a classical distribution function by the limiting procedure $\hbar \rightarrow 0$ it is even favorable to use this normalization but one has before this to make the transition to real canonical variables $(q, p)$ and thus to $W(q, p)$. Therefore, it seems to be unreasonable and not useful to change the established normalization.
} 
and explicitly

$$
\mathrm{L}_{m, n}\left(z, z^{*}\right)=\sum_{j=0}^{\{m, n\}} \frac{(-1)^{j} m ! n !}{j !(m-j) !(n-j) !} z^{m-j} z^{* n-j}
$$

with the following relation to generalized Laguerre (or Laguerre-Sonin) polynomials $\mathrm{L}_{n}^{v}(u)$

$$
\mathrm{L}_{m, n}\left(z, z^{*}\right)=(-1)^{n} n ! z^{m-n} \mathrm{~L}_{n}^{m-n}\left(z z^{*}\right)=(-1)^{m} m ! z^{* n-m} \mathrm{~L}_{m}^{n-m}\left(z z^{*}\right)
$$

The definition of $\mathrm{L}_{m, n}\left(z, z^{*}\right)$ may be generalized to polynomials $\mathrm{L}_{m, n}\left(U ; z, z^{*}\right)$ where $U$ is a two-dimensional unimodular matrix which makes a linear transformation of the two variables [18] that, however, we do not need here.

First, we calculate the expansion of the displaced number states $|\alpha, n\rangle \equiv D\left(\alpha, \alpha^{*}\right)|n\rangle$ defined in (3.19) in number states $|m\rangle$ using the completeness of the number states $\sum_{m=0}^{\infty}|m\rangle\langle m|=I$

$$
|\alpha, n\rangle \equiv \sum_{m=0}^{\infty}|m\rangle\left\langle m\left|D\left(\alpha, \alpha^{*}\right)\right| n\right\rangle
$$

Using the normally ordered representation of the displacement operator in (2.9) we find

$$
\begin{aligned}
& \left\langle m\left|D\left(\alpha, \alpha^{*}\right)\right| n\right\rangle=\exp \left(-\frac{\alpha \alpha^{*}}{2}\right)\left\langle m\left|\exp \left(\alpha a^{\dagger}\right) \exp \left(-\alpha^{*} a\right)\right| n\right\rangle \\
& =\exp \left(-\frac{\alpha \alpha^{*}}{2}\right) \sum_{k=0}^{\infty} \sum_{l=0}^{\infty} \frac{\alpha^{k}\left(-\alpha^{*}\right)^{l}}{k ! l !}\left\langle m\left|a^{\dagger k} a^{l}\right| n\right\rangle \\
& =\exp \left(-\frac{\alpha \alpha^{*}}{2}\right) \sum_{k=0}^{\infty} \sum_{l=0}^{\infty} \frac{(-1)^{l} \sqrt{m ! n !}}{\sqrt{(m-k) !(n-l) ! k ! l !}} \alpha^{k} \alpha^{* l} \underbrace{\langle m-k \mid n-l\rangle}_{=\delta_{m-k, n-l}}
\end{aligned}
$$

and by substitution of the summation indices $(k, l)$ according to $m-k=n-l \equiv j$

$$
\left\langle m\left|D\left(\alpha, \alpha^{*}\right)\right| n\right\rangle=\exp \left(-\frac{\alpha \alpha^{*}}{2}\right) \frac{(-1)^{n}}{\sqrt{m ! n !}} \sum_{j=0}^{\{m, n\}} \frac{(-1)^{j} m ! n !}{j !(m-j) !(n-j) !} \alpha^{m-j} \alpha^{* n-j}
$$

By definition of the Laguerre 2D polynomials in (5.1) this can be written

$$
\begin{aligned}
\left\langle m\left|D\left(\alpha, \alpha^{*}\right)\right| n\right\rangle & =\exp \left(-\frac{\alpha \alpha^{*}}{2}\right) \frac{(-1)^{n}}{\sqrt{m ! n !}} \mathrm{L}_{m, n}\left(\alpha, \alpha^{*}\right) \\
& =\exp \left(-\frac{\alpha \alpha^{*}}{2}\right) \frac{(-1)^{n}}{\sqrt{m ! n !}} \exp \left(-\frac{\partial^{2}}{\partial \alpha \partial \alpha^{*}}\right) \alpha^{m} \alpha^{*_{n}}
\end{aligned}
$$

The expansion (5.4) of displaced number states becomes

$$
|\alpha, n\rangle=\exp \left(-\frac{\alpha \alpha^{*}}{2}\right) \frac{(-1)^{n}}{\sqrt{n !}} \sum_{m=0}^{\infty} \frac{1}{\sqrt{m !}} \mathrm{L}_{m, n}\left(\alpha, \alpha^{*}\right)|m\rangle
$$

For $n=0$ using $\mathrm{L}_{m, 0}\left(\alpha, \alpha^{*}\right)=\alpha^{m}$ one obtains the corresponding expansion of coherent states $|\alpha, 0\rangle \equiv|\alpha\rangle$.

For the product of two displacement operators $\left(D\left(\beta, \beta^{*}\right)\right)^{\dagger}$ and $D\left(\alpha, \alpha^{*}\right)$ one finds from (2.13) 


$$
\begin{aligned}
\left(D\left(\beta, \beta^{*}\right)\right)^{\dagger} D\left(\alpha, \alpha^{*}\right) & =D\left(-\beta,-\beta^{*}\right) D\left(\alpha, \alpha^{*}\right) \\
& =\exp \left(\frac{\alpha \beta^{*}-\alpha^{*} \beta}{2}\right) D\left(\alpha-\beta, \alpha^{*}-\beta^{*}\right)
\end{aligned}
$$

Applying this one obtains for the general scalar product of displaced number states

$$
\begin{aligned}
\langle\beta, m \mid \alpha, n\rangle & =\left\langle m\left|\left(D\left(\beta, \beta^{*}\right)\right)^{\dagger} D\left(\alpha, \alpha^{*}\right)\right| n\right\rangle \\
& =\exp \left(\frac{\alpha \beta^{*}-\alpha^{*} \beta}{2}\right)\left\langle m\left|D\left(\alpha-\beta, \alpha^{*}-\beta^{*}\right)\right| n\right\rangle \\
& =\exp \left(\alpha \beta^{*}-\frac{\alpha \alpha^{*}+\beta \beta^{*}}{2}\right) \frac{(-1)^{n}}{\sqrt{m ! n !}} \mathrm{L}_{m, n}\left(\alpha-\beta, \alpha^{*}-\beta^{*}\right)
\end{aligned}
$$

One sees immediately that for $\beta=\alpha$ using $\mathrm{L}_{m, n}(0,0)=(-1)^{n} n ! \delta_{m, n}$ from this relation follows the orthonormality (3.21) of the displaced number states and for $m=n=0$ using $\mathrm{L}_{0,0}\left(z, z^{*}\right)=1$ the well-known relation for the scalar product of coherent states.

We now derive the representation of the displaced parity operator by number states. From (3.16) and (3.18) follows

$$
\begin{aligned}
\Pi\left(\alpha, \alpha^{*}\right) & =D\left(\alpha, \alpha^{*}\right) \Pi\left(D\left(\alpha, \alpha^{*}\right)\right)^{\dagger}=D\left(2 \alpha, 2 \alpha^{*}\right) \Pi \\
& =\sum_{n=0}^{\infty}(-1)^{n} D\left(2 \alpha, 2 \alpha^{*}\right)|n\rangle\left\langle n\left|=\sum_{n=0}^{\infty}(-1)^{n}\right| 2 \alpha, n\right\rangle\langle n|
\end{aligned}
$$

and using (5.8) with corresponding substitutions

$$
\Pi\left(\alpha, \alpha^{*}\right)=\exp \left(-2 \alpha \alpha^{*}\right) \sum_{m=0}^{\infty} \sum_{n=0}^{\infty} L_{m, n}\left(2 \alpha, 2 \alpha^{*}\right) \frac{|m\rangle\langle n|}{\sqrt{m ! n !}}
$$

Thus we found the following basic number-state representation of the operator $\exp \left(-a \frac{\partial}{\partial \alpha}-a^{\dagger} \frac{\partial}{\partial \alpha^{*}}\right) \delta\left(\alpha, \alpha^{*}\right)$

$$
\exp \left(-a \frac{\partial}{\partial \alpha}-a^{\dagger} \frac{\partial}{\partial \alpha^{*}}\right) \delta\left(\alpha, \alpha^{*}\right)=\frac{2}{\pi} \exp \left(-2 \alpha \alpha^{*}\right) \sum_{m=0}^{\infty} \sum_{n=0}^{\infty} \mathrm{L}_{m, n}\left(2 \alpha, 2 \alpha^{*}\right) \frac{|m\rangle\langle n|}{\sqrt{m ! n !}}(5.1
$$

and the Formulas (3.2) for the transition from a classical phase-space function $A\left(\alpha, \alpha^{*}\right)$ to a quantum-mechanical operator $A=\mathcal{S}\left\{A\left(a, a^{\dagger}\right)\right\}$ in the Weyl-Wigner formalism takes on the form

$$
A=\frac{2}{\pi} \int \frac{\mathrm{i}}{2} \mathrm{~d} \alpha \wedge \mathrm{d} \alpha^{*} A\left(\alpha, \alpha^{*}\right) \exp \left(-2 \alpha \alpha^{*}\right) \sum_{m=0}^{\infty} \sum_{n=0}^{\infty} \mathrm{L}_{m, n}\left(2 \alpha, 2 \alpha^{*}\right) \frac{|m\rangle\langle n|}{\sqrt{m ! n !}} .
$$

The representation of the displaced parity operator and its consequence (5.13) leads also to a convenient representation of the Wigner quasiprobability in the number-state representation. We express the general density operator $\varrho$ by its matrix elements $\langle n|\varrho| m\rangle$ as follows

$$
\varrho=\sum_{m=0}^{\infty} \sum_{n=0}^{\infty}|n\rangle\langle n|\varrho| m\rangle\langle m|
$$

Then the Wigner quasiprobability (4.1) using (5.13) can be represented by the 
following expansion

$$
W\left(\alpha, \alpha^{*}\right)=\frac{2}{\pi} \exp \left(-2 \alpha \alpha^{*}\right) \sum_{m=0}^{\infty} \sum_{n=0}^{\infty} \mathrm{L}_{m, n}\left(2 \alpha, 2 \alpha^{*}\right) \frac{\langle n|\varrho| m\rangle}{\sqrt{m ! n !}}
$$

For its importance we will translate this here also into the representation by canonical variables $(q, p)$ (see (2.1)) with the result

$$
W(q, p)=\frac{1}{\pi \hbar} \exp \left(-\frac{q^{2}+p^{2}}{\hbar}\right) \sum_{m=0}^{\infty} \sum_{n=0}^{\infty} \mathrm{L}_{m, n}\left(\sqrt{\frac{2}{\hbar}}(q+\mathrm{i} p), \sqrt{\frac{2}{\hbar}}(q-\mathrm{i} p)\right) \frac{\langle n|\varrho| m\rangle}{\sqrt{m ! n !}}
$$

in the given normalization $\mathrm{d} q \wedge \mathrm{d} p W(q, p)=1$.

For a displaced number state $|\beta, n\rangle \equiv D\left(\beta, \beta^{*}\right)|n\rangle$ the Wigner quasiprobability $W\left(\alpha, \alpha^{*}\right)$ follows immediately from (5.16) by argument transformation

$$
\begin{aligned}
\left(\alpha, \alpha^{*}\right) \rightarrow(\alpha & \left.-\beta, \alpha^{*}-\beta^{*}\right) \\
W\left(\alpha, \alpha^{*}\right) & =\frac{2}{\pi} \exp \left(-2(\alpha-\beta)\left(\alpha^{*}-\beta^{*}\right)\right) \frac{1}{n !} \mathrm{L}_{n, n}\left(2(\alpha-\beta), 2\left(\alpha^{*}-\beta^{*}\right)\right) \\
& =\frac{2}{\pi} \exp \left(-2(\alpha-\beta)\left(\alpha^{*}-\beta^{*}\right)\right)(-1)^{n} \mathrm{~L}_{n}\left(4(\alpha-\beta)\left(\alpha^{*}-\beta^{*}\right)\right)
\end{aligned}
$$

as the displaced Wigner quasiprobability for a number state $|n\rangle$. Therefore, more generally, if $\varrho$ is the density operator of a displaced state to density operator $\varrho_{0}$ according to

$$
\varrho=D\left(\beta, \beta^{*}\right) \varrho_{0}\left(D\left(\beta, \beta^{*}\right)\right)^{\dagger}
$$

then the corresponding Wigner quasiprobabilities $W\left(\alpha, \alpha^{*}\right)$ and $W_{0}\left(\alpha, \alpha^{*}\right)$ are related by

$$
W\left(\alpha, \alpha^{*}\right)=W_{0}\left(\alpha-\beta, \alpha^{*}-\beta^{*}\right)
$$

that means by a displacement of the arguments.

\section{Quantum-Mechanical Operators Corresponding to Classical Monomial Phase-Space Functions}

We calculate and discuss now the operators $A^{(k, l)}$ which correspond in the Weyl formalism to the basic "classical" phase-space functions $A^{(k, l)}\left(\alpha, \alpha^{*}\right)$ according to

$$
\begin{gathered}
A^{(k, l)}\left(\alpha, \alpha^{*}\right)=\left(\sqrt{\alpha \alpha^{*}}\right)^{k}\left(\sqrt{\frac{\alpha}{\alpha^{*}}}\right)^{l}=\alpha^{\frac{k+l}{2}} \alpha^{* \frac{k-l}{2}}=|\alpha|^{k} \mathrm{e}^{\mathrm{i} l \varphi} \\
A^{(k,-l)}\left(\alpha, \alpha^{*}\right)=\left(A^{(k, l)}\left(\alpha, \alpha^{*}\right)\right)^{*}, \quad(k=0,1,2, \cdots ; l=0, \pm 1, \pm 2, \cdots)
\end{gathered}
$$

in the number representation and express the result by means of the Jacobi polynomials. The functions (6.1) are chosen to include besides the amplitude functions $|\alpha|^{k}$ the basic periodic functions $\mathrm{e}^{\mathrm{i} / \varphi}$ of the phase $\varphi$ of a harmonic oscillator. Expressed by the real canonical variables $(q, p)$ according to $(2.1)$ this corresponds to the complex functions

$$
A^{(k, l)}(q, p) \equiv A^{(k, l)}\left(\frac{q+\mathrm{i} p}{\sqrt{2 \hbar}}, \frac{q-\mathrm{i} p}{\sqrt{2 \hbar}}\right)=\frac{1}{(\sqrt{2 \hbar})^{k}}(q+\mathrm{i} p)^{\frac{k+l}{2}}(q-\mathrm{i} p)^{\frac{k-l}{2}}
$$


Due to factors $(\sqrt{2 \hbar})^{k}$ in the denominator the functions $A^{\prime(k, l)}(q, p)$ are strictly speaking for $k \neq 0$ not genuine classical functions without multiplication by these factors. For positive integer $m=\frac{k+l}{2}$ and $n=\frac{k-l}{2}$ one has the identity

$$
\begin{aligned}
& A^{\prime(m+n, m-n)}(q, p)=\frac{1}{(\sqrt{2 \hbar})^{m+n}}(q+\mathrm{i} p)^{m}(q-\mathrm{i} p)^{n} \\
& =\frac{1}{(\sqrt{2 \hbar})^{m+n}} \sum_{j=0}^{m+n} 2^{j} \mathrm{P}_{j}^{(m-j, n-j)}(0) q^{m+n-j}(\mathrm{i} p)^{j}, \quad(m, n=0,1,2, \cdots)
\end{aligned}
$$

With $\mathrm{P}_{n}^{(\alpha, \beta)}(u)$ we denote the Jacobi polynomials in the now generally accepted definition by Szegö [19] (chap IV) in which they are also programmed in Wolfram's "Mathematica". An older definition with direct reference to Jacobi can be found in [20]. Formulas (6.3) suggests that working with the canonical variables $(q, p)$ one may choose the functions $q^{i} p^{j},(i, j=0,1,2, \cdots)$ as a basis of a space of functions which one may translate into quantum-mechanical symmetrically ordered operators $\mathcal{S}\left\{Q^{i} P^{j}\right\}$ in the sense of Weyl plus distributive law for arbitrary functions of this space. This space of functions, however, is narrower than the space of functions built with the basis functions (6.1). We come back to this at the end of this Section.

We now calculate the quantum-mechanical Weyl equivalents to the basic functions (6.1). Some formal part of these calculations we delegate to Appendix A where we also give the most necessary formulae for the Jacobi polynomials by means of which we represent the results. The calculation of the double integral in (5.14) in Appendix A leads to the following number-state representation of the operator $A^{(k, l)}$ (see (A.2))

$$
\begin{aligned}
A^{(k, l)} & =2^{-\frac{k-l}{2}} \sum_{m=0}^{\infty} \sqrt{m !(m+l) !} \sum_{j=0}^{m} \frac{(-1)^{j}\left(m+\frac{k+l}{2}-j\right) !}{j !(m-j) !(m+l-j) !} 2^{m-j}|m\rangle\langle m+l| \\
& \equiv \mathcal{S}\left\{\left(\sqrt{a a^{\dagger}}\right)^{k}\left(\sqrt{\frac{a}{a^{\dagger}}}\right)^{l}\right\}
\end{aligned}
$$

This may be represented using the Jacobi polynomials $\mathrm{P}_{n}^{(\alpha, \beta)}(u)$ in two alternative forms as follows (already communicated without detailed derivation in [13])

$$
\begin{aligned}
A^{(k, l)} & =2^{-\frac{k-l}{2}}\left(\frac{k+l}{2}\right) ! \sum_{m=0}^{\infty} \sqrt{\frac{m !}{(m+l) !}} \mathrm{P}_{m}^{\left(-m+\frac{k-l}{2}, l\right)}(3)|m\rangle\langle m+l| \\
& =2^{-\frac{k-l}{2}}\left(\frac{k+l}{2}\right) ! \sum_{m=0}^{\infty} \sqrt{\frac{m !}{(m+l) !}} 2^{m} \mathrm{P}_{m}^{\left(-m+\frac{k-l}{2},-m-\frac{k+l}{2}-1\right)}(0)|m\rangle\langle m+l|
\end{aligned}
$$

Explicit representations of the Jacobi polynomials in two different forms are given in (A.3) of Appendix A. The general transformation relation of the Jacobi polynomials specialized from a transformation relation of the Hypergeometric function which lead in our case from argument $u=3$ to argument $u=0$ are 
written down in (A.4). A more detailed treatment of Jacobi polynomials we find besides cited [19], e.g., also in vol. 2 of the monographs of Bateman and Erdélyi [21], in the article of Koornwinder et al. [22] in the NIST Handbook [23] and in our article [24].

There is another transformation relation of the Jacobi polynomials $\mathrm{P}_{n}^{(\alpha, \beta)}(u)$ with integer upper index $\alpha$ or $\beta$ specialized from a corresponding transformation relation of the Hypergeometric function and given in (A.8). It leads from (6.5) to the following essentially different representations

$$
\begin{aligned}
A^{(k, l)} & =2^{-\frac{k+l}{2}}\left(\frac{k-l}{2}\right) ! \sum_{m=0}^{\infty} \sqrt{\frac{(m+l) !}{m !}} \mathrm{P}_{m+l}^{\left(-m+\frac{k-l}{2},-l\right)}(3)|m\rangle\langle m+l| \\
& =2^{-\frac{k+l}{2}}\left(\frac{k-l}{2}\right) ! \sum_{m=0}^{\infty} \sqrt{\frac{(m+l) !}{m !}} 2^{m+l} \mathrm{P}_{m+l}^{\left(-m+\frac{k-l}{2},-m-\frac{k+l}{2}-1\right)}(0)|m\rangle\langle m+l|
\end{aligned}
$$

In comparison to (6.5) it establishes some symmetry by transformations $l \leftrightarrow-l$ and changing then the summation index $m \leftrightarrow n=m+l$ between functions which are involved in these relations as coefficients of $|m\rangle\langle m+l|$ and if one makes the Hermitean conjugation of these relations. All 4 forms (6.5) and (6.6) for $A^{(k, l)}$ are useful since in special cases parts in these formulae become singular and using then the other representations one may avoid limiting considerations. Furthermore, by transformation of $l \rightarrow-l$ the forms (6.5) are transformed into the forms (6.6) and vice versa and one easily proves the conjugation relation

$$
A^{(k,-l)}=\left(A^{(k, l)}\right)^{\dagger}, \quad(l=0, \pm 1, \pm 2, \cdots)
$$

With the Formulas (6.5) and (6.6) we gave four essentially different numberstate representations of the quantum-mechanical (Weyl) equivalents to the classical functions (6.1) by means of the Jacobi polynomials. In some cases one or two of these formulae are not equally appropriate for the calculation of these equivalents because they do not provide the results directly without limit considerations.

We may consider the operators $A^{(k, l)},(k=0,1, \cdots ; l=0, \pm 1, \pm 2, \cdots)$ as basis of a linear space of symmetrically ordered operators with the possibility to add such operators and to multiply them by numbers under validity of the distributive law. Before we discuss special cases of the relations (6.5) and (6.6) we make in generalization of them in next Section a smoothing of the classical functions $\left(\sqrt{\alpha \alpha^{*}}\right)^{k}\left(\sqrt{\frac{\alpha}{\alpha^{*}}}\right)^{l}$ by a normalized Gaussian function and calculate their quantum-mechanical equivalents.

Before implementing the announced programme we establish now the connection between symmetrically ordered powers of operators $\left(a, a^{\dagger}\right)$ and of operators $(Q, P)$. According to (6.3) we have

$$
\mathcal{S}\left\{a^{m} a^{\dagger n}\right\}=\frac{1}{(\sqrt{2 \hbar})^{m+n}} \sum_{j=0}^{m+n}(\mathrm{i} 2)^{j} \mathrm{P}_{j}^{(m-j, n-j)}(0) \mathcal{S}\left\{Q^{m+n-j} P^{j}\right\}
$$


with the inversion

$$
\mathcal{S}\left\{Q^{m} P^{n}\right\}=(\sqrt{2 \hbar})^{m+n}(-\mathrm{i})^{n} \sum_{j=0}^{m+n} \frac{1}{2^{m+n-j}} \mathrm{P}_{j}^{(m-j, n-j)}(0) \mathcal{S}\left\{a^{m+n-j} a^{\dagger j}\right\}
$$

The symmetrically ordered operators $\mathcal{S}\left\{Q^{m} P^{n}\right\}$ can be represented in standard and anti-standard ordering according to

$$
\begin{aligned}
\mathcal{S}\left\{Q^{m} P^{n}\right\} & =\mathcal{S}\left\{P^{n} Q^{m}\right\}=\mathcal{S}\left\{\mathcal{P}\left(Q^{m} P^{n}\right)\right\} \\
& =\sum_{j=0}^{\{m, n\}} \frac{m ! n !}{j !(m-j) !(n-j) !}\left(-\frac{\mathrm{i} \hbar}{2}\right)^{j} Q^{m-j} P^{n-j} \\
& =\sum_{j=0}^{\{m, n\}} \frac{m ! n !}{j !(m-j) !(n-j) !}\left(+\frac{\mathrm{i} \hbar}{2}\right)^{j} P^{n-j} Q^{m-j}
\end{aligned}
$$

where $\mathcal{P}\left(Q^{m} P^{n}\right)$ means an arbitrary permutation of the operators $Q^{m} P^{n}$ in analogy to (2.7).

In the special case $m=n$ using the following special values of the Jacobi polynomials $\mathrm{P}_{j}^{(n-j, n-j)}(u)$ of argument $u=0$

$$
2^{2 k} \mathrm{P}_{2 k}^{(n-2 k, n-2 k)}(0)=\frac{(-1)^{k} n !}{(n-k) ! k !}, \quad 2^{2 k+1} \mathrm{P}_{2 k+1}^{(n-2 k-1, n-2 k-1)}(0)=0
$$

we obtain from $(6.8)$

$$
\mathcal{S}\left\{a^{n} a^{\dagger n}\right\}=\frac{1}{(2 \hbar)^{n}} \sum_{k=0}^{n} \frac{n !}{(n-k) ! k !} \mathcal{S}\left\{Q^{2(n-k)} P^{2 k}\right\}
$$

and from (6.9)

$$
\mathcal{S}\left\{Q^{n} P^{n}\right\}=\left(-\mathrm{i} \frac{\hbar}{2}\right)^{n} \sum_{k=0}^{n} \frac{(-1)^{k} n !}{(n-k) ! k !} \mathcal{S}\left\{a^{2(n-k)} a^{\dagger 2 k}\right\}
$$

with only powers of the squared operators $\left(Q^{2}, P^{2}\right)$ or $\left(a^{2}, a^{\dagger 2}\right)$, respectively, within the ordering symbol $\mathcal{S}\{\ldots\}$ on the right-hand sides. In particular, for $n=1$ we find

$$
\mathcal{S}\left\{a a^{\dagger}\right\}=\frac{1}{2}\left(a a^{\dagger}+a^{\dagger} a\right)=\frac{1}{2 \hbar}\left(Q^{2}+P^{2}\right)=\frac{1}{2 \hbar}\left(\mathcal{S}\left\{Q^{2}\right\}+\mathcal{S}\left\{P^{2}\right\}\right)
$$

and

$$
\mathcal{S}\{Q P\}=\frac{1}{2}(Q P+P Q)=-\mathrm{i} \frac{\hbar}{2}\left(a^{2}-a^{\dagger 2}\right)=-\mathrm{i} \frac{\hbar}{2}\left(\mathcal{S}\left\{a^{2}\right\}-\mathcal{S}\left\{a^{\dagger 2}\right\}\right)
$$

The special values (6.11) for the Jacobi polynomials follow easily from the general expansion (6.3) in case of $m=n$ using the binomial formula.

\section{Quantum-Mechanical Equivalents of Smoothed Classical Functions}

We calculate in this Section the transition from a smoothed classical function of the canonical variables in representation by the complex variables $\left(\alpha, \alpha^{*}\right)$ to its equivalent quantum-mechanical operator in the sense of Weyl. The smoothing of the classical function $A\left(\alpha, \alpha^{*}\right)$ is made by convolution with a normalized 
Gaussian function as follows

$$
A_{r}\left(\alpha, \alpha^{*}\right)=\exp \left(\frac{r}{2} \frac{\partial^{2}}{\partial \alpha \partial \alpha^{*}}\right) A\left(\alpha, \alpha^{*}\right)=\frac{2}{\pi r} \exp \left(-\frac{2 \alpha \alpha^{*}}{r}\right) * A\left(\alpha, \alpha^{*}\right)
$$

where "*" denotes the convolution. It is a smoothing of the function $A\left(\alpha, \alpha^{*}\right)$ for $r>0$. For $r<0$ it is the opposite of smoothing for which we do not find an appropriate word. The equivalence of the right-hand sides in (7.1) is related to the equivalence (3.10) by representing $A\left(\alpha, \alpha^{*}\right)$ as convolution $A\left(\alpha, \alpha^{*}\right)=\delta\left(\alpha, \alpha^{*}\right) * A\left(\alpha, \alpha^{*}\right)$ with a two-dimensional delta function.

According to (5.14) one has now to calculate the equivalent operator $A_{r}$ according to

$$
\begin{aligned}
A_{r}= & \frac{2}{\pi} \int \frac{\mathrm{i}}{2} \mathrm{~d} \alpha \wedge \mathrm{d} \alpha^{*}\left\{\exp \left(\frac{r}{2} \frac{\partial^{2}}{\partial \alpha \partial \alpha^{*}}\right) A\left(\alpha, \alpha^{*}\right)\right\} \\
& \times \exp \left(-2 \alpha \alpha^{*}\right) \sum_{m=0}^{\infty} \sum_{n=0}^{\infty} \mathrm{L}_{m, n}\left(2 \alpha, 2 \alpha^{*}\right) \frac{|m\rangle\langle n|}{\sqrt{m ! n !}} \\
= & \frac{2}{\pi} \int \frac{\mathrm{i}}{2} \mathrm{~d} \alpha \wedge \mathrm{d} \alpha^{*} A\left(\alpha, \alpha^{*}\right)\left\{\exp \left(\frac{r}{2} \frac{\partial^{2}}{\partial \alpha \partial \alpha^{*}}\right)\right. \\
& \left.\times \exp \left(-2 \alpha \alpha^{*}\right) \sum_{m=0}^{\infty} \sum_{n=0}^{\infty} \mathrm{L}_{m, n}\left(2 \alpha, 2 \alpha^{*}\right)\right\} \frac{|m\rangle\langle n|}{\sqrt{m ! n !}}
\end{aligned}
$$

where we applied partial integration.

Let us first make a remark. The smoothing with an operator $\exp \left(\frac{r}{2} \frac{\partial^{2}}{\partial \alpha \partial \alpha^{*}}\right)$ does not lead in all cases of $r \neq 0$ to a new function $A_{r}\left(\alpha, \alpha^{*}\right) \neq A\left(\alpha, \alpha^{*}\right)$. Due to $\frac{\partial^{2}}{\partial \alpha \partial \alpha^{*}} \alpha^{k}=0$ and $\frac{\partial^{2}}{\partial \alpha \partial \alpha^{*}} \alpha^{* k}=0$ we have

$$
\exp \left(\frac{r}{2} \frac{\partial^{2}}{\partial \alpha \partial \alpha^{*}}\right) \alpha^{k}=\alpha^{k}, \quad \exp \left(\frac{r}{2} \frac{\partial^{2}}{\partial \alpha \partial \alpha^{*}}\right) \alpha^{* l}=\alpha^{* l}, \quad(k, l=0,1,2, \cdots)
$$

Therefore, the smoothed quantum-mechanical equivalents $\left(a^{k}\right)_{r}$ and $\left(a^{\dagger k}\right)_{r}$ for $r \neq 0$ are not different from $a^{k}$ and $a^{\dagger k}$, respectively. One may look to this also in the following way. The operators $\exp \left(\frac{r}{2} \frac{\partial^{2}}{\partial \alpha \partial \alpha^{*}}\right),(-\infty<r<+\infty)$ form an Abelian (i.e., commutative) one-parameter Lie group and as basis for (in general, reducible) representations may serve functions $A\left(\alpha, \alpha^{*}\right)$ of (very general) function spaces. The functions $\alpha^{k}$ and $\alpha^{* k}$ in (7.3) form a basis of the function space for the identical representation of the mentioned group. Products of genuine powers of functions $\alpha$ and $\alpha^{*}$ do not belong to this last function space since, for example

$$
\frac{\partial^{2}}{\partial \alpha \partial \alpha^{*}} \alpha \alpha^{*}=1 \Rightarrow \exp \left(\frac{r}{2} \frac{\partial^{2}}{\partial \alpha \partial \alpha^{*}}\right) \alpha \alpha^{*}=\alpha \alpha^{*}+\frac{r}{2}
$$

More generally, we find for the smoothed functions $\left(\alpha^{k} \alpha^{* l}\right)_{r}$ to the 
functions $\alpha^{k} \alpha^{* l},(k, l=0,1,2, \cdots)$ by Taylor-series expansion of the operator

$$
\begin{aligned}
\exp \left(\frac{r}{2} \frac{\partial^{2}}{\partial \alpha \partial \alpha^{*}}\right) & \\
\left(\alpha^{k} \alpha^{* l}\right)_{r} & \equiv \exp \left(\frac{r}{2} \frac{\partial^{2}}{\partial \alpha \partial \alpha^{*}}\right) \alpha^{k} \alpha^{* l}=\sum_{j=0}^{\{k, l\}} \frac{k ! l !}{j !(k-j) !(l-j) !}\left(\frac{r}{2}\right)^{j} \alpha^{k-j} \alpha^{* l-j}
\end{aligned}
$$

Without more detailed discussion (see, e.g., [15]) we mention that for $r=1$ we get antinormally ordered operators $\mathcal{A}\left\{a^{k} a^{\dagger l}\right\}$ if the corresponding operators $A$ are the symmetrically ordered operators $\mathcal{S}\left\{a^{k} a^{\dagger l}\right\}$ and similarly the symmetrically ordered operators $\mathcal{S}\left\{a^{k} a^{\dagger l}\right\}$ if the corresponding operators $A$ are the normally ordered operators $\mathcal{N}\left\{a^{k} a^{\dagger l}\right\}=a^{\dagger l} a^{k}$ according to

$$
\begin{aligned}
\mathcal{A}\left\{a^{k} a^{\dagger l}\right\} & =\sum_{j=0}^{\{k, l\}} \frac{k ! l !}{j !(k-j) !(l-j) !} \underbrace{\mathcal{N}\left\{a^{k-j} a^{\dagger l-j}\right\}}_{=a^{\dagger l-j} a^{k-j}} \\
& =\sum_{j=0}^{\{k, l\}} \frac{k ! l !}{j !(k-j) !(l-j) !}\left(\frac{1}{2}\right)^{j} \mathcal{S}\left\{a^{k-j} a^{\dagger l-j}\right\} \equiv a^{k} a^{\dagger l} \\
\mathcal{S}\left\{a^{k} a^{\dagger l}\right\} & =\sum_{j=0}^{\{k, l\}} \frac{k ! l !}{j !(k-j) !(l-j) !}\left(\frac{1}{2}\right)^{j} \underbrace{\mathcal{N}\left\{a^{k-j} a^{\dagger l-j}\right\}}_{=a^{\dagger l-j} a^{k-j}} \equiv \mathcal{S}\left\{a^{\dagger l} a^{k}\right\}
\end{aligned}
$$

These formulae can be represented by the Laguerre $2 \mathrm{D}$ polynomials (2) but with the imaginary unit " $\mathrm{i}$ " in their arguments.

In quantum optics there is often used a class of smoothed $(r>0)$ quasiprobabilities $F_{r}\left(\alpha, \alpha^{*}\right)$ ([7] ( $r=-s$ there), and, e.g., [10]) according to ( $r$ mostly restricted to $-1 \leq r \leq+1$ )

$$
\begin{aligned}
& F_{r}\left(\alpha, \alpha^{*}\right) \equiv \exp \left(\frac{r}{2} \frac{\partial^{2}}{\partial \alpha \partial \alpha^{*}}\right) W\left(\alpha, \alpha^{*}\right), \\
& F_{+1}\left(\alpha, \alpha^{*}\right) \equiv Q\left(\alpha, \alpha^{*}\right), \quad F_{-1}\left(\alpha, \alpha^{*}\right) \equiv P\left(\alpha, \alpha^{*}\right)
\end{aligned}
$$

The class of quasiprobabilities $F_{r}\left(\alpha, \alpha^{*}\right)$ does not belong to function classes which for different $r$ may take on the same functions since $\alpha^{k}$ and $\alpha^{* k}$ cannot be quasiprobabilities to density operators $\varrho$ with trace equal to 1 (the traces of the operators $a^{k}$ and $a^{\dagger k}$ are 0). Expectation values of smoothed operators $A_{r}$ can be calculated with the "smoothed" quasiprobability $F_{-r}\left(\alpha, \alpha^{*}\right)$ according to (remind that $\langle\ldots\rangle$ means the trace)

$$
\begin{aligned}
\langle\varrho A\rangle & =\int \frac{\mathrm{i}}{2} \mathrm{~d} \alpha \wedge \mathrm{d} \alpha^{*} W\left(\alpha, \alpha^{*}\right) A\left(\alpha, \alpha^{*}\right) \\
& =\int \frac{\mathrm{i}}{2} \mathrm{~d} \alpha \wedge \mathrm{d} \alpha^{*} W\left(\alpha, \alpha^{*}\right)\left\{\exp \left(-\frac{r}{2} \frac{\partial^{2}}{\partial \alpha \partial \alpha^{*}}\right) A_{r}\left(\alpha, \alpha^{*}\right)\right\} \\
& =\int \frac{\mathrm{i}}{2} \mathrm{~d} \alpha \wedge \mathrm{d} \alpha^{*}\left\{\exp \left(-\frac{r}{2} \frac{\partial^{2}}{\partial \alpha \partial \alpha^{*}}\right) W\left(\alpha, \alpha^{*}\right)\right\} A_{r}\left(\alpha, \alpha^{*}\right)
\end{aligned}
$$

where we used partial integration. This provides with definition (7.7)

$$
\langle\varrho A\rangle=\int \frac{\mathrm{i}}{2} \mathrm{~d} \alpha \wedge \mathrm{d} \alpha^{*} F_{-r}\left(\alpha, \alpha^{*}\right) A_{r}\left(\alpha, \alpha^{*}\right)
$$


On the other side the expectation value of the smoothed operator $A_{r}$ can be calculated using the function $A_{r}\left(\alpha, \alpha^{*}\right)$ together with the Wigner quasiprobability $W\left(\alpha, \alpha^{*}\right)$ according to

$$
\begin{aligned}
\left\langle\varrho A_{r}\right\rangle & =\int \frac{\mathrm{i}}{2} d \alpha \wedge d \alpha^{*} W\left(\alpha, \alpha^{*}\right) A_{r}\left(\alpha, \alpha^{*}\right) \\
& =\int \frac{\mathrm{i}}{2} d \alpha \wedge d \alpha^{*} F_{r}\left(\alpha, \alpha^{*}\right) A\left(\alpha, \alpha^{*}\right)
\end{aligned}
$$

The second form is obtained from the first form by partial integration. Formulas (7.9) and (7.10) equip us with different possibilities to calculate the expectation values $\bar{A} \equiv\langle\varrho A\rangle$ and $\overline{A_{r}} \equiv\left\langle\varrho A_{r}\right\rangle$.

\section{Explicit Expressions for the Quantum-Mechanical Weyl Equivalents to Smoothed Classical Monomial Phase-Space Functions}

We now calculate the equivalent quantum-mechanical operators $A_{r}^{(k, l)}$ to the smoothed classical functions $A_{r}^{(k, l)}\left(\alpha, \alpha^{*}\right)$ according to (7.1) by means of the Formulas (7.2). The detailed calculations are represented in Appendix B. The smoothed functions $A_{r}^{(k, l)}\left(\alpha, \alpha^{*}\right)$ possess the explicit form as a series expansions

$$
A_{r}^{(k, l)}\left(\alpha, \alpha^{*}\right)=\sum_{j=0}^{\infty} \frac{\left(\frac{k+l}{2}\right) !\left(\frac{k-l}{2}\right) !}{j !\left(\frac{k+l}{2}-j\right) !\left(\frac{k-l}{2}-j\right) !}\left(\frac{r}{2}\right)^{j} \alpha^{\frac{k+l}{2}-j} \alpha^{* \frac{k-l}{2}-j}
$$

For both integer $\frac{k+l}{2}$ and integer $\frac{k-l}{2}$ the right-hand side can be represented by the Laguerre $2 \mathrm{D}$ polynomials (5.2). It is easy to check that for $r=0$ one obtains the function $A^{(k, l)}\left(\alpha, \alpha^{*}\right)=\alpha^{\frac{k+l}{2}} \alpha^{* \frac{k-l}{2}}$ and for $l=k$ and $l=-k$ the functions $A_{r}^{(k, k)}\left(\alpha, \alpha^{*}\right)=\alpha^{k}$ and $A_{r}^{(k,-k)}\left(\alpha, \alpha^{*}\right)=\alpha^{* k}$, respectively, which are independent of the parameter $r$ as discussed in the previous Section. However, Formulas (8.1) is problematic for cases when $\frac{k+l}{2}$ or $\frac{k-l}{2}$ is not a non-negative integer which restricts the sum over $j$ to a finite sum since in the other cases one has to investigate the character of the convergence of the infinite sum over $j$ which in the neighborhood of $\alpha=\alpha^{*}=0$ and for $\alpha=\alpha^{*} \rightarrow \infty$ is not guaranteed.

As the first step we obtained in Appendix B as generalization of (6.4)

$$
\begin{aligned}
A_{r}^{(k, l)}= & \left(\frac{2}{1+r}\right)^{-\frac{k-l}{2}} \sum_{m=0}^{\infty} \sqrt{m !(m+l) !}\left(\frac{1-r}{1+r}\right)^{m} \\
& \times\left(\sum_{j=0}^{m} \frac{(-1)^{j}\left(m+\frac{k+l}{2}-j\right) !}{j !(m-j) !(m+l-j) !}\left(\frac{2}{1-r}\right)^{m-j}\right)|m\rangle\langle m+l|
\end{aligned}
$$


This can be represented using the Jacobi polynomials in the following two equivalent ways

$$
\begin{aligned}
& A_{r}^{(k, l)}=\left(\frac{2}{1+r}\right)^{-\frac{k-l}{2}}\left(\frac{k+l}{2}\right) ! \sum_{m=0}^{\infty} \sqrt{\frac{m !}{(m+l) !}}\left(\frac{1-r}{1+r}\right)^{m} \mathrm{P}_{m}^{\left(-m+\frac{k-l}{2}, l\right)}\left(\frac{3+r}{1-r}\right)|m\rangle\langle m+l| \\
& =\left(\frac{2}{1+r}\right)^{-\frac{k-l}{2}}\left(\frac{k+l}{2}\right) ! \sum_{m=0}^{\infty} \sqrt{\frac{m !}{(m+l) !}}\left(\frac{2}{1+r}\right)^{m} \mathrm{P}_{m}^{\left(-m+\frac{k-l}{2},-m-\frac{k+l}{2}-1\right)}(-r)|m\rangle\langle m+l|
\end{aligned}
$$

in generalization of (6.6). Alternatively, using the relation (A.8) this can be also represented in the form

$$
\begin{aligned}
& A_{r}^{(k, l)}=\left(\frac{2}{1+r}\right)^{-\frac{k+l}{2}}\left(\frac{k-l}{2}\right) ! \sum_{m=0}^{\infty} \sqrt{\frac{(m+l) !}{m !}}\left(\frac{1-r}{1+r}\right)^{m+l} \mathrm{P}_{m+l}^{\left(-m+\frac{k-l}{2},-l\right)}\left(\frac{3+r}{1-r}\right)|m\rangle\langle m+l| \\
& =\left(\frac{2}{1+r}\right)^{-\frac{k+l}{2}}\left(\frac{k-l}{2}\right) ! \sum_{m=0}^{\infty} \sqrt{\frac{(m+l) !}{m !}}\left(\frac{2}{1+r}\right)^{m+l} \mathrm{P}_{m+l}^{\left(-m+\frac{k-l}{2},-m-\frac{k+l}{2}-1\right)}(-r)|m\rangle\langle m+l|
\end{aligned}
$$

showing some symmetry of (8.3) to (8.4) under substitutions $m \rightarrow m^{\prime}=m+l, l \rightarrow l^{\prime}=-l$ and Hermitean adjunction. All alternative forms in (8.3) and (8.3) are useful because some in special cases become undetermined but the others in these cases, as a rule, can be used without limiting procedures.

In special case $r=0$ we get the Formulas (6.5) and (6.6), respectively, setting $A_{r=0}^{(k, l)} \equiv A^{(k, l)}$.

In special case $r=1$ Formulas (8.2) and the first parts in (8.3) and (8.4) become indeterminate and have to be dealt with by the limiting transition $r \rightarrow 1$ but from the second parts we find without limiting procedure

$$
\begin{aligned}
A_{r=1}^{(k, l)} & =\left(\frac{k+l}{2}\right) ! \sum_{m=0}^{\infty} \sqrt{\frac{m !}{(m+l) !}} \mathrm{P}_{m}^{\left(-m+\frac{k-l}{2},-m-\frac{k+l}{2}-1\right)}(-1)|m\rangle\langle m+l| \\
& =\left(\frac{k-l}{2}\right) ! \sum_{m=0}^{\infty} \sqrt{\frac{(m+l) !}{m !}} \mathrm{P}_{m+l}^{\left(-m+\frac{k-l}{2},-m-\frac{k+l}{2}-1\right)}(-1)|m\rangle\langle m+l|
\end{aligned}
$$

Using the Formulas (A.6) for the Jacobi polynomials of argument $u=-1$ observing the decomposition $\left(-m-\frac{k+l}{2}-1\right)=\left(-m-l-\frac{k-l}{2}-1\right)$ and applying the general relation $\frac{(-1)^{k}(-\gamma) !}{(-k-\gamma) !}=\frac{(k+\gamma-1) !}{(\gamma-1) !}$ for integer $k$ we obtain from both relations (8.5)

$$
A_{r=1}^{(k, l)}=\sum_{m=0}^{\infty} \frac{\left(m+\frac{k+l}{2}\right) !}{\sqrt{m !(m+l) !}}|m\rangle\langle m+l| \equiv \mathcal{N}\left\{a^{+\frac{k+l}{2}} a^{\frac{k-l}{2}}\right\}
$$

The same result can be also calculated by applying the Formulas (B.9) with the specialization (6.1) of the functions $A\left(\alpha, \alpha^{*}\right)$ or simpler by limiting transition $r \rightarrow 1$ in (8.2). We mention yet that for $r \rightarrow-1$ the above formulae (8.2), (8.3) and (8.4) possess a singularity and $A_{r \rightarrow-1}^{(k, l)}$ becomes genuinely singular. 
In (7.3) we found that smoothing of the functions $\alpha^{k}$ and $\alpha^{* k}$ with normalized Gaussian functions does not influence these functions. It is interesting to consider this from the point of view of our general formulae (8.3) and (8.4). Therefore, we consider now the special case $l=k$ of the classical function $A^{(k, l)}\left(\alpha, \alpha^{*}\right)$

$$
A_{r}^{(k, k)}\left(\alpha, \alpha^{*}\right)=A^{(k, k)}\left(\alpha, \alpha^{*}\right)=\alpha^{k} \Leftrightarrow A_{r}^{(k, k)}=A^{(k, k)}=a^{k},(k=0,1,2, \cdots)
$$

which shows some interesting aspects. As result for the corresponding operators $A_{r}^{(k, k)}$ which do not depend on the parameter $r$ we find in number representation

$$
A_{r}^{(k, k)}=A^{(k, k)}=\sum_{m=0}^{\infty} \sqrt{\frac{(m+k) !}{m !}}|m\rangle\langle m+k|=a^{k} \equiv \mathcal{N}\left\{a^{k}\right\}
$$

On the other side from (8.3) follows

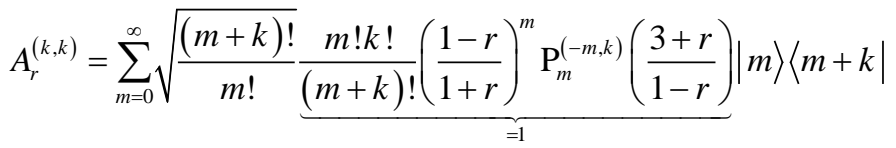

$$
\begin{aligned}
& =\sum_{m=0}^{\infty} \sqrt{\frac{(m+k) !}{m !}} \frac{m ! k !}{(m+k) !}\left(\frac{2}{1+r}\right)_{=1}^{m} \mathrm{P}_{m}^{(-m,-m-k-1)}(-r)|m\rangle\langle m+k|
\end{aligned}
$$

and, in analogous way, from (8.4)

$$
\begin{aligned}
A_{r}^{(k, k)} & =\sum_{m=0}^{\infty} \sqrt{\frac{(m+k) !}{m !}} \underbrace{\left.\frac{1-r}{1+r}\right)^{m+k}\left(\frac{1+r}{2}\right)^{k} \mathrm{P}_{m+k}^{(-m,-k)}\left(\frac{3+r}{1-r}\right)}_{=1}|m\rangle\langle m+k| \\
& =\sum_{m=0}^{\infty} \sqrt{\frac{(m+k) !}{m !}} \underbrace{\left(\frac{2}{1+r}\right)^{m} \mathrm{P}_{m+k}^{(-m,-m-k-1)}(-r)}_{=1}|m\rangle\langle m+k|
\end{aligned}
$$

We see here that from the knowledge of the result (8.8) we find identities for special classes of Jacobi polynomials which are representable in these cases by simple expressions and, clearly, can be derived also in pure mathematical way from explicit representations of the Jacobi polynomials by finite sums (e.g., second line in (A.3) or third line in (A.3) together with the symmetry (A.5)).

In a widely analogous way we may consider the special case $l=-k$ of the classical functions

$A_{r}^{(k,-k)}\left(\alpha, \alpha^{*}\right)=A^{(k,-k)}\left(\alpha, \alpha^{*}\right)=\alpha^{* k} \Leftrightarrow A_{r}^{(k,-k)}=A^{(k,-k)}=a^{\dagger k},(k=0,1,2, \cdots)$

which leads to

$$
A_{r}^{(k,-k)}=A^{(k,-k)}=\sum_{m=0}^{\infty} \sqrt{\frac{(m+k) !}{m !}}|m+k\rangle\langle m|=a^{\dagger k} \equiv \mathcal{N}\left\{a^{\dagger k}\right\}
$$

which is independent on the smoothing parameter $r$ and provides us evaluations for special cases of the Jacobi polynomials if we do not know them already from direct considerations. 


\section{Classical Amplitude Functions and Their Equivalent Quantum-Mechanical Weyl Operators}

We now investigate the equivalent quantum-mechanical operators to amplitude functions $A_{r}^{(k, 0)}\left(\alpha, \alpha^{*}\right)$ according to

$$
A_{r}^{(k, 0)}\left(\alpha, \alpha^{*}\right)=|\alpha|^{k}=\left(\alpha \alpha^{*}\right)^{\frac{k}{2}}, \quad A^{\prime(k, 0)}(q, p)=\left(\sqrt{\frac{q^{2}+p^{2}}{2 \hbar}}\right)^{k}
$$

Due to $(\sqrt{\hbar})^{k}$ in the denominator of $A^{\prime(k, 0)}(q, p)$ the transition $\hbar \rightarrow 0$ of Planck's constant $\hbar \equiv \frac{h}{2 \pi}$ is not possible and the quantum-mechanical operators $A_{r}^{(k, 0)}$ do not possess a direct correspondence to classical functions of the canonical variables without multiplication by $(\sqrt{\hbar})^{k}$. From (8.2) follows for $l=0$ and arbitrary smoothing parameter $r$

$$
A_{r}^{(k, 0)}=\left(\frac{2}{1+r}\right)^{-\frac{k}{2}} \sum_{m=0}^{\infty} m !\left(\frac{1-r}{1+r}\right)^{m}\left(\sum_{j=0}^{m} \frac{(-1)^{j}\left(m+\frac{k}{2}-j\right) !}{j !(m-j) !^{2}}\left(\frac{2}{1-r}\right)^{m-j}\right)|m\rangle\langle m|
$$

with the special case of the identity operator $I$ for $k=0$

$$
A_{r}^{(0,0)}=\sum_{m=0}^{\infty}|m\rangle\langle m|=I
$$

for arbitrary smoothing parameters $r$. The operators (9.2) are diagonal in the number states and are therefore functions of the number operator $N$ alone. Expressed by the Jacobi polynomials both Formulas (8.3) and (8.4) provide in this case the same representations as follows

$$
\begin{aligned}
A_{r}^{(k, 0)} & =\left(\frac{2}{1+r}\right)^{-\frac{k}{2}}\left(\frac{k}{2}\right) ! \sum_{m=0}^{\infty}\left(\frac{1-r}{1+r}\right)^{m} \mathrm{P}_{m}^{\left(-m+\frac{k}{2}, 0\right)}\left(\frac{3+r}{1-r}\right)|m\rangle\langle m| \\
& =\left(\frac{2}{1+r}\right)^{-\frac{k}{2}}\left(\frac{k}{2}\right) ! \sum_{m=0}^{\infty}\left(\frac{2}{1+r}\right)^{m} \mathrm{P}_{m}^{\left(-m+\frac{k}{2},-m-\frac{k}{2}-1\right)}(-r)|m\rangle\langle m|
\end{aligned}
$$

For smoothing of $A^{(k, 0)}$ with parameter $r=1$ follows (see also (8.6))

$$
A_{r=1}^{(k, 0)}=\sum_{m=0}^{\infty} \frac{\left(m+\frac{k}{2}\right) !}{m !}|m\rangle\langle m|=\frac{\left(N+\frac{k}{2} I\right) !}{N !}
$$

The two cases $k=1$ and $k=2$ are illustrated in Figure 1 .

For even $k=2 l$ we have the following decomposition of $A_{r=1}^{(2 l, 0)}$ in powers of the operator $N$

$$
A_{r=1}^{(2 l, 0)}=\frac{(N+I I) !}{N !}=\sum_{j=0}^{l}(-1)^{j} \mathrm{~s}(l+1, l+1-j) N^{l-j}
$$

where $s(k, l)$ are the Stirling numbers of first kind (e.g, [25] [26], from decomposition of $\frac{x !}{(x-k) !}$ in powers of $\left.x\right)$. For odd numbers $k=2 l+1$ it is 

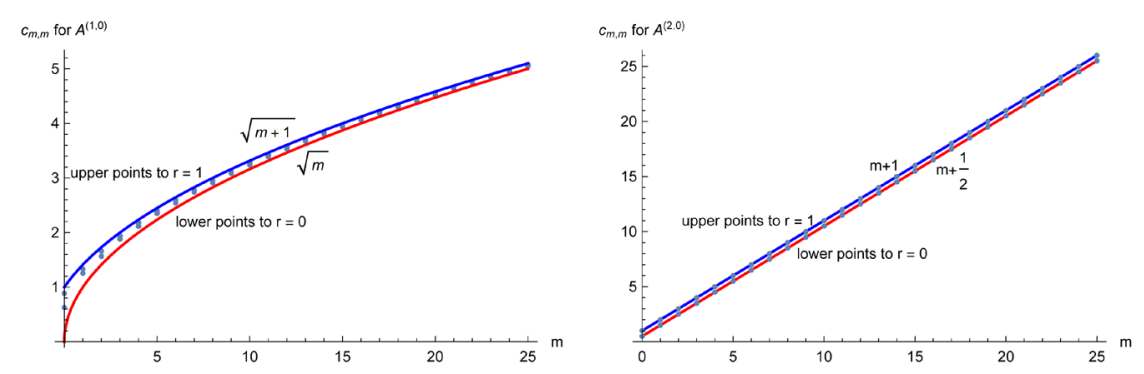

Figure 1. C oefficients $c_{m, m}$ in $A_{r}^{(1,0)}=\sum_{m=0}^{\infty} c_{m, m}|m\rangle\langle m|$ and of $A_{r}^{(2,0)}=\sum_{m=0}^{\infty} c_{m, m}|m\rangle\langle m|$ from (9.4). The upper points are for the smoothed operators $A_{r=1}^{(1,0)}$ but apart from the first they are difficult to distinguish for $A_{r=1}^{(1,0)}$ from the points for $A^{(1,0)}$ in the chosen scale. It is easy to generate these figures from the formulae in a larger scale.

not possible to find a finite decomposition only in powers of $N$.

The case $r=0$ (that means without smoothing) of the Weyl correspondence follows from (9.2) $\left(A^{(k, 0)} \equiv A_{r=0}^{(k, 0)}\right)$

$$
A^{(k, 0)}=2^{-\frac{k}{2}} \sum_{m=0}^{\infty} m !\left(\sum_{j=0}^{m} \frac{(-1)^{j}\left(m+\frac{k}{2}-j\right) !}{j !(m-j) !^{2}} 2^{m-j}\right)|m\rangle\langle m|
$$

and expressed by the Jacobi polynomials, alternatively

$$
\begin{aligned}
A^{(k, 0)} & =2^{-\frac{k}{2}}\left(\frac{k}{2}\right) ! \sum_{m=0}^{\infty} \mathrm{P}_{m}^{\left(-m+\frac{k}{2}, 0\right)}(3)|m\rangle\langle m| \\
& =2^{-\frac{k}{2}}\left(\frac{k}{2}\right) ! \sum_{m=0}^{\infty} 2^{m} \mathrm{P}_{m}^{\left(-m+\frac{k}{2},-m-\frac{k}{2}-1\right)}(0)|m\rangle\langle m|
\end{aligned}
$$

The Formulas (9.7) and (9.8) can be extended from integer $k$ to arbitrary real $k=\kappa$ since the upper indices $(\alpha, \beta)$ of the general Jacobi polynomials $\mathrm{P}_{n}^{(\alpha, \beta)}(z)$ can be arbitrary real (or even complex) numbers [21].

For even $k=2 k^{\prime}$ one obtains from (9.7) and (9.8)

$$
A^{\left(2 k^{\prime}, 0\right)}=\frac{1}{2^{l}} \sum_{m=0}^{\infty} m !\left(\sum_{j=0}^{m} \frac{(-1)^{j}\left(m+k^{\prime}-j\right) !}{j !(m-j) !^{2}}\right)|m\rangle\langle m|
$$

and, in particular, for $k^{\prime}=1$

$$
A^{(2,0)}=\sum_{m=0}^{\infty}\left(m+\frac{1}{2}\right)|m\rangle\langle m|=N+\frac{1}{2} I \equiv \mathcal{S}\left\{a^{\dagger} a\right\}
$$

and for $k^{\prime}=2$

$$
A^{(4,0)}=\sum_{m=0}^{\infty}\left\{\left(m+\frac{1}{2}\right)^{2}+\frac{1}{4}\right\}|m\rangle\langle m|=\left(N+\frac{1}{2}\right)^{2}+\frac{1}{4} I \equiv \mathcal{S}\left\{a^{\dagger 2} a^{2}\right\}
$$

More general relations for $\mathcal{S}\left\{a^{\dagger k} a^{k}\right\}$ are given in Section 12 and in Sequence 1 and Sequence 2 of Appendix C.

In the special case $k=1$ we find for the Jacobi polynomials $\mathrm{P}_{m}^{\left(-m+\frac{1}{2}, 0\right)}(3)$ 
where we have to distinguish the even case $m=2 n$ and the odd case $m=2 n+1$

$$
\begin{gathered}
\mathrm{P}_{2 n}^{\left(-2 n+\frac{1}{2}, 0\right)}(3)=2^{2 n} \mathrm{P}_{2 n}^{\left(-2 n+\frac{1}{2},-2 n-\frac{3}{2}\right)}(0)=(4 n+1) \frac{(2 n) !}{2^{2 n} n !^{2}} \\
\mathrm{P}_{2 n+1}^{\left(-2 n-\frac{1}{2}, 0\right)}(3)=2^{2 n+1} \mathrm{P}_{2 n+1}^{\left(-2 n-\frac{1}{2},-2 n-\frac{5}{2}\right)}(0)=2(2 n+1) \frac{(2 n) !}{2^{2 n} n !^{2}}
\end{gathered}
$$

and from (9.8) follows

$$
\begin{aligned}
A^{(1,0)} & =\sqrt{\frac{\pi}{2}} \sum_{n=0}^{\infty} \frac{(2 n) !}{2^{2 n} n !^{2}}\left\{\left(2 n+\frac{1}{2}\right)|2 n\rangle\langle 2 n|+(2 n+1)| 2 n+1\rangle\langle 2 n+1|\right\} \\
& \equiv \mathcal{S}\left\{\sqrt{a^{\dagger} a}\right\}
\end{aligned}
$$

In the special case $k=3$ we find for the Jacobi polynomials $\mathrm{P}_{m}^{\left(-m+\frac{3}{2}, 0\right)}(3)$ where again we have to distinguish the even case $m=2 n$ and the odd case $m=2 n+1$

$$
\begin{gathered}
\mathrm{P}_{2 n}^{\left(-2 n+\frac{3}{2}, 0\right)}(3)=2^{2 n} \mathrm{P}_{2 n}^{\left(-2 n+\frac{3}{2},-2 n-\frac{5}{2}\right)}(0)=\frac{32 n^{2}+16 n+3}{3} \frac{(2 n) !}{2^{2 n} n !^{2}} \\
\mathrm{P}_{2 n+1}^{\left(-2 n+\frac{1}{2}, 0\right)}(3)=2^{2 n+1} \mathrm{P}_{2 n+1}^{\left(-2 n+\frac{1}{2},-2 n-\frac{7}{2}\right)}(0)=\frac{4(4 n+3)(2 n+1)}{3} \frac{(2 n) !}{2^{2 n} n !^{2}}
\end{gathered}
$$

and from (9.8) follows

$$
\begin{aligned}
& A^{(3,0)} \\
& =\sqrt{\frac{\pi}{2}} \sum_{n=0}^{\infty} \frac{(2 n) !}{2^{2 n} n !^{2}}\left\{\frac{32 n^{2}+16 n+3}{8}|2 n\rangle\left\langle 2 n\left|+\frac{(4 n+3)(2 n+1)}{2}\right| 2 n+1\right\rangle\langle 2 n+1|\right\} \\
& \equiv \mathcal{S}\left\{\sqrt{a^{\dagger 3} a^{3}}\right\}
\end{aligned}
$$

It is difficult to find such explicit forms for higher odd $k$.

One may even calculate the case $k=-1$ according to the Formulas (9.8). For the Jacobi polynomials $\mathrm{P}_{m}^{\left(-m-\frac{1}{2}, 0\right)}(3)$ we find

$$
\begin{gathered}
P_{2 n}^{\left(-2 n-\frac{1}{2}, 0\right)}(3)=2^{2 n} P_{2 n}^{\left(-2 n-\frac{1}{2},-2 n-\frac{1}{2}\right)}(0)=\frac{(2 n) !}{2^{2 n} n !^{2}} \\
P_{2 n+1}^{\left(-2 n-\frac{3}{2}, 0\right)}(3)=2^{2 n+1} P_{2 n+1}^{\left(-2 n-\frac{3}{2},-2 n-\frac{3}{2}\right)}(0)=0
\end{gathered}
$$

and we obtain

$$
A^{(-1,0)}=\sqrt{2 \pi} \sum_{n=0}^{\infty} \frac{(2 n) !}{2^{2 n} n !^{2}}|2 n\rangle\left\langle 2 n\left|=\sqrt{2} \sum_{n=0}^{\infty} \frac{\left(n-\frac{1}{2}\right) !}{n !}\right| 2 n\right\rangle\langle 2 n| \equiv \mathcal{S}\left\{\frac{1}{\sqrt{a^{\dagger} a}}\right\}
$$

We have here only the even number states involved.

\section{Classical Periodic Phase Functions and Their Equivalent Quantum-Mechanical Weyl Operators}

After the amplitude functions we now investigate the equivalent quantummechanical operators to periodic phase functions $A_{r}^{(0, l)}\left(\alpha, \alpha^{*}\right)$ according to 


$$
\begin{gathered}
A^{(0, l)}\left(\alpha, \alpha^{*}\right)=\mathrm{e}^{\mathrm{i} l \varphi}=\left(\frac{\alpha}{\alpha^{*}}\right)^{\frac{l}{2}}, \quad A^{\prime(0, l)}(q, p)=\left(\sqrt{\frac{q+\mathrm{i} p}{q-\mathrm{i} p}}\right)^{l} \\
A^{(0,-l)}\left(\alpha, \alpha^{*}\right)=\mathrm{e}^{-\mathrm{i} l \varphi}=\left(\mathrm{e}^{\mathrm{i} l \varphi}\right)^{*}
\end{gathered}
$$

The expression $A^{\prime(0, l)}(q, p)$ does not contain Planck's constant $\hbar$ and the limiting transition setting $\hbar=0$ is possible but number states are nonclassical. From (8.2) follows for $k=0$ and arbitrary $r$

$$
\begin{aligned}
& A_{r}^{(0, l)}=\left(\frac{2}{1+r}\right)^{\frac{l}{2}} \sum_{m=0}^{\infty} \sqrt{m !(m+l) !}\left(\frac{1-r}{1+r}\right)^{m} \\
& \times\left(\sum_{j=0}^{m} \frac{(-1)^{j}\left(m+\frac{l}{2}-j\right) !}{j !(m-j) !(m+l-j) !}\left(\frac{2}{1-r}\right)^{m-j}\right)|m\rangle\langle m+l| \\
& A_{r}^{(0,-l)}=\left(A_{r}^{(0, l)}\right)^{\dagger}
\end{aligned}
$$

For $l \neq 0$ it is "non-diagonal" in the number-state representation.

If we express (10.2) by the Jacobi polynomials we find from (8.3) the representations

$$
\begin{aligned}
A_{r}^{(0, l)} & =\left(\frac{2}{1+r}\right)^{\frac{l}{2}}\left(\frac{l}{2}\right) ! \sum_{m=0}^{\infty} \sqrt{\frac{m !}{(m+l) !}}\left(\frac{1-r}{1+r}\right)^{m} \mathrm{P}_{m}^{\left(-m-\frac{l}{2}, l\right)}\left(\frac{3+r}{1-r}\right)|m\rangle\langle m+l| \\
& =\left(\frac{2}{1+r}\right)^{\frac{l}{2}}\left(\frac{l}{2}\right) ! \sum_{m=0}^{\infty} \sqrt{\frac{m !}{(m+l) !}}\left(\frac{2}{1+r}\right)^{m} \mathrm{P}_{m}^{\left(-m-\frac{l}{2},-m-\frac{l}{2}-1\right)}(-r)|m\rangle\langle m+l|
\end{aligned}
$$

or alternatively from (8.4)

$$
\begin{aligned}
A_{r}^{(0, l)} & =\left(\frac{2}{1+r}\right)^{-\frac{l}{2}}\left(-\frac{l}{2}\right) ! \sum_{m=0}^{\infty} \sqrt{\frac{(m+l) !}{m !}}\left(\frac{1-r}{1+r}\right)^{m+l} \mathrm{P}_{m+l}^{\left(-m-\frac{l}{2},-l\right)}\left(\frac{3+r}{1-r}\right)|m\rangle\langle m+l| \\
& =\left(\frac{2}{1+r}\right)^{-\frac{l}{2}}\left(-\frac{l}{2}\right) ! \sum_{m=0}^{\infty} \sqrt{\frac{(m+l) !}{m !}}\left(\frac{2}{1+r}\right)^{m+l} \mathrm{P}_{m+l}^{\left(-m-\frac{l}{2},-m-\frac{l}{2}-1\right)}(-r)|m\rangle\langle m+l|
\end{aligned}
$$

For the Weyl correspondence which corresponds to $r=0$ we find from $(10.3)$

$$
\begin{aligned}
A^{(0, l)} & =2^{\frac{1}{2}}\left(\frac{l}{2}\right) ! \sum_{m=0}^{\infty} \sqrt{\frac{m !}{(m+l) !}} \mathrm{P}_{m}^{\left(-m-\frac{l}{2}, l\right)}(3)|m\rangle\langle m+l| \\
& =2^{\frac{1}{2}}\left(\frac{l}{2}\right) ! \sum_{m=0}^{\infty} \sqrt{\frac{m !}{(m+l) !}} 2^{m} \mathrm{P}_{m}^{\left(-m-\frac{l}{2},-m-\frac{l}{2}-1\right)}(0)|m\rangle\langle m+l|
\end{aligned}
$$

or alternatively

$$
\begin{aligned}
A^{(0, l)} & =2^{-\frac{l}{2}}\left(-\frac{l}{2}\right) ! \sum_{m=0}^{\infty} \sqrt{\frac{(m+l) !}{m !}} \mathrm{P}_{m}^{\left(-m--^{2},-l\right)}(3)|m\rangle\langle m+l| \\
& =2^{-\frac{l}{2}}\left(-\frac{l}{2}\right) ! \sum_{m=0}^{\infty} \sqrt{\frac{(m+l) !}{m !}} 2^{m+l} \mathrm{P}_{m+l}^{\left(-m-\frac{l}{2},-m-\frac{l}{2}-1\right)}(0)|m\rangle\langle m+l|
\end{aligned}
$$


The Jacobi polynomials to argument $u=3$ or to $u=0$ with the present upper indices in (10.5) or in (10.6) can be expressed by simple formulae of multiplicative type (see Appendix A, Equation (A.10)) but we have to distinguish the cases of even $m=2 n$ and of odd $m=2 n+1$ of the degree of the polynomials. This leads to the following general formula for arbitrary integer $l$ and $r=0$ (i.e., not smoothed)

$$
\begin{aligned}
A^{(0, l)}= & \sum_{n=0}^{\infty} \sqrt{\frac{\left(n+\frac{l}{2}\right) !}{n !}}\left\{\sqrt{\frac{\left(n-\frac{1}{2}\right) !}{\left(n+\frac{l-1}{2}\right) !}}|2 n\rangle\langle 2 n+l|\right. \\
& +\sqrt{\left.\frac{\left(n+\frac{1}{2}\right) !}{\left(n+\frac{l+1}{2}\right) !}|2 n+1\rangle\langle 2 n+1+l|\right\}}
\end{aligned}
$$

The two cases $l=1$ and $l=2$ are illustrated in Figure 2 .

The Formulas (10.2) for $r=0$ can also be obtained by integrating the Wigner quasiprobability $W\left(\alpha, \alpha^{*}\right)$ over the radius $|\alpha|$ in polar coordinates $(|\alpha|, \varphi)$. One obtains in this way observing the generality of the density operator $\varrho$

$$
\begin{aligned}
W(\varphi) & \equiv \int_{0}^{+\infty} \mathrm{d}|\alpha||\alpha| W\left(|\alpha| \mathrm{e}^{\mathrm{i} \varphi},|\alpha| \mathrm{e}^{-\mathrm{i} \varphi}\right) \\
& =\frac{1}{2 \pi} \sum_{l=-\infty}^{+\infty} \mathrm{e}^{-\mathrm{i} l \varphi}\left\langle\varrho A^{(0, l)}\right\rangle, \quad \int_{-\pi}^{+\pi} \mathrm{d} \varphi W(\varphi)=1
\end{aligned}
$$

where $\langle\ldots\rangle$ denotes the trace of the content in brackets and $A^{(0, l)}$ are the operators explicitly given in equivalent representations in (10.5) and (10.6). The right-hand side of (10.8) possesses the form of the Fourier decomposition of the $2 \pi$-periodic function $W(\varphi)$ with Fourier coefficients $\left\langle\varrho A^{(0, l)}\right\rangle$ determined by

$$
\left\langle\varrho A^{(0, l)}\right\rangle=\int_{0}^{2 \pi} \mathrm{d} \varphi W(\varphi) \mathrm{e}^{\mathrm{i} l \varphi}, \quad(l=0, \pm 1, \pm 2, \cdots)
$$
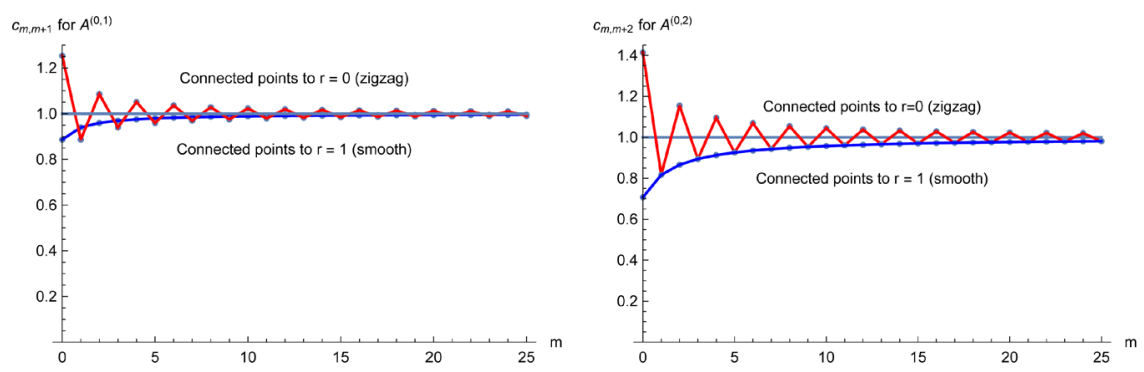

Figure 2. C oefficients $c_{m, m+l}$ in $A^{(0,1)}=\sum_{m=0}^{\infty} c_{m, m+1}|m\rangle\langle m+1|$ and in $A^{(0,2)}=\sum_{m=0}^{\infty} c_{m, m+2}|m\rangle\langle m+2|$. This corresponds to classical phase functions $\mathrm{e}^{\mathrm{i} \varphi}$ and $\mathrm{e}^{\mathrm{i} 2 \varphi}$, correspondingly. The formulae for the quantum-mechanical equivalents are given in (10.7) (for $r=0$ ) and in (10.10) (for smoothed case $r=1$ ). In the SusskindGlogower formalism (see (11.9)) this corresponds to the operators $E_{-}$and $E_{-}^{2}$. 
This is the way in which it was first derived by Garraway and Knight [14] (see also Peřinová, A. Lukš and J. Peřina [12]). In our derivation the operators $A^{(0, l)}$ are embedded together with the operators $A^{(k, 0)}$ into the representation of a more general class of operators $A^{(k, l)}$ with a more general number-state representation by means of the Jacobi polynomials.

In special case $r=1$ of smoothed functions (10.1) (see also (8.1)) follows from the second of the representations in (10.3) or from (8.6)

$$
A_{r=1}^{(0, l)}=\sum_{m=0}^{\infty} \frac{\left(m+\frac{l}{2}\right) !}{\sqrt{m !(m+l) !}}|m\rangle\langle m+l|
$$

This formula can also be obtained by integration of the Husimi-Kano quasiprobability $Q\left(\alpha, \alpha^{*}\right)=\frac{1}{\pi}\langle\alpha|\varrho| \alpha\rangle$ over the radius $|\alpha|$ in polar coordinates coordinates $(|\alpha|, \varphi)$ according to

$$
\begin{aligned}
Q(\varphi) & \equiv \int_{0}^{+\infty} \mathrm{d}|\alpha||\alpha| Q\left(|\alpha| \mathrm{e}^{\mathrm{i} \varphi},|\alpha| \mathrm{e}^{-\mathrm{i} \varphi}\right) \\
& =\frac{1}{2 \pi} \sum_{l=-\infty}^{+\infty} \mathrm{e}^{-\mathrm{i} l \varphi}\left\langle\varrho A_{r=1}^{(0, l)}\right\rangle \geq 0, \quad \int_{-\pi}^{+\pi} \mathrm{d} \varphi Q(\varphi)=1
\end{aligned}
$$

in analogy to (10.8) as the Fourier series of the $2 \pi$-periodic function $Q(\varphi)$ with the Fourier coefficients

$$
\left\langle\varrho A_{r=1}^{(0, l)}\right\rangle=\int_{0}^{2 \pi} \mathrm{d} \varphi Q(\varphi) \mathrm{e}^{\mathrm{i} l \varphi}=\sum_{m=0}^{\infty} \frac{\left(m+\frac{l}{2}\right) !}{\sqrt{m !(m+l) !}}\langle m+l|\varrho| m\rangle,(l=0, \pm 1, \pm 2, \cdots)
$$

in analogy to (10.9).

The phase space distributions $W(\varphi)$ and $Q(\varphi)$ are normalized as given in (10.8) and (10.11) but they are quasiprobabilities. The function $W(\varphi)$ can take on negative values depending on the states whereas $Q(\varphi)$ is non-negative but, nevertheless, it is a quasiprobability because it involves the non-orthogonal coherent states for its definition. As an example, we calculate their explicit forms for coherent states $|\beta\rangle$ with the quasiprobabilities

$$
F_{r}\left(\alpha, \alpha^{*}\right)=\frac{2}{(1+r) \pi} \exp \left(-\frac{2(\alpha-\beta)\left(\alpha^{*}-\beta^{*}\right)}{1+r}\right)
$$

with $F_{0}\left(\alpha, \alpha^{*}\right)=W\left(\alpha, \alpha^{*}\right), F_{1}\left(\alpha, \alpha^{*}\right)=Q\left(\alpha, \alpha^{*}\right) \quad$ and $\quad F_{-1}\left(\alpha, \alpha^{*}\right)=P\left(\alpha, \alpha^{*}\right)$. From this one finds by integration over the modulus of $|\alpha|$ for coherent states $|\beta\rangle$ with $\left(\beta, \beta^{*}\right)=\left(|\beta| \mathrm{e}^{\mathrm{i} \chi},|\beta| \mathrm{e}^{-\mathrm{i} \chi}\right)$

$$
\begin{aligned}
F_{r}(\varphi)= & \frac{1}{2 \pi} \exp \left(-\frac{2}{1+r}|\beta|^{2}\right)\left\{1+\sqrt{\frac{2 \pi}{1+r}}|\beta| \cos (\varphi-\chi)\right. \\
& \left.\cdot \exp \left(\frac{2}{1+r}|\beta|^{2} \cos ^{2}(\varphi-\chi)\right) \cdot\left(1+\operatorname{Erf}\left(\sqrt{\frac{2}{1+r}}|\beta| \cos (\varphi-\chi)\right)\right)\right\}
\end{aligned}
$$


with the special cases $F_{0}(\varphi)=W(\varphi), F_{1}(\varphi)=Q(\varphi)$ and $F_{-1}(\varphi)=P(\varphi)$ where the last becomes singular and has to be considered as a generalized function. Since $F_{r}\left(\alpha, \alpha^{*}\right)$ for $1 \geq r>-1$ is positive definite the functions $F_{r}(\varphi)$ are also positive definite for these parameter values.

We mention here that the information contained in $Q(\varphi)$ cannot be directly obtained from the function $W(\varphi)$ and that for this purpose the complete function $W\left(\alpha, \alpha^{*}\right)$ is necessary since a part of this information is already destroyed in $W(\varphi)$. The relation between the Wigner quasiprobability $W\left(\alpha, \alpha^{*}\right)$ and the quasiprobabilities $F_{r}\left(\alpha, \alpha^{*}\right)$ is (see (7.7), "*” means convolution)

$$
\begin{aligned}
F_{r}\left(\alpha, \alpha^{*}\right) & =\exp \left(\frac{r}{2} \frac{\partial^{2}}{\partial \alpha \partial \alpha^{*}}\right) W\left(\alpha, \alpha^{*}\right)=\frac{2}{r \pi} \exp \left(-\frac{2 \alpha \alpha^{*}}{r}\right) * W\left(\alpha, \alpha^{*}\right) \\
& \equiv \frac{2}{r \pi} \int \frac{\mathrm{i}}{2} \mathrm{~d} \beta \wedge \mathrm{d} \beta^{*} \exp \left(-\frac{2(\alpha-\beta)\left(\alpha^{*}-\beta^{*}\right)}{r}\right) W\left(\beta, \beta^{*}\right)
\end{aligned}
$$

One finds $F_{r}(\varphi)$ from this by integration $\int_{0}^{+\infty} \mathrm{d}|\alpha||\alpha| F_{r}\left(|\alpha| \mathrm{e}^{\mathrm{i} \varphi},|\alpha| \mathrm{e}^{-\mathrm{i} \varphi}\right)$ with $\left(\beta, \beta^{*}\right)=\left(|\beta| \mathrm{e}^{\mathrm{i} \chi},|\beta| \mathrm{e}^{-\mathrm{i} \chi}\right)$

$$
\begin{aligned}
F_{r}(\varphi)= & \frac{1}{2 \pi} \int_{-\pi}^{+\pi} \mathrm{d} \chi \int_{0}^{+\infty} \mathrm{d}|\beta||\beta| \exp \left(-\frac{2|\beta|^{2}}{r}\right) \\
& \cdot\left\{1+\sqrt{\frac{2 \pi}{r}}|\beta| \cos (\varphi-\chi) \exp \left(\frac{2|\beta|^{2} \cos ^{2}(\varphi-\chi)}{r}\right)\right. \\
& \left.\cdot\left(1+\operatorname{Erf}\left(\frac{\sqrt{2}|\beta| \cos (\varphi-\chi)}{\sqrt{r}}\right)\right)\right\} W\left(|\beta| \mathrm{e}^{\mathrm{i} \chi},|\beta| \mathrm{e}^{-\mathrm{i} \chi}\right)
\end{aligned}
$$

For $r=1$ one obtains the special case $F_{1}(\varphi)=Q(\varphi)$. Formulas (10.16) shows that it is not possible to get a direct relation between, for example, $Q(\varphi)$ and $W(\varphi)$ without knowing the more general function $W\left(\alpha, \alpha^{*}\right)$. This is different from the functions $Q\left(\alpha, \alpha^{*}\right)$ and $W\left(\alpha, \alpha^{*}\right)$ which both contain the same complete information over the state only coded in different way.

\section{About the Algebra of the Weyl Correspondences to Classical Phase-Space Functions}

As system of basis operators for a quantum-mechanical harmonic oscillator the operators $A^{(k, l)}$ defined in (6.4) are overcomplete since already each set of operators $\mathcal{N}\left\{a^{\dagger k} a^{l}\right\} \equiv a^{\dagger k} a^{l}, \mathcal{S}\left\{a^{\dagger k} a^{l}\right\}, \mathcal{A}\left\{a^{\dagger k} a^{l}\right\} \equiv a^{l} a^{\dagger k}$ with $k, l=0,1,2, \cdots$ is appropriate as basic set for the expansion of arbitrary non-singular operators in connection with the distributive law.

For the products of classical functions (6.1) we have the following relations

$$
A^{(k, l)}\left(\alpha, \alpha^{*}\right) A^{\left(k^{\prime}, l^{\prime}\right)}\left(\alpha, \alpha^{*}\right)=A^{\left(k+k^{\prime}, l+l^{\prime}\right)}\left(\alpha, \alpha^{*}\right)
$$

The quantum-mechanical equivalent operators do not satisfy analogous relations and instead we find from (6.6) 


$$
\begin{aligned}
A^{(k, l)} A^{\left(k^{\prime}, l^{\prime}\right)}= & 2^{-\frac{k+k^{\prime}-l-l^{\prime}}{2}}\left(\frac{k+l}{2}\right) !\left(\frac{k^{\prime}+l^{\prime}}{2}\right) ! \sum_{m=0}^{\infty} \sqrt{\frac{m !}{\left(m+l+l^{\prime}\right) !}} \\
& \cdot \mathrm{P}_{m}^{\left(-m+\frac{k-l}{2}, l\right)}(3) \mathrm{P}_{m+l}^{\left(-m-l+\frac{k^{\prime}-l^{\prime}}{2}, l^{\prime}\right)}(3)|m\rangle\left\langle m+l+l^{\prime}\right| \\
& \neq A^{\left(k^{\prime}, l^{\prime}\right)} A^{(k, l)}
\end{aligned}
$$

that means that the products $A^{(k, l)} A^{\left(k^{\prime}, l^{\prime}\right)}$ are, in general, noncommutative and therefore also in contrast to the classical equality (11.1) that they are not equal, in general, to $A^{\left(k+k^{\prime},+l^{\prime}\right)}$ that means

$$
\begin{aligned}
A^{\left(k+k^{\prime}, l+l^{\prime}\right)=} & 2^{-\frac{k+k^{\prime}-l-l^{\prime}}{2}}\left(\frac{k+k^{\prime}+l+l^{\prime}}{2}\right) ! \sum_{m=0}^{\infty} \sqrt{\frac{m !}{\left(m+l+l^{\prime}\right) !}} \\
& \cdot \mathrm{P}_{m}^{\left(-m+\frac{k+k^{\prime}-l-l^{\prime}}{2}, l+l^{\prime}\right)}(3)|m\rangle\left\langle m+l+l^{\prime}\right| \\
\neq & A^{(k, l)} A^{\left(k^{\prime}, l^{\prime}\right)}
\end{aligned}
$$

plus possible further representations using the alternative representations for $A^{(k, l)}$. However these products are associative according to

$$
\left(A^{(k, l)} A^{\left(k^{\prime}, l^{\prime}\right)}\right) A^{\left(k^{\prime \prime}, l^{\prime \prime}\right)}=A^{(k, l)}\left(A^{\left(k^{\prime}, l^{\prime}\right)} A^{\left(k^{\prime \prime}, l^{\prime}\right)}\right) \equiv A^{(k, l)} A^{\left(k^{\prime}, l^{\prime}\right)} A^{\left(k^{\prime \prime}, l^{\prime \prime}\right)}
$$

This follows from the associativity of the products $|m\rangle\left\langle m+l \mid m^{\prime}\right\rangle\left\langle m^{\prime}+l^{\prime} \mid m^{\prime \prime}\right\rangle\left\langle m^{\prime \prime}+l^{\prime \prime}\right|$ in the arising triple sum over $m, m^{\prime}, m^{\prime \prime}$.

In general, the operators $A^{(k, l)}$ and $A^{\left(k^{\prime}, l^{\prime}\right)}$ do not commute. Apart from the trivial cases $k=k^{\prime}, l=l^{\prime}$ the operators $A^{(k, l)}$ and $A^{\left(k^{\prime}, l^{\prime}\right)}$ commute also for $l=l^{\prime}=0$ according to

$$
\begin{aligned}
A^{(k, 0)} A^{\left(k^{\prime}, 0\right)} & =2^{-\frac{k+k^{\prime}}{2}}\left(\frac{k}{2}\right) !\left(\frac{k^{\prime}}{2}\right) ! \sum_{m=0}^{\infty} \mathrm{P}_{m}^{\left(-m+\frac{k}{2}, 0\right)}(3) \mathrm{P}_{m}^{\left(-m+\frac{k^{\prime}}{2}, 0\right)}(3)|m\rangle\langle m| \\
& =A^{\left(k^{\prime}, 0\right)} A^{(k, 0)} \neq A^{\left(k+k^{\prime}, 0\right)}
\end{aligned}
$$

In these cases the operators $A^{(k, 0)}$ and $A^{\left(k^{\prime}, 0\right)}$ and thus their products $A^{(k, 0)} A^{\left(k^{\prime}, 0\right)}$ are diagonal in the number representation but generally their products $A^{(k, 0)} A^{\left(k^{\prime}, 0\right)}$ are not equal to $A^{\left(k+k^{\prime}, 0\right)}$. According to (8.8) and (8.11) in the special cases $l=k$ and $l=-k$ the operators $A^{(k, l)}$ are $A^{(k, k)}=a^{k}$ and $A^{(k,-k)}=a^{\dagger k}$, respectively. Since smoothing of these operators does not change them we can extend this behavior to arbitrary smoothing parameter $r$ and taking into account (8.9) or (8.10)

$$
\begin{aligned}
A_{r}^{(k, k)} A_{r^{\prime}}^{(l, l)} & =\sum_{m=0}^{\infty} \sum_{n=0}^{\infty} \sqrt{\frac{(m+k) !(n+l) !}{m ! n !}}|m\rangle\langle m+k \mid n\rangle\langle n+l| \\
& =\sum_{m=0}^{\infty} \sqrt{\frac{(m+k+l) !}{m !}}|m\rangle\langle m+k+l|=A_{r^{\prime \prime}}^{(k+l, k+l)}
\end{aligned}
$$

for arbitrary $r, r^{\prime}$ and $r^{\prime \prime}$ and, analogously, taking into account (8.12)

$$
A_{r}^{(k,-k)} A_{r^{\prime}}^{(l,-l)}=A_{r^{\prime \prime}}^{(k+l,-k-l)}
$$

In cases when $A^{(k, l)}$ and $A^{\left(k^{\prime}, l^{\prime}\right)}$ do not commutate one may calculate the commutator from the given relations and may express it by means of the Jacobi polynomials. For example, for the commutator of $A^{(0,1)}$ corresponding to 
classical $\mathrm{e}^{\mathrm{i} \varphi}$ with $A^{(0,-1)}=\left(A^{(0,1)}\right)^{\dagger}$ follows from (10.7)

$$
\left[A^{(0,1)}, A^{(0,-1)}\right]=\sum_{n=0}^{\infty} \frac{\left(n-\frac{1}{2}\right) !}{2 n !}\left\{\frac{\left(n-\frac{1}{2}\right) !}{n !}|2 n\rangle\left\langle 2 n\left|-\frac{\left(n+\frac{1}{2}\right) !}{(n+1) !}\right| 2 n+1\right\rangle\langle 2 n+1|\right\}
$$

In the Susskind-Glogower formalism [12] [27] [28], for comparison, we have for the analogous operators $E_{-}$and $E_{+}=\left(E_{-}\right)^{\dagger}$ (correspondence $A^{(0,1)} \sim E_{-} \sim \mathrm{e}^{\mathrm{i} \varphi}$ and $\left.A^{(0,-1)} \sim E_{+} \sim \mathrm{e}^{-\mathrm{i} \varphi}\right)$

$$
\begin{gathered}
E_{-} \equiv \sum_{m=0}^{\infty}|m\rangle\left\langle m+1\left|, \quad E_{+} \equiv \sum_{m=0}^{\infty}\right| m+1\right\rangle\left\langle m\left|, \quad\left[E_{-}, E_{+}\right]=\right| 0\right\rangle\langle 0| \\
\left(E_{-}\right)^{l}=\sum_{m=0}^{\infty}|m\rangle\left\langle m+l\left|, \quad\left(E_{+}\right)^{l}=\sum_{m=0}^{\infty}\right| m+l\right\rangle\left\langle m\left|, \quad\left[\left(E_{-}\right)^{l},\left(E_{+}\right)^{l}\right]=\sum_{n=0}^{l-1}\right| n\right\rangle\langle n|
\end{gathered}
$$

For explicit calculation this formalism is often simpler than using the formulae in Section 10 resulting from the Weyl correspondence of classical to quantum optics. However, one cannot make in this formalism a distinction between symmetrical Weyl ordering and normal ordering. To find a simple general mathematical relation between these two approaches seems to be difficult. It is also easier to deal with the eigenvalue problems (right-hand eigenstates) for the operators $\left(E_{-}\right)^{l}$ than the corresponding eigenvalue problems for the operators $A_{r}^{(0, l)}$.

\section{Powers of the Classical Intensity and Their Equivalent Quantum-Mechanical Weyl Operators}

The classical intensity is by definition $A A^{*}$ if $A$ is the complex amplitude of a considered process (e.g., harmonic oscillator). We made in (6.1), (6.2) and (9.1) the agreement (not also with some disadvantages) to "normalize" it using the Planck constant $\hbar$ to get in the Weyl correspondence directly the symmetrized product of annihilation and creation operator $\mathcal{S}\left\{a a^{\dagger}\right\}$ connected with the number operator $N \equiv a^{\dagger} a$. For its $k$-th powers we have according to (9.1) the "classical" function in representation by canonical variables $(q, p)$ and in complex variables $\left(\alpha, \alpha^{*}\right)$ (substitute $k^{\prime} \rightarrow k$ in (9.9))

$$
A^{(2 k, 0)}\left(\alpha, \alpha^{*}\right)=|\alpha|^{2 k}=\left(\alpha \alpha^{*}\right)^{k}, \quad A^{\prime(2 k, 0)}(q, p)=\left(\frac{q^{2}+p^{2}}{2 \hbar}\right)^{k}
$$

with the quantum-mechanical equivalent (smoothing parameter is here $r=0$ )

$$
A^{(2 k, 0)}=\mathcal{S}\left\{a^{k} a^{\dagger k}\right\} \equiv \mathcal{S}\left\{a^{\dagger k} a^{k}\right\}
$$

According to the meaning of the symbol for symmetrical ordering $\mathcal{S}\{\ldots\}$ the ordering of the annihilation and creation operators within the braces is arbitrary.

We now derive the relations between symmetrical ordering $\mathcal{S}\{\ldots\}$ and normal ordering $\mathcal{N}\{\ldots\}$ for products of equal numbers of annihilation and 
creation operators that means for combinations where the phase of these operators is fully eliminated. From (7.6) follows for the special case $k=l$

$$
\begin{aligned}
\mathcal{S}\left\{a^{\dagger k} a^{k}\right\} & =\sum_{j=0}^{k} \frac{k !^{2}}{j !(k-j) !^{2}}\left(\frac{1}{2}\right)^{j} a^{\dagger k-j} a^{k-j} \\
& \equiv \sum_{j=0}^{k} \frac{k !^{2}}{j !(k-j) !^{2}}\left(\frac{1}{2}\right)^{j} \mathcal{N}\left\{a^{\dagger k-j} a^{k-j}\right\}
\end{aligned}
$$

The inversion of this relation is

$$
\mathcal{N}\left\{a^{\dagger k} a^{k}\right\} \equiv a^{\dagger k} a^{k}=\sum_{j=0}^{k} \frac{k !^{2}}{j !(k-j) !^{2}}\left(-\frac{1}{2}\right)^{j} \mathcal{S}\left\{a^{\dagger k-j} a^{k-j}\right\}
$$

This can be proved analogously to corresponding more general formulae for $a^{\dagger k} a^{l}$ by inserting one of the Formulas (12.3) and (12.4) into the other one and using after a simple transformation of the arising double sum the binomial formula. In particular, we find from (12.3)

$$
\begin{gathered}
\mathcal{S}\left\{a^{\dagger} a\right\}=a^{\dagger} a+\frac{1}{2} I=N+\frac{1}{2} I \\
\mathcal{S}\left\{a^{\dagger 2} a^{2}\right\}=a^{\dagger 2} a^{2}+2 a^{\dagger} a+\frac{1}{2} I=\left(N+\frac{1}{2} I\right)^{2}+\frac{1}{4} I=\left(\mathcal{S}\left\{a^{\dagger} a\right\}\right)^{2}+\frac{1}{4} I
\end{gathered}
$$

The explicit form for more initial special cases is given in Appendix C. The inversion of (12.5) could be immediately written down from the analogous structure of the relations (12.3) and (12.4) with changing signs. In Appendix C we also derive general representations of the symmetrically ordered operators $\mathcal{S}\left\{a^{\dagger k} a^{k}\right\}$ by powers of the number operator $N$ and by powers of the operator $N+\frac{1}{2} I=\mathcal{S}\left\{a^{\dagger} a\right\}$ and give them explicitly for a few initial cases.

We now derive from (12.5) an inequality for expectation values. For this purpose we use the Cauchy-Bunyakovski-Schwarz inequality for states $|\psi\rangle$ and $|\varphi\rangle$ in Hilbert space or for operators $A$ and $B$ in a Hilbert space of operators in the forms

$$
\begin{aligned}
& \langle\psi \mid \psi\rangle\langle\varphi \mid \varphi\rangle \geq\langle\psi \mid \varphi\rangle\langle\varphi \mid \psi\rangle=\langle\psi \mid \varphi\rangle\langle\psi \mid \varphi\rangle^{*} \\
& \left\langle A^{\dagger} A\right\rangle\left\langle B^{\dagger} B\right\rangle \geq\left\langle A^{\dagger} B\right\rangle\left\langle B^{\dagger} A\right\rangle=\left\langle A^{\dagger} B\right\rangle\left\langle A^{\dagger} B\right\rangle^{*}
\end{aligned}
$$

From the second equation in (12.5) follows (remind that overlining means forming the expectation value and $\langle C\rangle$ forming of the trace of an arbitrary operator $C$ )

$$
\begin{aligned}
\overline{\mathcal{S}\left\{a^{\dagger 2} a^{2}\right\}} & \equiv\left\langle\varrho \mathcal{S}\left\{a^{\dagger 2} a^{2}\right\}\right\rangle \underbrace{\langle\varrho\rangle}_{=1} \\
& =\left\langle\sqrt{\varrho}\left(N+\frac{1}{2} I\right)\left(N+\frac{1}{2} I\right) \sqrt{\varrho}\right\rangle\langle\sqrt{\varrho} \sqrt{\varrho}\rangle+\frac{1}{4}\langle\varrho I\rangle \\
& \geq\left\langle\sqrt{\varrho}\left(N+\frac{1}{2} I\right) \sqrt{\varrho}\right\rangle^{2}+\frac{1}{4}=\overline{\left(N+\frac{1}{2} I\right)}^{2}+\frac{1}{4} \equiv{\overline{\mathcal{S}\left\{a^{\dagger} a\right.}}^{2}+\frac{1}{4}
\end{aligned}
$$


The operator $\sqrt{\varrho}$ is here defined as a positive semi-definite Hermitean operator that is possible since the density operator $\varrho$ itself is also a positive semi-definite Hermitean operator.

From (12.7) we see the inequality

$$
\overline{\mathcal{S}\left\{a^{\dagger 2} a^{2}\right\}}-{\overline{\mathcal{S}}\left\{a^{\dagger} a\right\}}^{2}=\overline{(\Delta N)^{2}}+\frac{1}{4} \geq \frac{1}{4}
$$

with the usual definition of the operator $\Delta N$

$$
\Delta N \equiv N-\bar{N} I \Rightarrow \overline{\Delta N}=0, \quad \overline{(\Delta N)^{2}}=\overline{N^{2}}-\bar{N}^{2}
$$

One may consider the expression on the left-hand side of (12.8) as quantummechanical analogue of the variance of a classical function $\alpha \alpha^{*}=\frac{1}{2 \hbar}\left(q^{2}+p^{2}\right)$ proportional to the intensity. Accepting this, in quantum optics this quantity possesses a minimum of uncertainty which cannot be undercut in contrast to classical optics where it can be equal to zero. However, one may simply consider $\overline{(\Delta N)^{2}}$ as analogue to a classical uncertainty where, however, we remind that $N=a^{\dagger} a=\frac{1}{2 \hbar}\left(Q^{2}+P^{2}-\hbar I\right)$ does not admit a limiting transition $\hbar \rightarrow 0$ to a finite classical corresponding quantity (see also remark after Eq. (2.3)).

The minimum of the left-hand side of (12.8) where the inequality makes the transition to an equality is obtained for all number states $\varrho=|n\rangle\langle n|,(n=0,1,2, \cdots)$

$$
\left\langle n\left|\mathcal{S}\left\{a^{\dagger 2} a^{2}\right\}\right| n\right\rangle-\left\langle n\left|\mathcal{S}\left\{a^{\dagger} a\right\}\right| n\right\rangle^{2}=\frac{1}{4}
$$

For coherent states $\varrho=|\alpha\rangle\langle\alpha|$ one does not obtain this minimum value on the right-hand side and find instead

$$
\left\langle\alpha\left|\mathcal{S}\left\{a^{\dagger 2} a^{2}\right\}\right| \alpha\right\rangle-\left\langle\alpha\left|\mathcal{S}\left\{a^{\dagger} a\right\}\right| \alpha\right\rangle^{2}=\alpha \alpha^{*}+\frac{1}{4}, \quad \alpha^{*} \alpha=\bar{N}
$$

For thermal states to a harmonic oscillator of frequency $\omega$ with density operator $\varrho(\bar{N})$ according to

$$
\varrho(\bar{N})=\frac{\mathrm{e}^{-x N}}{\left\langle\mathrm{e}^{-x N}\right\rangle}=\frac{1}{\bar{N}+1}\left(\frac{\bar{N}}{\bar{N}+1}\right)^{N}=\frac{1}{\bar{N}+1} \sum_{n=0}^{\infty}\left(\frac{\bar{N}}{\bar{N}+1}\right)^{n}|n\rangle\langle n|
$$

with abbreviation $x \equiv \frac{\hbar \omega}{\kappa T}$ to temperature $T$ and with $\kappa$ the Boltzmann constant and with the relations

$$
\left\langle\mathrm{e}^{-x N}\right\rangle=\sum_{n=0}^{\infty} \mathrm{e}^{-x n}=\frac{1}{1-\mathrm{e}^{-x}}, \quad \bar{N}=\frac{\mathrm{e}^{-x}}{1-\mathrm{e}^{-x}}, \quad \mathrm{e}^{-x}=\frac{\bar{N}}{\bar{N}+1}, \quad \overline{N^{2}}=\bar{N}(2 \bar{N}+1)
$$

one obtains

$$
\left\langle\varrho(\bar{N}) \mathcal{S}\left\{a^{\dagger 2} a^{2}\right\}\right\rangle-\left\langle\varrho(\bar{N}) \mathcal{S}\left\{a^{\dagger} a\right\}\right\rangle^{2}=\bar{N}(\bar{N}+1)+\frac{1}{4}
$$

Thus we have illustrated the inequality (12.8) for three important categories of states.

Instead of symmetrical (Weyl) ordering one may consider normal ordering in analogy to (12.5) with 


$$
\mathcal{N}\left\{a^{\dagger 2} a^{2}\right\}=a^{\dagger}\left(a a^{\dagger}-I\right) a=N(N-I)=\left(\mathcal{N}\left\{a^{\dagger} a\right\}\right)^{2}-N
$$

In a way which is analogous to (12.7) one derives the well-known inequality

$$
\overline{\mathcal{N}\left\{a^{\dagger 2} a^{2}\right\}}-\overline{\mathcal{N}\left\{a^{\dagger} a\right\}}{ }^{2}=\overline{(\Delta N)^{2}}-\bar{N} \geq-\bar{N}
$$

The left-hand side of this inequality may take on positive as well as negative values. It becomes equal to zero for coherent states $\varrho=|\alpha\rangle\langle\alpha|$ which obey a Poisson statistics defined in classical probability theory by the probabilities $p_{n}=\mathrm{e}^{-\mu} \frac{\mu^{n}}{n !}$ with $\mu \geq 0$ as a parameter and in quantum optics with respect to the eigenvectors $|n\rangle$ and $\langle n|$ of the operator $N$ by

$$
p_{n} \equiv\langle n \mid \alpha\rangle\langle\alpha \mid n\rangle=\exp \left(-\alpha \alpha^{*}\right) \frac{\left(\alpha \alpha^{*}\right)^{n}}{n !}=\exp (-\bar{N}) \frac{\bar{N}^{n}}{n !} \Rightarrow \overline{N^{2}}=\bar{N}^{2}+\bar{N}
$$

as it is well known. The probabilities $p_{n}$ alone do not determine the coherent states since information about the phase is absent and much less the normally ordered moments $\overline{a^{\dagger} a}=\bar{N}$ and $\overline{a^{\dagger 2} a^{2}}=\overline{N^{2}}-\bar{N}$ do this alone. In next Section we consider shortly the reconstruction of a (one-mode) state from its normally ordered moments.

If one looks to the quantity (12.16) not only as to a pure definition but as a quantity which can be measured and which, moreover, is the quantummechanical analogue of a classical quantity which last can take on only non-negative values then this becomes highly problematic.

Both quantities on the left-hand sides of (12.8) and in the middle (12.16) cannot directly be measured but can only be calculated from measured quantities of $\overline{N^{2}}$ (or $\overline{a^{\dagger 2} a^{2}}$ ) and of $\bar{N}$. Glauber in the measurement theory within his lectures [7] considered the following two cases: 1 . measurement by one-atom photon detector (chap. 4) and 2. measurement by multi-atom photon detector (chap. 5). The conclusion was that since the detectors are basically in the ground state the expectation values of powers of normally ordered annihilation and creation operators are measured. On the basis of the inequality (12.16) Mandel [29] (see also [30]) defined sub-Poissonian and super-Poissonian statistics in quantum optics in dependence on the sign of this quantity, "sub"-Poissonian if $\overline{a^{\dagger 2} a^{2}}-{\overline{a^{\dagger} a}}^{2}<0$ and "super"-Poissonian if $\overline{a^{\dagger 2} a^{2}}-{\overline{a^{\dagger} a}}^{2}>0$. With effort to the difficult task to implement the measurement theory to photon statistics [7] [8] [11] [31] Paul calculated and discussed anti-bunching of states as a typical non-classical property with no correspondence in classical optics in [32] and in [33] (anti-bunching occasionally renamed there in anti-correlations). In a short paper of Zou and Mandel [34] these authors reclaimed that Paul [32] does not consider anti-bunching and bunching but instead of this sub- and super-Poissonian statistics and that anti-bunching is not a property of a state but a property of the time evolution of a state when the time derivative of the quantity $\overline{a^{\dagger 2} a^{2}}$ becomes positive. We are not of the opinion that the notions of sub- and super-Poissonian statistics of Mandel are much better since the prefixes 
"sub" and "super" are misleading and suggest too much that the statistics of states in quantum optics can be linearly ordered with the Poisson statistics of the coherent states in the "middle". Besides the coherent states a very large category of different states possess for arbitrary given $\bar{N}$ the same expectation values $\overline{N^{2}}$ as given in (12.17) (exception: $\bar{N}=0$ which is uniquely only possible for the vacuum state $|0\rangle\langle 0|$ ). This becomes clear from the reconstruction of states by their normally ordered moments. These states can be very far from coherent states and may possess even the greatest possible distance to the nearest coherent state as can be calculated using the Hilbert-Schmidt distance, for example, for sets of some squeezed coherent states or what is the same of displaced squeezed vacuum states in the limiting procedure to maximal squeezing but with the same values $\overline{a^{\dagger} a}$ and $\overline{a^{\dagger 2} a^{2}}$ as the considered coherent states. This means that one cannot establish a linear ordering by means of the parameter (12.16) under fixed $\bar{N}$ and that one may continuously go from sub-Poissonian to super-Poissonian statistics without touching the Poisson statistics of coherent states and one cannot expect a very unique behavior of states with sub- and super-Poissonian statistics $^{5}$. The separation of sub- and super-Poissonian statistics goes amidst within the set of squeezed coherent states. Such orderings which are not a full linear ordering are called semi-orderings.

Besides the quantity (12.16) there are often used corresponding relative quantities obtained by division of (12.16) by $\bar{N}$ [30] or by $\bar{N}^{2}$ [33] where only the last corresponds to approaches in classical theory if the investigated quantity possesses a dimension. In case of the number operator $N$ such a division by the squared expectation value $\bar{N}^{2}$ enlarges without any further changes the importance of an effect for small expectation values $\bar{N}$, in particular, in this case for $\bar{N}<1$ suggesting its highly quantum character for very small expectation values $\bar{N} \ll 1$. This was estimated in [33] (in the middle of p. 187) as a pleasant agreement with Bohr's correspondence principle according to which in the limiting case of high excitations (here mean photon numbers $\bar{N}$ ) the quantum-mechanical description should make the transition to a classical one. On the other side, the smaller $\bar{N}$ the nearer the state is to the vacuum state $\varrho=|0\rangle\langle 0|$ and in the limiting case $\bar{N} \rightarrow 0$ it becomes the vacuum state. This even can be described by an inequality (Section 14) for the distance to the vacuum state which continuously is reached for $\bar{N} \rightarrow 0$. The vacuum state is a coherent state with vanishing complex displacement parameter and according to the usual opinion, the coherent states are the "most classical states".

\section{Reconstruction of Density Operator from Normally Ordered Moments}

A general quantum-mechanical state (here of the free electromagnetic field) is

${ }^{5}$ In the table 1 on p. 187 in [33] such a subdivision is made with respect to the sign of the quantity (16) but the case of its vanishing is identified with Poisson statistics and it was forgotten to mention that this does not necessarily mean the coherent states with their Poisson statistics. 
fully characterized by its density operator $\varrho$. If it is known one may determine from it, for example, the matrix element $\langle m|\varrho| n\rangle$ with the number states and $\rho=\sum_{m=0}^{\infty} \sum_{n=0}^{\infty}|m\rangle\langle m|\varrho| n\rangle\langle n|$ is already the reconstruction formula for the density operator (suppose that it is only one mode). One may also determine the moments of the density operator with powers of the annihilation and creation operators, in simplest case in normal ordering $\left\langle\varrho a^{\dagger k} a^{l}\right\rangle$ as another kind of "coordinate representation" of the density operator $\varrho$ (analogously to representation of vectors $\boldsymbol{r}$ by coordinates). The reconstruction of a density operator from its moments is more complicated than from matrix elements of the number states since it corresponds to a non-orthogonal basis system.

The reconstruction formula of the density operator $\varrho$ from its normally ordered moments $\left\langle\varrho a^{\dagger k} a^{l}\right\rangle$ was derived in [35] with the result (is correct also for arbitrary operators $A$ if involved quantities exist)

$$
\varrho=\sum_{k=0}^{\infty} \sum_{l=0}^{\infty}\left\langle\varrho a^{\dagger k} a^{l}\right\rangle a_{k, l}=\sum_{k=0}^{\infty} \sum_{l=0}^{\infty}\left\langle\varrho a_{k, l}\right\rangle a^{\dagger k} a^{l}
$$

where $a_{k, l}$ is the abbreviation for a set of auxiliary operators necessary for the reconstruction and defined as follows (remind that $\langle A\rangle$ means the trace of an operator $A$ and $|l-j\rangle$ and $\langle k-j|$ are number states)

$$
\begin{aligned}
a_{k, l} & \equiv \sum_{j=0}^{\{k, l\}} \frac{(-1)^{j}|l-j\rangle\langle k-j|}{j ! \sqrt{(k-j) !(l-j) !}} \\
& =\frac{1}{k ! ! !} \sum_{i=-\{k, l\}}^{\infty} \frac{(-1)^{i}(k+l+i) !}{(k+i) !(l+i) !} a^{\dagger l+i} a^{k+i}, \quad a_{0,0}=|0\rangle\langle 0|
\end{aligned}
$$

The relation

$$
\left\langle a_{k, l} a^{\dagger m} a^{n}\right\rangle=\sum_{j=0}^{\{k, l\}} \frac{(-1)^{j}\left\langle k-j\left|a^{\dagger m} a^{n}\right| l-j\right\rangle}{j ! \sqrt{(k-j) !(l-j) !}}=\delta_{k, m} \delta_{l, n}
$$

shows that the two sets of "coordinates" $\left\langle\varrho a_{k, l}\right\rangle$ and $\left\langle\varrho a^{\dagger k} a^{l}\right\rangle$ are related to each other similarly as covariant and contravariant components of a vector. From (13.3) follows as special case $m=n=0$ for the traces of the operators $a_{k, l}$

$$
\left\langle a_{k, l}\right\rangle=\delta_{k, 0} \delta_{k, l}=\delta_{k, 0} \delta_{l, 0}
$$

and using this together with (13.1) one may check the normalization

$$
\langle\varrho\rangle=\sum_{k=0}^{\infty} \sum_{l=0}^{\infty}\left\langle\varrho a^{\dagger k} a^{l}\right\rangle\left\langle a_{k, l}\right\rangle=1
$$

In contrast, the traces of $a^{\dagger k} a^{l}$ are also vanishing for $k \neq l$ but do not possess finite values for $k=l$.

Usually it is assumed that a density operator $\varrho$ expresses the maximum knowledge for an ensemble of states which individual members are in states described by different exact wave functions that means by averaging over density operators for pure states $\varrho_{i}=\left|\psi_{i}\right\rangle\left\langle\psi_{i}\right|$ with probability $p_{i}$ as coefficients in front of them, i.e. $\varrho=\sum_{i} p_{i}\left|\psi_{i}\right\rangle\left\langle\psi_{i}\right|,\left(\sum_{i} p_{i}=1\right)$. Then arise problems of 
determination of the possible pure states $\left|\psi_{i}\right\rangle\left\langle\psi_{i}\right|$ together with a discrete averaging function $p_{i} \geq 0$ leading to the diagonal form of the density operator.

For a full reconstruction of the density operator $\varrho$ according to the basic Formulas (13.1) we need all normally ordered moments $\overline{a^{\dagger k} a^{l}} \equiv\left\langle\varrho a^{\dagger k} a^{l}\right\rangle$. If one determines only the diagonal values $\overline{a^{\dagger k} a^{k}},(k=0,1,2, \cdots)$ as in photon statistics then one can reconstruct only a part $\varrho_{0}$ of the density operator $\varrho$ of the form

$$
\varrho_{0}=\sum_{k=0}^{\infty} \overline{a^{\dagger k} a^{k}} a_{k, k}=\sum_{k=0}^{\infty} \overline{\frac{N !}{(N-k I) !}} \sum_{j=0}^{k} \frac{(-1)^{j}|k-j\rangle\langle k-j|}{j !(k-j) !}, \quad\left\langle\varrho_{0}\right\rangle=1
$$

where the factorial moments $\overline{a^{\dagger k} a^{k}}$ are connected with the expectation values $\overline{N^{l}}$ of the number operator $N$ together with its inversion by

$$
\overline{\frac{N !}{(N-k I) !}}=\sum_{l=0}^{k} s(k, l) \overline{N^{l}}, \quad \overline{N^{k}}=\sum_{l=0}^{k} s(k, l) \overline{\frac{N !}{(N-l I) !}}
$$

with $s(k, l)$ and $S(k, l)$ the Stirling numbers of first and second kind, respectively. Writing down explicitly the first four sum terms of (13.6) we have

$$
\begin{aligned}
\varrho_{0}= & |0\rangle\langle 0|+\bar{N}\left(\frac{|1\rangle\langle 1|}{0 ! 1 !}-\frac{|0\rangle\langle 0|}{1 ! 0 !}\right)+\left(\overline{N^{2}}-\bar{N}\right)\left(\frac{|2\rangle\langle 2|}{0 ! 2 !}-\frac{|1\rangle\langle 1|}{1 ! 1 !}+\frac{|0\rangle\langle 0|}{2 ! 0 !}\right) \\
& +\left(\overline{N^{3}}-3 \overline{N^{2}}+2 \bar{N}\right)\left(\frac{|3\rangle\langle 3|}{0 ! 3 !}-\frac{|2\rangle\langle 2|}{1 ! 2 !}+\frac{|1\rangle\langle 1|}{2 ! 1 !}-\frac{|0\rangle\langle 0|}{3 ! 0 !}\right)+\cdots
\end{aligned}
$$

and we see that it is absolutely insufficient to conclude that in case of $\overline{N^{2}}-\bar{N}=\bar{N}^{2}$ we have a Poisson distribution to density operator $\varrho_{0}$

$$
\varrho_{0}=\exp (-\bar{N}) \sum_{n=0}^{\infty} \frac{\bar{N}^{n}}{n !}|n\rangle\langle n|, \quad \overline{\frac{N !}{(N-k I) !}}=\bar{N}^{k}, \quad \overline{N^{k}}=\sum_{l=0}^{k} S(k, l) \bar{N}^{l}
$$

in particular, for high expectation values $\bar{N}$, apart from the full absence of information about the phases (expectation values $\overline{a^{\dagger k} a^{l}}$ with $k \neq l$ ). For a "sufficient" reconstruction of a density operator $\varrho_{0}$ we need, at least, expectation values $\overline{N^{l}}$ up to values $l \rightarrow n \gg \bar{N}$ [35]. For $\bar{N} \ll 1$ the density operator $\varrho_{0}$ is near to that for the vacuum state but this is better to see from the Hilbert-Schmidt distance to the vacuum state (next Section).

The treatment of reconstruction of the density operator reveals many interesting problems partially not solved up to now. Within which limits can a density operator reconstructed with incomplete knowledge of necessary quantities (expectation values, moments) if only a small part of them is known or if it is assumed that the density operator possesses only one, two, three and so far eigenvalues different from zero. If only one eigenvalue is different from zero (then equal to 1) we have the problem of reconstruction of pure states. Also in this case one has an arbitrariness, for example, if $\bar{N}$ and $\overline{(\Delta N)^{2}}-\bar{N}=0$ is known which admits as well as coherent states as different squeezed states and also other states. We do not consider this here (some elements of treatment of squeezed states we developed already in former publications, e.g., [36]). 


\section{Hilbert-Schmidt Distance of Two Quantum-Mechanical States}

A measure how near or far are two quantum-mechanical states is their distance. Distances are determined by a few axioms. They have to be non-negative and should be zero for equal states and they should obey the triangular inequality (to find in almost every monograph about functional analysis). From the many possible distances the only distance in quantum theory with which one can calculate in convenient way is the Hilbert-Schmidt distance determined by the Hilbert-Schmidt norm of the elements of the Hilbert space of states. Since a general state is described by a density operator this is in our case the Hilbert space of all (normalizable) operators $A, B, \cdots$ according to their scalar products $\langle A B\rangle$. Thus the Hilbert-Schmidt distance of two states described by the density operators $\varrho$ and $\varrho^{\prime}$ and their special cases of pure states $\varrho=|\psi\rangle\langle\psi|$ or (and) $\varrho^{\prime}=\left|\psi^{\prime}\right\rangle\left\langle\psi^{\prime}\right|$ we define the distances denoted by $\| \ldots . \mid$ [37] [38]

$$
\begin{aligned}
& \left\|\varrho-\varrho^{\prime}\right\| \equiv \sqrt{\left\langle\left(\varrho-\varrho^{\prime}\right)^{2}\right\rangle}=\sqrt{\left\langle\varrho^{2}\right\rangle+\left\langle\varrho^{\prime 2}\right\rangle-2\left\langle\varrho \varrho^{\prime}\right\rangle} \\
& \| \varrho-\left|\psi^{\prime}\right\rangle\left\langle\psi^{\prime}\right| \| \equiv \sqrt{\left\langle\left(\varrho-\left|\psi^{\prime}\right\rangle\left\langle\psi^{\prime}\right|\right)^{2}\right\rangle}=\sqrt{\left\langle\varrho^{2}\right\rangle+1-2\left\langle\psi^{\prime}|\varrho| \psi^{\prime}\right\rangle} \\
& \| \psi\rangle\left\langle\psi|-| \psi^{\prime}\right\rangle\left\langle\psi^{\prime}\right| \| \equiv \sqrt{\left\langle\left(|\psi\rangle\left\langle\psi|-| \psi^{\prime}\right\rangle\left\langle\psi^{\prime}\right|\right)^{2}\right\rangle}=\sqrt{2\left(1-\left\langle\psi^{\prime} \mid \psi\right\rangle\left\langle\psi \mid \psi^{\prime}\right\rangle\right)}
\end{aligned}
$$

The possible phases of abstract pure states described by $|\psi\rangle$ do not play a role in these definitions but the normalization $\langle\psi \mid \psi\rangle=1$ does. The maximum difference between two normalized pure states is equal to $\sqrt{2}$ and happens for mutually orthogonal states that is the same as for orthogonal unit vectors in a vector space. We mention that the factor $\sigma \equiv\langle\varrho\rangle^{2}-\left\langle\varrho^{2}\right\rangle=1-\left\langle\varrho^{2}\right\rangle$ is often called the impurity factor of a state described by density operators $\varrho$. For impurity factors $\sigma \neq 0$ the maximum possible difference between such states is smaller than $\sqrt{2}$ in dependence on $\sigma$.

Results derived in quantum optics for coherent states are often most near to corresponding results from classical optics and are in this sense the most "classical" states. Therefore, it is natural to define the distance $d(\varrho,|\alpha\rangle\langle\alpha|)$ of a state $\varrho$ to the nearest coherent state $|\alpha\rangle\langle\alpha|$ as a measure of nonclassicality ${ }^{6}$

$$
d_{\text {noncl }}(\varrho) \equiv \operatorname{Min}_{\alpha \in \mathbb{C}} \| \varrho-|\alpha\rangle\langle\alpha| \|=\operatorname{Min}_{\alpha \in \mathbb{C}} \sqrt{\left\langle\varrho^{2}\right\rangle+1-2\langle\alpha|\varrho| \alpha\rangle}
$$

One may modify this definition for mixed states by substituting the class of all coherent states by the class of all displaced Gaussian states (displaced thermal states) with the same impurity factor $\sigma=1-\left\langle\varrho^{2}\right\rangle$ as the considered state if one knows its impurity factor [38].

For the distance of a pure state $|\psi\rangle=\sum_{n=0}^{\infty} c_{n}|n\rangle$ to the vacuum state $|0\rangle$ one finds the inequality

${ }^{6}$ We did not very emphasize this in [37] since at this time we did not want to come in conflict with other existing definitions and categories of nonclassicality, in particular, with anti-bunching [32]. 


$$
\begin{aligned}
\||\psi\rangle\langle\psi|,| 0\rangle\langle 0| \| & \equiv \sqrt{2(1-\langle 0 \mid \psi\rangle\langle\psi \mid 0\rangle)}=\sqrt{2\left(1-c_{0} c_{0}^{*}\right)}=\sqrt{2 \sum_{n=1}^{\infty} c_{n} c_{n}^{*}} \\
& \leq \sqrt{2 \sum_{n=1}^{\infty} n c_{n} c_{n}^{*}}=\sqrt{2 \bar{N}}, \quad(\bar{N}<1)
\end{aligned}
$$

Since the maximum possible distance of two normalized pure states in Hilbert space is $\sqrt{2}$ this inequality becomes trivial for $\bar{N} \geq 1$. Thus for $\bar{N}<1$ the distance to the vacuum state goes for $\bar{N} \rightarrow 0$ at least with $\sqrt{2 \bar{N}} \rightarrow 0$ against zero. This means that for $\bar{N} \ll 1$ the absolute nonclassical effects for sub-Poissonian states cannot very much differ from that for the vacuum state. Between the measure of nonclassicality of states by their smallest possible distance to a certain coherent state and the negativity of the measure (12.16) it is difficult to find some correlation.

\section{Conclusions}

In present article we have investigated in some detail the classical to quantum correspondence in the sense of Weyl for an important class of classical phase-space functions and quantum-mechanical operators and derived different representations, mainly representations by number states. The coefficients in these representations could be written in compact form using the Jacobi polynomials $\mathrm{P}_{n}^{(\alpha, \beta)}(u)$. It was astonishing how all the considered important special cases could be obtained by specializing the Jacobi polynomials in corresponding way and how they provided in some cases different more simple formulae for the even and odd cases of the main indices but which could not be joined on a lower level than on the level of the Jacobi polynomials. One may state that the considered problem leads to one of the most important applications of the Jacobi polynomials showing their magnificence.

Despite the somehow beautiful application of the Jacobi polynomials to the Weyl correspondence this is not fully satisfying because it is not clear how the expectation values of large categories of calculated operators may be measured in experiments. It is also not clear in which or whether or not in all situations the most aesthetical symmetrical ordering of functions of the canonical operators is the right one realized by fundamental quantum processes. In the theory of Glauber [7] for measurements of the photon statistics there are only considered the two cases of devices for measurement of photon numbers with one atom and with many atoms.

In particular, the phase measurements are problematic since in interaction with classical devices the amplitude and phase is difficult to separate and the interaction operators are involved only as the full annihilation and creation operators without separation of the phase. For a long time up to the nineties it was common sense that classical action and angle variable should become quantum-mechanical Hermitean operators that is problematic for the angle operators for which the measured values are determined only up to a multiple of $2 \pi$. For a single measured value of the phase one may say that it is undetermined 
up to additional multiple value of $2 \pi$. However, already for two measured values it is then difficult to determine what is its mean value. This difficulty can be avoided as we did if one considers only periodic functions of the phase.

Finally, it should be difficult for the same reason to generate "good" states with well determined phase for interference experiments and to distinguish experimentally between the quantum-mechanical approaches to the phase in the formalisms of Susskind and Glogower and in the Weyl correspondence by the symmetrical ordering of annihilation and creation operators applied to the phase.

\section{References}

[1] Weyl, H. (1927) Quantenmechanik und Gruppentheorie. Zeitschrift für Physik, 46, 1-46. https://doi.org/10.1007/BF02055756

[2] Weyl, H. (1928) Gruppentheorie und Quantenmechamik. Hirzel, Leipzig. (English translation: The Theory of Groups and Quantum Mechanics, Dover, New York 1931, I used the Russian translation of the English edition from 1931 from Publishing House Nauka, Moskva 1986 (Editor D.P. Shelobeko)).

[3] Wigner, E. (1932) On the Quantum Correction For Thermodynamic Equilibrium. Physical Review, 40, 749. (Republished in [4])

https://doi.org/10.1103/PhysRev.40.749

[4] Kim, Y.S. and Noz, M.E. (1991) Phase Space Picture of Quantum Mechanics. World Scientific, Singapore. (With Republication of Article [3]).

[5] Vourdas, A. (2004) Quantum Systems with Finite Hilbert Space. Reports on Progress in Physics, 67, 267-320, https://doi.org/10.1088/0034-4885/67/3/R03

[6] Glauber, R.J. (1963) Coherent and Incoherent States of the Radiation Field. Physical Review, 131, 2766. (Republished in [9]) https://doi.org/10.1103/PhysRev.131.2766

[7] Glauber, R.J. (1965) Optical Coherence and Photon Statistics. In: De Witt, C., Blandin, A. and Cohen-Tannoudji, C., Eds., Quantum Optics and Electronics, Gordon and Breach, New York, p. 144. (I used the Russian translation of this book, Mir, Moskva 1966.)

[8] Klauder, J.R. and Sudarshan, E.C.G. (1968) Fundamentals of Quantum Optics. W.A. Benjamin, Inc., New York. (Russian Translation, Mir, Moskva 1970)

[9] Klauder, J.R. and Skagerstam, B.-S. (1985) Coherent States. World Scientific, Singapore. https://doi.org/10.1142/0096

[10] Wünsche, A. (1996) The Complete Gaussian Class of Quasiprobabilities and Its Relation to Squeezed States and Their Excitations. Quantum and Semiclassical Optics, 8, 343-379. https://doi.org/10.1088/1355-5111/8/2/003

[11] Peřina, J. (1971) Coherence of Light. Van Nostrand Reinhold Company, New York.

[12] Peřinová, V., Lukš, A. and Peřina, J. (1998) Phase in Optics. World Scientific, Singapore. https://doi.org/10.1142/3541

[13] Wünsche, A. (2015) Quantum-Mechanical Cumulant Expansions and Their Application to Phase-Space and to Phase Distributions. Physica Scripta, 90, 074063. https://doi.org/10.1088/0031-8949/90/7/074063

[14] Garraway, B.M. and Knight, P.L. (1992) Quantum Phase Distributions and Quasidistributions. Physical Review A, 46, R5346.

[15] Wünsche, A. (1995) Ordered Operator Expansions and Reconstruction from Or- 
dered Moments. Journal of Optics B-Quantum and Semiclassical Optics, 1, 264-288. https://doi.org/10.1088/1464-4266/1/2/010

[16] Louisell, W.H. (1973) Quantum Statistical Properties of Radiation. John Wiley \& Sons, New York.

[17] Wünsche, A. (2015) Generating Functions for Products of Laguerre 2D and Hermite 2D Polynomials. 6, 2142-2168.

[18] Wünsche, A. (2000) General Hermite and Laguerre Two-Dimensional Polynomials, Journal of Physics A: Mathematical and General, 33, 1603-1629. (Corrigendum Journal of Physics A: Mathematical and General, 33, 3531 (2000)) https://doi.org/10.1088/0305-4470/33/8/307

[19] Szegö, G. (1959) Orthogonal Polynomials (Rev. Ed.). American Mathematical Society, New York.

[20] Courant, R. and Hilbert, D. (1993) Methoden der mathematischen Physik (4. Aufl.). Springer-Verlag, Berlin. (2nd Edition in two vol. 1930 and 1937, Engl. transl.: John-Wiley-Interscience 1953 and 1962)

[21] Erdélyi, A. (1953) Higher Transcendental Functions, Vol. II. McGraw-Hill, New York. (Bateman Manuscript Project; Its Russian translation which I use adds H. Bateman to the author names in the three volumes giving credit in this way to his great contribution in the Project: Nauka, Moskva 1974 (Editor N.Ya. Vilenkin)).

[22] Koornwinder, T.H., Wong, R., Koekoek, R. and Swarttouw, R.F. (2010) Orthogonal Polynomials. In: Olver, F.W.J., Lozier, D.W., Boisvert, R.F. and Clark, Ch.W., Eds., NIST Hamdbook of Mathematical Functions, Cambridge University Press, Cambridge, chap. 18, 435-484.

[23] Olver, F.W.J., Lozier, D.W., Boisvert, R.F. and Clark, Ch.W. (2010) NIST Handbook of Mathematical Functions. Cambridge University Press, Cambridge.

[24] Wünsche, A. (2017) Operator Methods and SU(1,1) Symmetry in the Theory of Jacobi and of Ultraspherical Polynomials. Applied Mathematics, 7, 213-261. https://doi.org/10.4236/apm.2017.72012

[25] Riordan, J. (1958) An Introduction to Combinatorial Analysis. John Wiley, New York.

[26] van Lint, J.H. and Wilson, R.M. (1992) A Course in Combinatorics. Cambridge University Press, Cambridge.

[27] Susskind, L. and Glogower, J. (1964) Quantum Mechanical Phase and Time Operator. Physics, 1, 49-61.

[28] Carruthers, P. and Nieto, M. (1968) Phase and Angle Variables in Quantum Mechanics. Reviews of Modern Physics, 40, 411. (Republished in: Coherent States in Quantum Theory (in Russian), p. 71, Editor V.I. Man'ko, Mir, Moskva 1972). https://doi.org/10.1103/RevModPhys.40.411

[29] Mandel, L. (1979) Sub-Poissonian Photon Statistics in Resonance Fluorescence. Optics Letters, 4, 205-207. https://doi.org/10.1364/OL.4.000205

[30] Mandel, L. and Wold, E. (1995) Optical Coherence and Quantum Optics. Cambridge. https://doi.org/10.1017/CBO9781139644105

[31] Leonhardt, U. (1997) Measuring the Quantum State of Light. Cambridge University Press, Cambridge.

[32] Paul, H. (1982) Photon Antibunching. Reviews of Modern Physics, 54, 1061. https://doi.org/10.1103/RevModPhys.54.1061

[33] Paul, H. (1995) Photonen. B.G. Teubner, Stuttgart. (In German) https://doi.org/10.1007/978-3-322-96700-8 
[34] Zou, X.T. and Mandel, L. (1990) Photon-Antibunching and Sub-Poissonian Photon Statistics. Physical Review A, 41, 475. https://doi.org/10.1103/PhysRevA.41.475

[35] Wünsche, A. (1990) Reconstruction of Operators from Their Normally Ordered Moments for a Single Boson Mode. Quantum Optics, 2, 453-466. https://doi.org/10.1088/0954-8998/2/6/004

[36] Wünsche, A. (2003) Squeezed States. In: Dodonov, V.V. and Man'ko, V.I., Eds., Theory of Nonclassical States of Light, Taylor and Francis, London and New York. 95-152.

[37] Wünsche, A. (1995) The Distance to Poissonian Statistics as a Supplementary Measure in Quantum Optics. Applied Physics B, 60, 119-122.

[38] Dodonov, V.V., Man'ko, O.V., Man'ko, V.I. and Wünsche, A. (2000) Hilbert-Schmidt Distance and Nonclassicality of States in Quantum Optics. Journal of Modern Optics, 47, 633-654.

[39] Gradshteyn, I.S. and Ryzhik, I.M. (1963) Tables of Series, Products and Integrals. 4th Edition, Nauka, Moscow. (Many translation into other languages and later editions) 


\section{Appendix A}

Transition from power functions to symmetrized operators and a few relations for Jacobi polynomials

We now investigate the case of transition from the product of a power function $\left(\sqrt{\alpha \alpha^{*}}\right)^{k}$ with a phase function $\left(\sqrt{\frac{\alpha}{\alpha^{*}}}\right)^{l}$ into a symmetrized quantum-mechanical operator in the sense of Weyl. According to the general scheme we find applying relation (5.14) with intermediate transition to polar coordinate $\left(|\alpha|, \mathrm{e}^{\mathrm{i} \varphi}\right)=\left(\sqrt{\alpha \alpha^{*}}, \sqrt{\frac{\alpha}{\alpha^{*}}}\right)$

$$
A^{(k, l)} \equiv \mathcal{S}\left\{\left(\sqrt{a a^{\dagger}}\right)^{k}\left(\sqrt{\frac{a}{a^{\dagger}}}\right)^{l}\right\}
$$$$
=\frac{2}{\pi} \int \frac{\mathrm{i}}{2} \mathrm{~d} \alpha \wedge \mathrm{d} \alpha^{*}\left(\sqrt{\alpha \alpha^{*}}\right)^{k}\left(\sqrt{\frac{\alpha}{\alpha^{*}}}\right)^{l} \exp \left(-2 \alpha \alpha^{*}\right) \sum_{m=0}^{\infty} \sum_{n=0}^{\infty} \frac{|m\rangle\langle n|}{\sqrt{m ! n !}} \mathrm{L}_{m, n}\left(2 \alpha, 2 \alpha^{*}\right)
$$$$
=\sum_{m=0}^{\infty} \sum_{n=0}^{\infty} \frac{|m\rangle\langle n|}{\sqrt{m ! n !}} \sum_{j=0}^{\{m, n\}} \frac{(-1)^{j} m ! n !}{j !(m-j) !(n-j) !} 2^{m+n-2 j}
$$$$
\cdot \frac{2}{\pi} \int_{0}^{+\infty} \mathrm{d}|\alpha||\alpha| \exp \left(-2|\alpha|^{2}\right)|\alpha|^{m+n+k-2 j} \underbrace{\int_{0}^{2 \pi} \mathrm{d} \varphi \mathrm{e}^{\mathrm{i}(m+l-n) \varphi}}_{=2 \pi \delta_{m+1, n}}
$$

$=\sum_{m=0}^{\infty} \frac{|m\rangle\langle m+l|}{\sqrt{m !(m+l) !}} \sum_{j=0}^{m} \frac{(-1)^{j} m !(m+l) !}{j !(m-j) !(m+l-j) !} 2^{2 m+l-2 j} 4 \int_{0}^{+\infty} \mathrm{d}|\alpha| \mathrm{e}^{-2|\alpha|^{2}}|\alpha|^{2 m+k+l-2 j+1}$

$=\sum_{m=0}^{\infty} \frac{|m\rangle\langle m+l|}{\sqrt{m ! m+l !}} \sum_{j=0}^{m} \frac{(-1)^{j} m !(m+l) !}{j !(m-j) !(m+l-j) !} 2^{2 m+l-2 j} \frac{\left(m+\frac{k+l}{2}-j\right) !}{2^{m+\frac{k+l}{2}-j}}$

This can be represented in the following preliminary explicit form

$$
A^{(k, l)}=\sum_{m=0}^{\infty}|m\rangle\langle m+l| \sqrt{m !(m+l) ! 2^{-\frac{k-l}{2}}} \sum_{j=0}^{m} \frac{(-1)^{j}\left(m+\frac{k+l}{2}-j\right) !}{j !(m-j) !(m+l-j) !} 2^{m-j}
$$

We used here the explicit expression (5.3) for the Laguerre 2D polynomials $\mathrm{L}_{m, n}\left(z, z^{*}\right)$ [17].

The result (A.2) can be expressed using the Jacobi polynomials $\mathrm{P}_{n}^{(\alpha, \beta)}(u)$ or the corresponding Hypergeometric function ${ }_{2} \mathrm{~F}_{1}(a, b ; c ; z)$ in the forms (e.g., [21] (chap. 10) and [19])

$$
\begin{aligned}
\mathrm{P}_{n}^{(\alpha, \beta)}(u) & =\frac{(n+\alpha) !}{n ! \alpha !}{ }_{2} \mathrm{~F}_{1}\left(-n, n+\alpha+\beta+1 ; 1+\alpha ; \frac{1-u}{2}\right) \\
& =\left(\frac{u+1}{2}\right)^{n} \sum_{j=0}^{n} \frac{(n+\alpha) !(n+\beta) !}{j !(n-j) !(n+\alpha-j) !(j+\beta) !}\left(\frac{u-1}{u+1}\right)^{n-j} \\
& =\frac{(n+\beta) !}{(n+\alpha+\beta) !} \sum_{j=0}^{n} \frac{(-1)^{j}(2 n+\alpha+\beta-j) !}{j !(n-j) !(n+\beta-j) !}\left(\frac{u+1}{2}\right)^{n-j} \\
& =\frac{(n+\alpha) !}{n ! \alpha !}\left(\frac{1+u}{2}\right)^{n}{ }_{2} \mathrm{~F}_{1}\left(-n,-n-\beta+1 ; 1+\alpha ;-\frac{1-u}{1+u}\right)
\end{aligned}
$$


with the given known transformation relation for the Hypergeometric function leading to the relation [19] (chap. IV, Equation (4.22.1); we changed its representation slightly) ${ }^{7}$

$$
\mathrm{P}_{n}^{(\alpha, \beta)}(u)=\left(\frac{1+u}{2}\right)^{n} \mathrm{P}_{n}^{(\alpha,-2 n-\alpha-\beta-1)}\left(\frac{3-u}{1+u}\right)
$$

with changing the argument of the Jacobi polynomials and an upper index and with the symmetry relation

$$
\mathrm{P}_{n}^{(\alpha, \beta)}(u)=(-1)^{n} \mathrm{P}_{n}^{(\beta, \alpha)}(-u)
$$

For argument $u=1$ and $u=-1$ the Jacobi polynomials take on the values

$$
\begin{gathered}
\mathrm{P}_{n}^{(\alpha, \beta)}(1)=\mathrm{P}_{n}^{(\alpha,-2 n-\alpha-\beta-1)}(1)=\frac{(n+\alpha) !}{n ! \alpha !} \\
\mathrm{P}_{n}^{(\alpha, \beta)}(-1)=\mathrm{P}_{n}^{(-2 n-\alpha-\beta-1, \beta)}(-1)=(-1)^{n} \frac{(n+\beta) !}{n ! \beta !}
\end{gathered}
$$

This follows from the relations (A.3), (A.4) and (A.5). We apply this in the next section.

Using the explicit representations of the Jacobi polynomials in the third line of (A.3) and then the relation (A.4) with argument transformation from (A.2) follows

$$
\begin{aligned}
A^{(k, l)} & =\left(\frac{k+l}{2}\right) ! \sum_{m=0}^{\infty} \sqrt{\frac{m !}{(m+l) !}} 2^{-\frac{k-l}{2}} \mathrm{P}_{m}^{\left(-m+\frac{k-l}{2}, l\right)}(3)|m\rangle\langle m+l| \\
& =\left(\frac{k+l}{2}\right) ! \sum_{m=0}^{\infty} \sqrt{\frac{m !}{(m+l) !}} 2^{m-\frac{k-l}{2}} \mathrm{P}_{m}^{\left(-m+\frac{k-l}{2},-m-\frac{k+l}{2}-1\right)}(0)|m\rangle\langle m+l|
\end{aligned}
$$

Using the following general transformation relation for integer $l$ which results from a general transformation relation for the Hypergeometric function (e.g., [39], chap. 9.13, Equation 9.131) which changes the lower index of the Jacobi polynomials ${ }^{8}$

$$
\mathrm{P}_{n}^{(\alpha, l)}(u)=\frac{(n+\alpha) !(n+l) !}{n !(n+\alpha+l) !}\left(\frac{2}{1+u}\right)^{l} \mathrm{P}_{n+l}^{(\alpha,-l)}(u), \quad(l=-n,-n+1, \cdots, 0,1,2, \cdots)
$$

one may represent (A.7) also in the form

$$
\begin{aligned}
A^{(k, l)} & =\left(\frac{k-l}{2}\right) ! \sum_{m=0}^{\infty} \sqrt{\frac{(m+l) !}{m !}} 2^{-\frac{k+l}{2}} \mathrm{P}_{m+l}^{\left(-m+\frac{k-l}{2},-l\right)}(3)|m\rangle\langle m+l| \\
& =\left(\frac{k-l}{2}\right) ! \sum_{m=0}^{\infty} \sqrt{\frac{(m+l) !}{m !}} 2^{m-\frac{k-l}{2}} \mathrm{P}_{m+l}^{\left(-m+\frac{k-l}{2},-m-\frac{k+l}{2}-1\right)}(0)|m\rangle\langle m+l|
\end{aligned}
$$

In Section 10, we need the Jacobi polynomials of special argument $\mathrm{P}_{m}^{(-m-\alpha, 2 \alpha)}(3)$ or, alternatively, $\mathrm{P}_{m}^{(-m-\alpha,-m-\alpha-1)}(0)$ which for arbitrary $\alpha$ can be expressed distinguishing the even case $m=2 n$ and the odd case $m=2 n+1$ in ${ }^{7}$ This transformation possesses involutory character. Applying it to the right-hand side it leads back to the primary form $\mathrm{P}_{n}^{(\alpha, \beta)}(u)$.

${ }^{8}$ These transformations possess also involutory character such as (4). 
the following way (see also (A.4))

$$
\begin{gathered}
\mathrm{P}_{2 n}^{(-2 n-\alpha, 2 \alpha)}(3)=2^{2 n} \mathrm{P}_{2 n}^{(-2 n-\alpha,-2 n-\alpha-1)}(0)=\frac{(n+\alpha) !}{n ! \alpha !} \\
\mathrm{P}_{2 n+1}^{(-2 n-1-\alpha, 2 \alpha)}(3)=2^{2 n+1} \mathrm{P}_{2 n+1}^{(-2 n-1-\alpha,-2 n-1-\alpha-1)}(0)=\frac{(n+\alpha) !}{n ! \alpha !}
\end{gathered}
$$

The equality of the right-hand sides of these relations is a very curious fact.

\section{Appendix B}

Calculation of expressions for the transition from a smoothed classical function to a corresponding quantum-mechanical operator

We calculate in this Appendix the transition from a smoothed classical function of the canonical variables in representation by the complex variables $\left(\alpha, \alpha^{*}\right)$ to a quantum-mechanical operator in the sense of Weyl. The smoothing of the classical function $A\left(\alpha, \alpha^{*}\right)$ is made by convolution with a normalized Gaussian function as follows

$$
A_{r}\left(\alpha, \alpha^{*}\right)=\exp \left(\frac{r}{2} \frac{\partial^{2}}{\partial \alpha \partial \alpha^{*}}\right) A\left(\alpha, \alpha^{*}\right)=\frac{2}{\pi r} \exp \left(-\frac{2 \alpha \alpha^{*}}{r}\right) * A\left(\alpha, \alpha^{*}\right)
$$

where “*” denotes the convolution. According to (5.14) one has now to calculate

$$
\begin{aligned}
A_{r}= & \frac{2}{\pi} \int \frac{\mathrm{i}}{2} \mathrm{~d} \alpha \wedge \mathrm{d} \alpha^{*}\left\{\exp \left(\frac{r}{2} \frac{\partial^{2}}{\partial \alpha \partial \alpha^{*}}\right) A\left(\alpha, \alpha^{*}\right)\right\} \\
& \times \exp \left(-2 \alpha \alpha^{*}\right) \sum_{m=0}^{\infty} \sum_{n=0}^{\infty} \mathrm{L}_{m, n}\left(2 \alpha, 2 \alpha^{*}\right) \frac{|m\rangle\langle n|}{\sqrt{m ! n !}} \\
= & \frac{2}{\pi} \int \frac{\mathrm{i}}{2} \mathrm{~d} \alpha \wedge \mathrm{d} \alpha^{*} A\left(\alpha, \alpha^{*}\right)\left\{\exp \left(\frac{r}{2} \frac{\partial^{2}}{\partial \alpha \partial \alpha^{*}}\right)\right. \\
& \left.\times \exp \left(-2 \alpha \alpha^{*}\right) \sum_{m=0}^{\infty} \sum_{n=0}^{\infty} \mathrm{L}_{m, n}\left(2 \alpha, 2 \alpha^{*}\right) \frac{|m\rangle\langle n|}{\sqrt{m ! n !}}\right\}
\end{aligned}
$$

where we applied partial integration. According to Formula $A(35)$ in [17] specialized to our case we have the operator identity

$$
\begin{aligned}
& \exp \left(\frac{r}{2} \frac{\partial^{2}}{\partial \alpha \partial \alpha^{*}}\right) \exp \left(-2 \alpha \alpha^{*}\right) \\
& =\frac{1}{1+r} \exp \left(-\frac{2 \alpha \alpha^{*}}{1+r}\right) \exp \left(\frac{r(1+r)}{2} \frac{\partial^{2}}{\partial \alpha \partial \alpha^{*}}\right)\left(\frac{1}{1+r}\right)^{\alpha \frac{\partial}{\partial \alpha}+\alpha^{*} \frac{\partial}{\partial \alpha^{*}}}
\end{aligned}
$$

which applied to $\mathrm{L}_{m, n}\left(2 \alpha, 2 \alpha^{*}\right)$ in (B.2) provides

$$
\begin{aligned}
& \exp \left(\frac{r}{2} \frac{\partial^{2}}{\partial \alpha \partial \alpha^{*}}\right) \exp \left(-2 \alpha \alpha^{*}\right) \mathrm{L}_{m, n}\left(2 \alpha, 2 \alpha^{*}\right) \\
& =\frac{1}{1+r} \exp \left(-\frac{2 \alpha \alpha^{*}}{1+r}\right) \exp \left(\frac{r(1+r)}{2} \frac{\partial^{2}}{\partial \alpha \partial \alpha^{*}}\right) \mathrm{L}_{m, n}\left(\frac{2 \alpha}{1+r}, \frac{2 \alpha^{*}}{1+r}\right) \\
& =\frac{1}{1+r} \exp \left(-\frac{2 \alpha \alpha^{*}}{1+r}\right)\left(\sqrt{\frac{1-r}{1+r}}\right)^{m+n} \mathrm{~L}_{m, n}\left(\frac{2 \alpha}{\sqrt{1-r^{2}}}, \frac{2 \alpha^{*}}{\sqrt{1-r^{2}}}\right)
\end{aligned}
$$


We applied here first that $\lambda^{\alpha \frac{\partial}{\partial \alpha}}$ is the multiplication operator of the variable $\alpha$ of a function $f\left(\alpha, \alpha^{*}\right)$ by $\lambda$ that means it makes the transition $f\left(\alpha, \alpha^{*}\right) \rightarrow f\left(\lambda \alpha, \alpha^{*}\right)$. The second transformation can be obtained if we rewrite the definition (5.1) of the Laguerre 2D polynomials for $\mathrm{L}_{m, n}\left(\frac{z}{b}, \frac{z^{*}}{b}\right)$ with scalar parameter $b$

$$
\mathrm{L}_{m, n}\left(\frac{z}{b}, \frac{z^{*}}{b}\right)=\exp \left(-b^{2} \frac{\partial^{2}}{\partial z \partial z^{*}}\right)\left(\frac{z}{b}\right)^{m}\left(\frac{z^{*}}{b}\right)^{n}
$$

Then one finds by application of an arbitrary operator $\exp \left(-a^{2} \frac{\partial^{2}}{\partial z \partial z^{*}}\right)$ to it

$$
\begin{aligned}
& \exp \left(-a^{2} \frac{\partial^{2}}{\partial z \partial z^{*}}\right) \mathrm{L}_{m, n}\left(\frac{z}{b}, \frac{z^{*}}{b}\right)=\frac{1}{b^{m+n}} \exp \left(-\left(a^{2}+b^{2}\right) \frac{\partial^{2}}{\partial z \partial z^{*}}\right) z^{m} z^{* n} \\
& =\left(\sqrt{\frac{a^{2}+b^{2}}{b^{2}}}\right)^{m+n} \exp \left(-\left(\sqrt{a^{2}+b^{2}}\right)^{2} \frac{\partial^{2}}{\partial z \partial z^{*}}\right)\left(\frac{z}{\sqrt{a^{2}+b^{2}}}\right)^{m}\left(\frac{z^{*}}{\sqrt{a^{2}+b^{2}}}\right)^{n} \\
& =\left(\sqrt{\frac{a^{2}+b^{2}}{b^{2}}}\right)^{m+n} \mathrm{~L}_{m, n}\left(\frac{z}{\sqrt{a^{2}+b^{2}}}, \frac{z^{*}}{\sqrt{a^{2}+b^{2}}}\right)
\end{aligned}
$$

With $a^{2}=-\frac{r(1+r)}{2}, \quad b^{2}=\left(\frac{1+r}{2}\right)^{2} \quad \rightarrow a^{2}+b^{2}=\frac{1-r^{2}}{4}, \quad \frac{a^{2}+b^{2}}{b^{2}}=\frac{1-r}{1+r}$ follows the last part in (B.4).

For the quantum-mechanical operator $A_{r}$ corresponding in the Weyl formalism to the smoothed classical function $A_{r}\left(\alpha, \alpha^{*}\right)$ we obtain

$$
\begin{aligned}
A_{r}= & \frac{2}{\pi(1+r)} \int \frac{\mathrm{i}}{2} \mathrm{~d} \alpha \wedge \mathrm{d} \alpha^{*} A\left(\alpha, \alpha^{*}\right) \exp \left(-\frac{2 \alpha \alpha^{*}}{1+r}\right) \sum_{m=0}^{\infty} \sum_{n=0}^{\infty} \frac{|m\rangle\langle n|}{\sqrt{m ! n !}} \\
& \cdot\left(\sqrt{\frac{1-r}{1+r}}\right)^{m+n} \mathrm{~L}_{m, n}\left(\frac{2 \alpha}{\sqrt{1-r^{2}}}, \frac{2 \alpha^{*}}{\sqrt{1-r^{2}}}\right)
\end{aligned}
$$

For $r=0$ we obtain the Formulas (5.14).

The case $r=1$ has to be considered by a limiting procedure. Setting $r=1-\varepsilon$ we find for $\varepsilon \rightarrow 0$

$$
\begin{aligned}
& \lim _{\varepsilon \rightarrow 0} \exp \left(\frac{1-\varepsilon}{2} \frac{\partial^{2}}{\partial \alpha \partial \alpha^{*}}\right) \exp \left(-a \frac{\partial}{\partial \alpha}-a^{\dagger} \frac{\partial}{\partial \alpha^{*}}\right) \delta\left(\alpha, \alpha^{*}\right) \\
= & \lim _{\varepsilon \rightarrow 0} \frac{\exp \left(-\alpha \alpha^{*}\right)}{\pi} \sum_{m=0}^{\infty} \sum_{n=0}^{\infty} \frac{m\rangle\langle n|}{\sqrt{m ! n !}}\left(\sqrt{\frac{\varepsilon}{2}}\right)^{m+n}\left(\frac{2 \alpha}{\sqrt{2 \varepsilon}}\right)^{m}\left(\frac{2 \alpha^{*}}{\sqrt{2 \varepsilon}}\right)^{n} \\
& \cdot \sum_{j=0}^{\{m, n\}} \frac{(-1)^{j} m ! n !}{j !(m-j) !(n-j) !}\left(\frac{2 \varepsilon}{4 \alpha \alpha^{*}}\right)^{j} \\
= & \frac{\exp \left(-\alpha \alpha^{*}\right)}{\pi} \sum_{m=0}^{\infty} \sum_{n=0}^{\infty} \frac{\alpha^{m} \alpha^{* n}}{\sqrt{m ! n !}}|m\rangle\langle n|=\frac{|\alpha\rangle\langle\alpha|}{\pi}
\end{aligned}
$$

that means 


$$
\begin{aligned}
A_{r \rightarrow 1} & =\frac{1}{\pi} \int \frac{\mathrm{i}}{2} \mathrm{~d} \alpha \wedge \mathrm{d} \alpha^{*} A\left(\alpha, \alpha^{*}\right)|\alpha\rangle\langle\alpha| \\
& =\frac{1}{\pi} \int \frac{\mathrm{i}}{2} \mathrm{~d} \alpha \wedge \mathrm{d} \alpha^{*} A\left(\alpha, \alpha^{*}\right) \exp \left(-\alpha \alpha^{*}\right) \sum_{m=0}^{\infty} \sum_{n=0}^{\infty} \frac{\alpha^{m} \alpha^{* n}}{\sqrt{m ! n !}}|m\rangle\langle n|
\end{aligned}
$$

where $|\alpha\rangle$ are the coherent states which are (over-)complete in the sense of (3.22) for special case $m=n=0$.

For the smoothed special classical functions $A^{(k, l)}\left(\alpha, \alpha^{*}\right)$ in (6.1) that means for

$$
A_{r}^{(k, l)}\left(\alpha, \alpha^{*}\right)=\exp \left(\frac{r}{2} \frac{\partial^{2}}{\partial \alpha \partial \alpha^{*}}\right)\left(\sqrt{\alpha \alpha^{*}}\right)^{k}\left(\sqrt{\frac{\alpha}{\alpha^{*}}}\right)^{l}
$$

starting from (B.7) one obtains in a calculation which is in full analogy to the calculation in (A.9) the following generalization for the number representation of the corresponding operators to the smoothed classical functions $A_{r}^{(k, l)}\left(\alpha, \alpha^{*}\right)$

$$
\begin{aligned}
A_{r}^{(k, l)}= & \sum_{m=0}^{\infty}|m\rangle\langle m+l| \sqrt{m !(m+l) !}\left(\frac{1-r}{1+r}\right)^{m}\left(\frac{2}{1+r}\right)^{-\frac{k-l}{2}} \\
& \cdot \sum_{j=0}^{m} \frac{(-1)^{j}\left(m+\frac{k+l}{2}-j\right) !}{j !(m-j) !(m+l-j) !}\left(\frac{2}{1-r}\right)^{m-j}
\end{aligned}
$$

which, clearly, for $r=0$ become identical with (A.9).

The general Formulas (B.11) can be written in more compact form but in alternative ways using the Jacobi polynomials $\mathrm{P}_{n}^{(\alpha, \beta)}(u)$. We make this in the main text from Formulas (8.2) on and discuss it there.

\section{Appendix C}

Sequences of relations for symmetrically ordered powers $\mathcal{S}\left\{a^{\dagger k} a^{k}\right\}$ of annihilation and creation operator

We give in this Appendix for practical purpose a few initial terms for the relations of the symmetrically ordered operators $\mathcal{S}\left\{a^{\dagger k} a^{k}\right\}$ to representation in normal ordering and to expansion in powers of the number operator $N$.

Using relation (12.3) one obtains the following relations of $\mathcal{S}\left\{a^{\dagger k} a^{k}\right\}$ to normally ordered powers of the annihilation and creation operators up to $k=6$ :

Sequence 1: Representations of symmetrically ordered operators $\mathcal{S}\left\{a^{\dagger k} a^{k}\right\}$ in normal ordering

$$
\begin{aligned}
& \mathcal{S}\left\{a^{\dagger 0} a^{0}\right\}=I \\
& \mathcal{S}\left\{a^{\dagger 1} a^{1}\right\}=a^{\dagger} a+\frac{1}{2} I \\
& \mathcal{S}\left\{a^{\dagger 2} a^{2}\right\}=a^{\dagger 2} a^{2}+2 a^{\dagger} a+\frac{1}{2} I \\
& \mathcal{S}\left\{a^{\dagger 3} a^{3}\right\}=a^{\dagger 3} a^{3}+\frac{9}{2} a^{\dagger 2} a^{2}+\frac{9}{2} a^{\dagger} a+\frac{3}{4} I
\end{aligned}
$$




$$
\begin{aligned}
\mathcal{S}\left\{a^{\dagger 4} a^{4}\right\}= & a^{\dagger 4} a^{4}+8 a^{\dagger 3} a^{3}+18 a^{\dagger 2} a^{2}+12 a^{\dagger} a+\frac{3}{2} I \\
\mathcal{S}\left\{a^{\dagger 5} a^{5}\right\}= & a^{\dagger 5} a^{5}+\frac{25}{2} a^{\dagger 4} a^{4}+50 a^{\dagger 3} a^{3}+75 a^{\dagger 2} a^{2}+\frac{75}{2} a^{\dagger} a+\frac{15}{4} I \\
\mathcal{S}\left\{a^{\dagger 6} a^{6}\right\}= & a^{\dagger 6} a^{6}+18 a^{\dagger 5} a^{5}+\frac{225}{2} a^{\dagger 4} a^{4}+300 a^{\dagger 3} a^{3} \\
& +\frac{675}{2} a^{\dagger 2} a^{2}+135 a^{\dagger} a+\frac{45}{4} I
\end{aligned}
$$

According to the analogous structure of (12.3) and (12.4) the inversion of the relations in (C.1) is very simple and is connected with changing signs of sum terms.

Using for arbitrary real variable $x$ the formula

$$
(x)_{k} \equiv \frac{x !}{(x-k) !}=\sum_{l=0}^{k} \mathrm{~s}(k, l) x^{l}
$$

where $s(k, l)$ denotes the Stirling numbers of first kind one may prove the following relation between from $a^{\dagger k} a^{k}$ to powers of $N=a^{\dagger} a$ and vice versa

$$
a^{\dagger k} a^{k}=\frac{N !}{(N-k I) !}=\sum_{l=0}^{k} \mathrm{~s}(k, l) N^{l} \Leftrightarrow N^{k}=\sum_{l=0}^{k} \mathrm{~s}(k, l) a^{\dagger l} a^{l}
$$

where $S(k, l)$ denotes the Stirling numbers of second kind and we obtain

$$
\begin{aligned}
\left(\sum_{l=0}^{k} \mathrm{~s}(k, l) \mathrm{S}(l, m)=\delta_{k, m}\right) & \\
\mathcal{S}\left\{a^{\dagger k} a^{k}\right\} & =\sum_{j=0}^{k} \frac{k !^{2}}{j !(k-j) !^{2}}\left(\frac{1}{2}\right)^{j} a^{\dagger k-j} a^{k-j} \\
& =\sum_{l=0}^{k}\left(\sum_{j=0}^{k} \frac{k !^{2}}{2^{j} j !(k-j) !^{2}} \mathrm{~s}(k-j, k-l)\right) N^{k-l}
\end{aligned}
$$

The following table gives a few initial members of this relation.

Sequence 2: Representations of symmetrically ordered operators $\mathcal{S}\left\{a^{\dagger k} a^{k}\right\}$ in powers of $N$

$$
\begin{aligned}
\mathcal{S}\left\{a^{\dagger 0} a^{0}\right\} & =I \\
\mathcal{S}\left\{a^{\dagger 1} a^{1}\right\} & =N+\frac{1}{2} I \\
\mathcal{S}\left\{a^{\dagger 2} a^{2}\right\} & =N^{2}+N+\frac{1}{2} I=\left(N+\frac{1}{2} I\right)^{2}+\frac{1}{4} I \\
\mathcal{S}\left\{a^{\dagger 3} a^{3}\right\} & =N^{3}+\frac{3}{2} N^{2}+2 N+\frac{3}{4} I=\left(N+\frac{1}{2} I\right)^{3}+\frac{5}{4}\left(N+\frac{1}{2} I\right) \\
\mathcal{S}\left\{a^{\dagger 4} a^{4}\right\} & =N^{4}+2 N^{3}+5 N^{2}+4 N+\frac{3}{2} I=\left(N+\frac{1}{2} I\right)^{4}+\frac{7}{2}\left(N+\frac{1}{2} I\right)^{2}+\frac{9}{16} I \\
\mathcal{S}\left\{a^{\dagger 5} a^{5}\right\} & =N^{5}+\frac{5}{2} N^{4}+10 N^{3}+\frac{25}{2} N^{2}+\frac{23}{2} N+\frac{15}{4} I \\
& =\left(N+\frac{1}{2} I\right)^{5}+\frac{15}{2}\left(N+\frac{1}{2} I\right)^{3}+\frac{89}{16}\left(N+\frac{1}{2} I\right)
\end{aligned}
$$




$$
\begin{aligned}
\mathcal{S}\left\{a^{\dagger 6} a^{6}\right\} & =N^{6}+3 N^{5}+\frac{35}{2} N^{4}+30 N^{3}+49 N^{2}+\frac{69}{2} N+\frac{45}{4} I \\
& =\left(N+\frac{1}{2} I\right)^{6}+\frac{55}{4}\left(N+\frac{1}{2} I\right)^{4}+\frac{439}{16}\left(N+\frac{1}{2} I\right)^{2}+\frac{225}{64} I
\end{aligned}
$$

Since the coefficients 89 and 439 are high prime numbers in comparison to $k=5$ and $k=6$ it seems to be not easy to find a simple closed formula for the representation of the left-hand sides in powers of $N+\frac{1}{2} I=\mathcal{S}\left\{a a^{\dagger}\right\}=\mathcal{S}\left\{a^{\dagger} a\right\}$. Besides a triple sum resulting from (C.4) by application of the binomial formula for $N^{k-l}=\left(\left(N+\frac{1}{2} I\right)-\frac{1}{2} I\right)^{k-l}$ we did not find up to now an approach leading to a more compact relation, for example, only by a double sum. The calculated coefficients at the powers of $\left(N+\frac{1}{2} I\right)$ in (C.5) are non-negative and are non-vanishing in decreasing steps of 2 beginning from the highest power of $k$. We checked this by computer up to "sufficiently" high $k$ but could not prove this up to now. 